\title{
Pd-Catalyzed Oxidative Annulation of Aryl Ethers with Alkynes: Synthesis of Functionalized Spirocycles and \\ Naphthalenes
}

Shiyong Peng, ${ }^{\dagger, \dagger, \#}$ Zhonghao Sun,${ }^{\dagger, \#}$ Huijuan Zhu, ${ }^{\dagger}$ Nuan Chen,${ }^{\dagger}$ Xiaobo Sun, ${ }^{\dagger}$ Xiaojie Gong, ${ }^{, \S}$ Jian Wang, $* *$ and Lei Wang $*, \dagger$

†nstitute of Medicinal Plant Development, Chinese Academy of Medical Science and Peking Union

Medical College, Beijing, 100193, P. R. China

${ }^{\ddagger}$ School of Biotechnology and Health Sciences, Wuyi University, Jiangmen 529020, P. R. China

${ }^{\S}$ College of Life Science, Dalian Minzu University, Dalian 116600, P. R. China

E-mail: wangjian2012@tsinghua.edu.cn; gxjclr@163.com; 1wang@implad.ac.cn

\section{Supporting Information}

\section{CONTENTS}

1. General information ....................................................

2. Preparation of starting materials ..................................... 2

3. Preparation and characterization of compounds $3 \ldots \ldots \ldots \ldots \ldots \ldots \ldots \ldots$

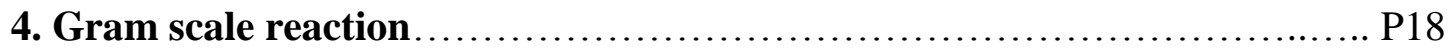

5. Intermolecular competition experiments..............................P19

6. KIE experiments...................................................... 21

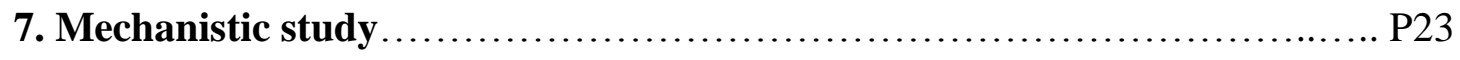

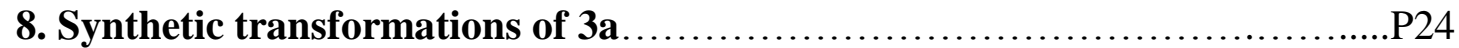

9. Preparation and characterization of compounds $5 \ldots \ldots \ldots \ldots \ldots \ldots \ldots \ldots$. 25

10. Proposed mechamism for the formation of compounds $5 \ldots \ldots \ldots \ldots \ldots \ldots . . . .230$

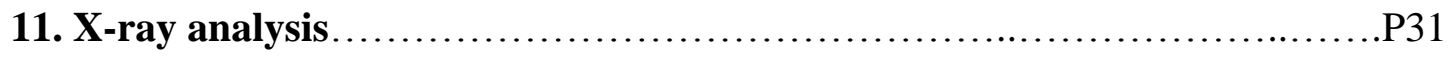

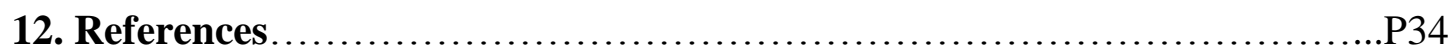

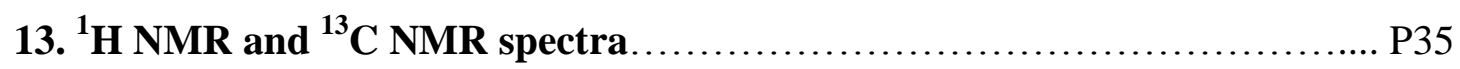




\section{General information.}

Chemicals and solvents were purchased from commercial suppliers and used as received. ${ }^{1} \mathrm{H}$ and ${ }^{13} \mathrm{C}$ NMR spectra were recorded on a Bruker AMX500 (500 MHz) spectrometer. Chemical shifts were reported in parts per million (ppm), and the residual solvent peak was used as an internal reference. ${ }^{[1]}$ Multiplicity was indicated as follows: $\mathrm{s}$ (singlet), $\mathrm{d}$ (doublet), $\mathrm{t}$ (triplet), $\mathrm{q}$ (quartet), m (multiplet), dd (doublet of doublet), bs (broad singlet). Coupling constants were reported in Hertz (Hz). Low resolution mass spectra were obtained on a Finnigan/MAT LCQ spectrometer in ESI mode, and a Finnigan/MAT 95XL-T mass spectrometer in EI mode. All high resolution mass spectra were obtained on a Finnigan/MAT 95XL-T mass spectrometer. For thin layer chromatography (TLC), Merck pre-coated TLC plates (Merck 60 F254) were used, and compounds were visualized with a UV light at $254 \mathrm{~nm}$. Flash chromatography separations were performed on Merck 60 (0.040-0.063 mm) mesh silica gel.

\section{Preparation of starting materials.}

1a-n were purchased from commercial suppliers and used as received. $\mathbf{2 a}, \mathbf{2 o}$ and $\mathbf{2 p}$ were purchased from commercial suppliers and used as received. The other alkynes 2b-n were synthesized according to related literatures with some modification. ${ }^{[2]}$<smiles>COc1ccccc1</smiles>

$1 \mathrm{a}$<smiles>COc1cccc(F)c1</smiles>

$1 e$<smiles>COc1cc(C)cc(C)c1</smiles>

$1 \mathbf{j}$<smiles>CCOc1ccccc1</smiles>

1a'<smiles>COc1cccc(C)c1C</smiles>

$1 f$<smiles>COc1cc(F)cc(OC)c1</smiles>

$1 k$<smiles>COc1ccccc1C</smiles>

$1 b$<smiles>COc1c(C)cccc1C</smiles>

$1 g$<smiles>COc1cccc2ccccc12</smiles>

11<smiles>COc1ccccc1F</smiles>

1c<smiles>COc1c(Cl)cccc1Cl</smiles>

1h<smiles>COc1ccccc1OC</smiles>

$1 \mathrm{~m}$<smiles>COc1ccccc1Br</smiles>

1d<smiles>COc1cc(C)ccc1C</smiles>

1 i<smiles>COc1cccc(OC)c1OC</smiles>

1n 


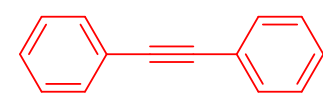

$2 \mathbf{a}$

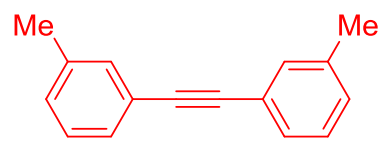

2d

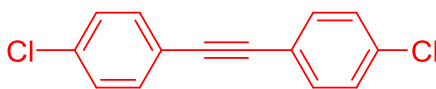

$2 \mathrm{~g}$

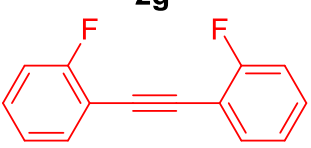

2j

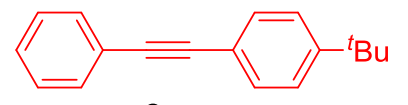

$2 \mathrm{~m}$

$\mathrm{Et} \rightleftharpoons \mathrm{Et}$

$2 p$

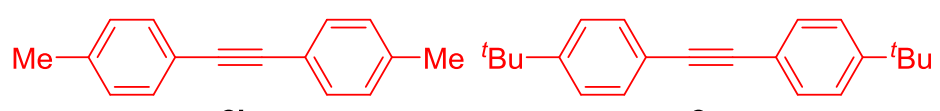

2c

$2 b$
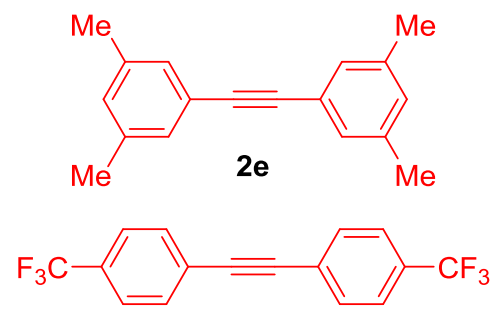

$2 h$
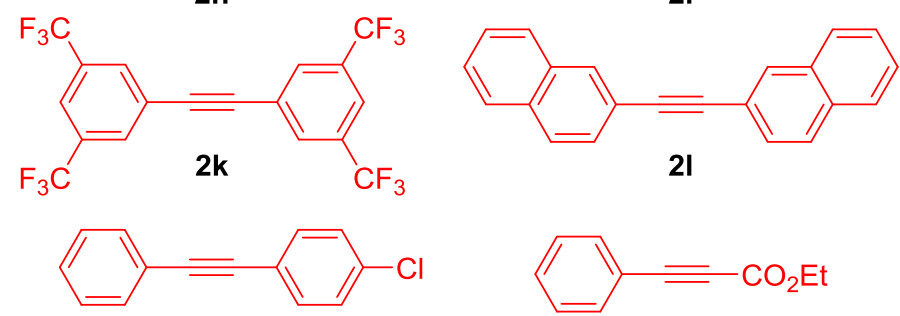

$2 n$
21

$2 f$

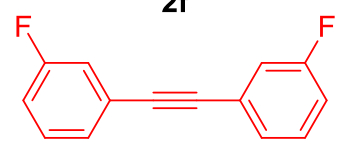

$2 \mathrm{i}$
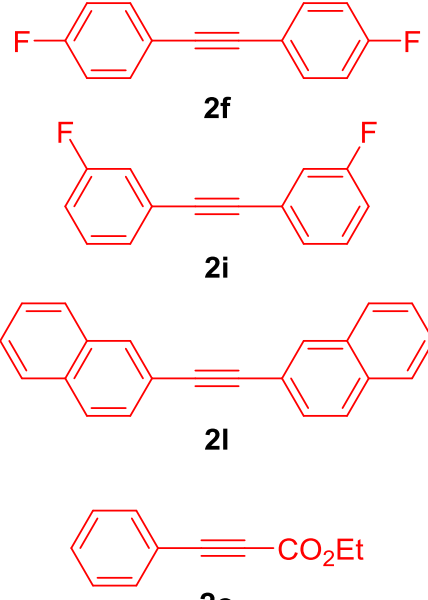

\section{General procedure for the preparation of compounds 3}<smiles>[R]C#C[Fe]</smiles><smiles>[R]C1=C([R])C2(C=CC(=O)C=[R1])C([R])=C([R])C12[R]</smiles>

To a solution of alkyne $2(0.4 \mathrm{mmol})$ in anisole $\mathbf{1}(2.0 \mathrm{ml})$, palladium (II) acetate (10 mol \%), HOAc $(0.4 \mathrm{mmol})$ and potassium persulfate $(0.8 \mathrm{mmol})$ were added. The reaction mixture was stirred at $80{ }^{\circ} \mathrm{C}$ in an oil bath under $\mathrm{N}_{2}$ until 2 was gone (monitored by TLC). Work-up: The reaction mixture was cooled down to room temperature. Solvent was removed under ScanVac CoolSafe Freeze Dryer. The crude product was pre-adsorbed on silica gel and purified by flash chromatography (silica gel, EtOAc/hexanes) to afford pure product 3.<smiles>O=C1C=CC2(C=C1)C(c1ccccc1)=C(c1ccccc1)C(c1ccccc1)=C2c1ccccc1</smiles>

1,2,3,4-Tetraphenylspiro[4.5]deca-1,3,6,9-tetraen-8-one (3a): ${ }^{[3]}$ The title compound 
was prepared via the general procedure from anisole $1 \mathbf{1 a}(2.0 \mathrm{~mL})$ and 1,2-diphenylethyne $\mathbf{2 a}(0.4 \mathrm{mmol}, 71.3 \mathrm{mg})$. After purification by silica gel column chromatography (hexanes/EtOAc $=20: 1)$, the product was isolated in $81 \%$ yield $(72.7$ mg) as a a light yellow solid. ${ }^{1} \mathbf{H}$ NMR (500 MHz, $\left.\mathbf{C D C l}_{3}\right): \delta 7.19-7.09$ (m, 16H), $6.93(\mathrm{~d}, J=1.5 \mathrm{~Hz}, 4 \mathrm{H}), 6.86(\mathrm{~d}, J=9.5 \mathrm{~Hz}, 2 \mathrm{H}), 6.45(\mathrm{~d}, J=9.5 \mathrm{~Hz}, 2 \mathrm{H}) .{ }^{13} \mathbf{C} \mathbf{N M R}$ (125 MHz, $\left.\mathbf{C D C l}_{3}\right): \delta 186.2,147.9,147.6,141.2,134.7,134.5,131.8,129.9,129.3$, 128.0, 127.9, 127.5, 127.3, 77.3, 77.0, 76.8, 66.2. HRMS (ESI): calcd for $\mathrm{C}_{34} \mathrm{H}_{24} \mathrm{ONa}$ $[\mathrm{M}+\mathrm{Na}]^{+} 471.1719$, found 471.1726 .<smiles>CC1=CC2(C=CC1=O)C(c1ccccc1)=C(c1ccccc1)C(c1ccccc1)=C2c1ccccc1</smiles>

7-Methyl-1,2,3,4-tetraphenylspiro[4.5]deca-1,3,6,9-tetraen-8-one (3b): The title compound was prepared via the general procedure from 1-methoxy-2-methylbenzene $1 \mathbf{b}(2.0 \mathrm{~mL})$ and 1,2-diphenylethyne 2a $(0.4 \mathrm{mmol}, 71.3 \mathrm{mg})$. After purification by silica gel column chromatography (hexanes/EtOAc $=20: 1$ ), the product was isolated in $87 \%$ yield $(80.5 \mathrm{mg})$ as a light yellow solid. IR $(\mathrm{KBr}) / \mathrm{cm}^{-1}: 3028,2920,1656$, 1632, 1488, 1442, 697. ${ }^{1} \mathbf{H}$ NMR (500 MHz, $\left.\mathbf{C D C l}_{3}\right): \delta$ 7.18-7.14 (m, 10H), 7.12-7.05 (m, 3H), 6.93-6.91 (m, 4H), 6.83-6.82 (m, 3H), 6.80 (d, J = 2.5 Hz, 1H), $6.80(\mathrm{~d}, J=1.5 \mathrm{~Hz}, 1 \mathrm{H}), 6.45(\mathrm{~d}, J=9.5 \mathrm{~Hz}, 1 \mathrm{H}), 1.92$ (s, 3H). ${ }^{13} \mathbf{C}$ NMR (125 MHz, $\left.\mathbf{C D C l}_{3}\right): \delta 187.2,148.0,147.5,143.1,142.0,141.1,140.8,138.8,135.3,135.1,131.9$, 130.4, 129.8, 129.7, 128.32, 128.29, 127.8, 127.6, 127.0, 125.6, 77.3, 77.0, 76.8, 66.2. HRMS (ESI): calcd for $\mathrm{C}_{35} \mathrm{H}_{26} \mathrm{ONa}[\mathrm{M}+\mathrm{Na}]^{+} 485.1881$, found 485.1889 .<smiles>O=C1C=CC2(C=C1F)C(c1ccccc1)=C(c1ccccc1)C(c1ccccc1)=C2c1ccccc1</smiles>

7-Fluoro-1,2,3,4-tetraphenylspiro[4.5]deca-1,3,6,9-tetraen-8-one (3c): The title compound was prepared via the general procedure from 1-fluoro-2-methoxybenzene 1c $(2.0 \mathrm{~mL})$ and 1,2-diphenylethyne $2 \mathbf{a}(0.4 \mathrm{mmol}, 71.3 \mathrm{mg})$. After purification by 
silica gel column chromatography (hexanes/EtOAc $=20: 1$ ), the product was isolated in $75 \%$ yield $(70.0 \mathrm{mg})$ as a light yellow solid. IR $(\mathrm{KBr}) / \mathrm{cm}^{-1}: 3055,2932,1675$, 1653, 1514, 1442, 1281, 697. ${ }^{1} \mathbf{H}$ NMR (500 MHz, $\left.\mathbf{C D C l}_{3}\right): \delta 7.21-7.10$ (m, 12H), 7.09-7.05 (m, 4H), 6.94-6.89 (m, 4H), $6.83(\mathrm{dd}, J=9.7,2.4 \mathrm{~Hz}, 1 \mathrm{H}), 6.48(\mathrm{dd}, J=$ 9.7, $6.6 \mathrm{~Hz}, 1 \mathrm{H}), 6.43(\mathrm{dd}, J=12.0,2.5 \mathrm{~Hz}, 1 \mathrm{H}) .{ }^{13} \mathbf{C}$ NMR (125 MHz, $\left.\mathbf{C D C l}_{3}\right): \delta$ $178.7(\mathrm{~d}, J=21.2 \mathrm{~Hz}), 156.4(\mathrm{~d}, J=264.6 \mathrm{~Hz}), 148.9(\mathrm{~d}, J=1.5 \mathrm{~Hz}), 147.5,140.5$, 134.3, 134.1, 131.5 (d, $J=4.2 \mathrm{~Hz}), 130.0,129.3,128.2,127.9,127.7,127.4,123.2$ (d, $J=14.6 \mathrm{~Hz}), 77.3,77.0,76.8,66.4(\mathrm{~d}, J=5.6 \mathrm{~Hz})$. HRMS (ESI): calcd for $\mathrm{C}_{34} \mathrm{H}_{23} \mathrm{OFNa}[\mathrm{M}+\mathrm{Na}]^{+} 489.1631$, found 489.1645 .<smiles>O=C1C=CC2(C=C1Br)C(c1ccccc1)=C(c1ccccc1)C(c1ccccc1)=C2c1ccccc1</smiles>

7-Bromo-1,2,3,4-tetraphenylspiro[4.5]deca-1,3,6,9-tetraen-8-one (3d): The title compound was prepared via the general procedure from 1-bromo-2-methoxybenzene 1d $(2.0 \mathrm{~mL})$ and 1,2-diphenylethyne $2 \mathbf{a}(0.4 \mathrm{mmol}, 71.3 \mathrm{mg})$. After purification by silica gel column chromatography (hexanes/EtOAc $=20: 1)$, the product was isolated in $70 \%$ yield $(73.8 \mathrm{mg})$ as a light yellow solid. ${ }^{1} \mathbf{H}$ NMR (500 $\left.\mathbf{M H z}, \mathbf{C D C l}_{3}\right)$ : $\delta$ 7.32-7.30 (m, 1H), 7.23-7.06 (m, 16H), 6.94-6.88 (m, 5H), 6.56 (d, J=9.5 Hz, 1H). ${ }^{13}$ C NMR (125 MHz, $\left.\mathbf{C D C l}_{3}\right): \delta 179.3,148.4,148.1,140.5,134.7,134.4,130.8$, 130.3, 129.7, 128.6, 128.4, 128.2, 127.9, 126.9, 77.3, 77.0, 76.8, 69.4. HRMS (ESI): calcd for $\mathrm{C}_{34} \mathrm{H}_{23} \mathrm{OBrNa}[\mathrm{M}+\mathrm{Na}]^{+}$549.0830, found 549.0839.

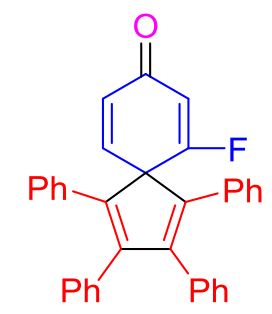

6-Fluoro-1,2,3,4-tetraphenylspiro[4.5]deca-1,3,6,9-tetraen-8-one (3e): The title compound was prepared via the general procedure from 1-fluoro-3-methoxybenzene 1e $(2.0 \mathrm{~mL})$ and 1,2-diphenylethyne $\mathbf{2 a}(0.4 \mathrm{mmol}, 71.3 \mathrm{mg})$. After purification by silica gel column chromatography (hexanes/EtOAc $=20: 1$ ), the product was isolated 
in $70 \%$ yield $(65.3 \mathrm{mg})$ as a light yellow solid. ${ }^{\mathbf{1}} \mathbf{H}$ NMR (500 $\left.\mathbf{M H z}, \mathbf{C D C l}_{3}\right)$ : $\delta$ 7.19-7.04 (m, 14H), 6.90-6.83 (m, 6H), $6.64(\mathrm{~d}, J=9.5 \mathrm{~Hz}, 1 \mathrm{H}), 6.09$ (d, $J=1.5 \mathrm{~Hz}$, $1 \mathrm{H}), 6.06(\mathrm{~d}, J=1.5 \mathrm{~Hz}, 1 \mathrm{H}) .{ }^{\mathbf{1 3}} \mathbf{C}$ NMR (125 MHz, $\left.\mathbf{C D C l}_{3}\right): \delta 189.0(\mathrm{~d}, J=15.5 \mathrm{~Hz})$, $174.3(\mathrm{~d}, J=283.3 \mathrm{~Hz}), 149.4,144.4,144.4,141.1,140.8,140.3$, 134.7, 131.9, 131.0, 130.4, 129.6, 128.7, 128.33, 128.26, 127.9, 127.0, 125.7, 113.8 (d, $J=10.9 \mathrm{~Hz}), 77.3$, 77.0, 76.8, $67.3(\mathrm{~d}, J=24.6 \mathrm{~Hz})$. HRMS (ESI): calcd for $\mathrm{C}_{34} \mathrm{H}_{23} \mathrm{OFNa}[\mathrm{M}+\mathrm{Na}]^{+}$ 489.1631, found 489.1632 .<smiles>CC1=C(c2ccccc2)C2(C=CC1=O)C(c1ccccc1)=C(C)C(c1ccccc1)=C2c1ccccc1</smiles>

6,7-Dimethyl-1,2,3,4-tetraphenylspiro[4.5]deca-1,3,6,9-tetraen-8-one $\quad(3 f)$ : The title compound was prepared via the general procedure from 1-methoxy-2,3-dimethylbenzene $\mathbf{1 f}(2.0 \mathrm{~mL})$ and 1,2-diphenylethyne $\mathbf{2 a}(0.4 \mathrm{mmol}$, $71.3 \mathrm{mg}$ ). After purification by silica gel column chromatography (hexanes/EtOAc $=$ 20:1), the product was isolated in $81 \%$ yield $(77.2 \mathrm{mg})$ as a pale yellow solid. IR $(\mathrm{KBr}) / \mathrm{cm}^{-1}: 3026,2921,1656,1633,1488,1441,696 .{ }^{1} \mathbf{H}$ NMR (500 MHz, CDCl $\left.\mathbf{~}_{3}\right)$ : $\delta$ 7.16-7.04 (m, 12H), 6.98-6.90 (m, 8H), $6.72(\mathrm{~d}, J=9.5 \mathrm{~Hz}, 1 \mathrm{H}), 6.46(\mathrm{~d}, J=9.5$ $\mathrm{Hz}, 1 \mathrm{H}), 1.86$ (s, 3H), 1.83 (s, 3H). ${ }^{\mathbf{1 3}} \mathbf{C}$ NMR (125 MHz, $\left.\mathbf{C D C l}_{3}\right): \delta$ 186.8, 149.5, $148.6,147.7,143.0,136.4,135.4,134.8,130.9$, 130.4, 129.4, 128.4, 127.8, 127.7, 77.3, 77.0, 76.8, 69.6, 16.5, 11.6. HRMS (ESI): calcd for $\mathrm{C}_{36} \mathrm{H}_{28} \mathrm{ONa}[\mathrm{M}+\mathrm{Na}]^{+}$ 499.2038, found 499.2057.<smiles>CC1=CC2(C=C(C)C1=O)C(c1ccccc1)=C(c1ccccc1)C(c1ccccc1)=C2c1ccccc1</smiles>

7,9-Dimethyl-1,2,3,4-tetraphenylspiro[4.5]deca-1,3,6,9-tetraen-8-one $(3 \mathrm{~g}):$ The title compound was prepared via the general procedure from 2-methoxy-1,3-dimethylbenzene $\mathbf{1 g}(2.0 \mathrm{~mL})$ and 1,2-diphenylethyne $\mathbf{2 a}(0.4 \mathrm{mmol}$, $71.3 \mathrm{mg}$ ). After purification by silica gel column chromatography (hexanes/EtOAc $=$ 
20:1), the product was isolated in $83 \%$ yield $(79.1 \mathrm{mg})$ as a light yellow solid. IR $(\mathrm{KBr}) / \mathrm{cm}^{-1}:$ 3029, 2919, 1661, 1631, 1441, 753. ${ }^{\mathbf{1}} \mathbf{H}$ NMR (500 MHz, $\left.\mathbf{C D C l}_{3}\right): \delta$ 7.20-7.08 (m, 12H), 7.06-7.00 (m, 4H), 6.95-6.89 (m, 4H), $6.60(\mathrm{~s}, 2 \mathrm{H}), 1.90(\mathrm{~s}, 6 \mathrm{H})$. ${ }^{13}$ C NMR (125 MHz, CDCl 3 ): $\delta$ 187.3, 146.5, 142.3, 141.9, 137.8, 135.0, 134.8, 129.9, 129.3, 127.80, 127.77, 127.2, 127.0, 77.3, 77.0, 76.8, 66.1, 16.3. HRMS (ESI): calcd for $\mathrm{C}_{36} \mathrm{H}_{28} \mathrm{ONa}[\mathrm{M}+\mathrm{Na}]^{+}$499.2038, found 499.2050.<smiles>O=C1C(Cl)=CC2(C=C1Cl)C(c1ccccc1)=C(c1ccccc1)C(c1ccccc1)=C2c1ccccc1</smiles>

7,9-Dichloro-1,2,3,4-tetraphenylspiro [4.5] deca-1,3,6,9-tetraen-8-one (3h): The title compound was prepared via the general procedure from 1,3-dichloro-2-methoxybenzene $\mathbf{1 h}(2.0 \mathrm{~mL})$ and 1,2-diphenylethyne $\mathbf{2 a}(0.4 \mathrm{mmol}$, $71.3 \mathrm{mg})$. After purification by silica gel column chromatography $($ hexanes/EtOAc $=$ 20:1), the product was isolated in $65 \%$ yield $(67.3 \mathrm{mg})$ as a light yellow solid. ${ }^{1} \mathbf{H}$ NMR (500 MHz, CDCl 3 ): $\delta$ 7.90-7.87 (m, 4H), 7.48-7.43 (m, 2H), 7.38-7.30 (m, 4H), 7.24-7.22 (m, 11H), $6.88(\mathrm{~d}, J=1.5 \mathrm{~Hz}, 1 \mathrm{H}) .{ }^{13} \mathbf{C}$ NMR (125 MHz, $\left.\mathbf{C D C l}_{3}\right): \delta$ $197.4,145.1,136.9,135.7,133.5,131.9,130.5,130.3,129.1,128.9,128.8,127.0$, 87.0, 77.3, 77.0, 76.8. HRMS (EI): calcd for $\mathrm{C}_{34} \mathrm{H}_{22} \mathrm{OCl}_{2}[\mathrm{M}]^{+}$516.1048, found 516.1050 .

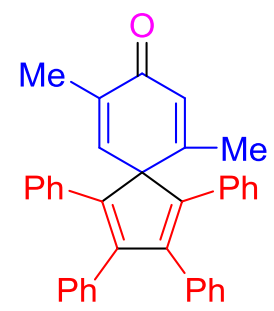

6,9-Dimethyl-1,2,3,4-tetraphenylspiro[4.5]deca-1,3,6,9-tetraen-8-one (3i): The title compound was prepared via the general procedure from 2-methoxy-1,4-dimethylbenzene $\mathbf{1 i}(2.0 \mathrm{~mL})$ and 1,2-diphenylethyne $\mathbf{2 a}(0.4 \mathrm{mmol}$, $71.3 \mathrm{mg})$. After purification by silica gel column chromatography $($ hexanes/EtOAc $=$ 20:1), the product was isolated in $85 \%$ yield $(81.0 \mathrm{mg})$ as a yellow solid. IR $(\mathrm{KBr}) /$ $\mathrm{cm}^{-1}:$ 3025, 2922, 1662, 1635, 1487, 1441, 697. ${ }^{\mathbf{1}} \mathbf{H}$ NMR (500 MHz, $\left.\mathbf{C D C l}_{3}\right): \delta$ 
7.16-7.06 (m, 12H), 6.99-6.90 (m, 8H), $6.53(\mathrm{~d}, J=1.5 \mathrm{~Hz}, 1 \mathrm{H}), 6.32(\mathrm{~d}, J=1.5 \mathrm{~Hz}$, 1H), 1.94 (s, 6H). ${ }^{13} \mathbf{C}$ NMR (125 MHz, $\left.\mathbf{C D C l}_{3}\right): \delta$ 187.9, 156.6, 148.4, 143.5, 142.9, $137.9,135.4,134.7,131.3,130.3,129.4,128.39,128.38,127.8,127.7,77.3,77.0$, 76.8, 69.3, 19.1, 16.1. HRMS (ESI): calcd for $\mathrm{C}_{36} \mathrm{H}_{28} \mathrm{ONa}[\mathrm{M}+\mathrm{Na}]^{+} 499.2038$, found 499.2056.

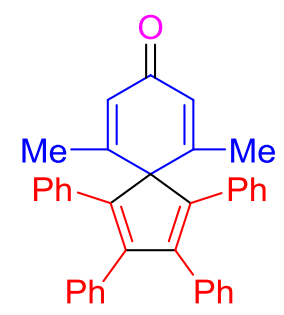

6,10-Dimethyl-1,2,3,4-tetraphenylspiro[4.5]deca-1,3,6,9-tetraen-8-one $(3 \mathbf{j}):^{[3]}$ The title compound was prepared via the general procedure from 1-methoxy-3,5-dimethylbenzene $\mathbf{1 j}(2.0 \mathrm{~mL})$ and 1,2-diphenylethyne $\mathbf{2 a}(0.4 \mathrm{mmol}$, $71.3 \mathrm{mg}$ ). After purification by silica gel column chromatography (hexanes/EtOAc = 20:1), the product was isolated in $60 \%$ yield $(57.2 \mathrm{mg})$ as a yellow solid. ${ }^{1} \mathbf{H}$ NMR (500 MHz, $\left.\mathbf{C D C l}_{3}\right): \delta$ 7.23-7.07 (m, 12H), 7.02-6.96 (m, 4H), 6.96-6.88 (m, 4H), 6.35 (s, 2H), 1.97 (s, 6H). ${ }^{13}$ C NMR (125 MHz, $\left.\mathbf{C D C l}_{3}\right): \delta$ 186.6, 156.7, 149.2, 143.2, $134.9,133.8,130.8,129.8,128.7,128.13,128.06,127.5,127.4,77.3,77.0,76.8,71.2$, 18.4. HRMS (ESI): calcd for $\mathrm{C}_{36} \mathrm{H}_{28} \mathrm{ONa}[\mathrm{M}+\mathrm{Na}]^{+}$499.2038, found 499.2054.

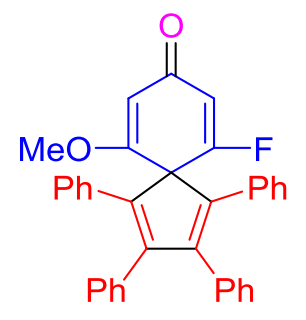

6-Fluoro-10-methoxy-1,2,3,4-tetraphenylspiro[4.5]deca-1,3,6,9-tetraen-8-one (3k): The title compound was prepared via the general procedure from 1-fluoro-3,5-dimethoxybenzene $\mathbf{1 k}(2.0 \mathrm{~mL})$ and 1,2-diphenylethyne $\mathbf{2 a}(0.4 \mathrm{mmol}$, $71.3 \mathrm{mg}$ ). After purification by silica gel column chromatography (hexanes/EtOAc = $20: 1)$, the product was isolated in $67 \%$ yield $(66.5 \mathrm{mg})$ as a yellow solid. IR (KBr) / $\mathrm{cm}^{-1}:$ 3051, 2941, 1674, 1638, 1603, 1441, 1228, 697. ${ }^{\mathbf{1}}$ H NMR (500 MHz, CDCl 3 ): א 7.22-7.14 (m, 8H), 7.14-7.08 (m, 4H), 7.06-6.99 (m, 4H), 6.97-6.92 (m, 4H), 6.02 $(\mathrm{d}, J=12.7 \mathrm{~Hz}, 1 \mathrm{H}), 5.64(\mathrm{~s}, 1 \mathrm{H}), 3.71(\mathrm{~s}, 3 \mathrm{H}) .{ }^{13} \mathbf{C}$ NMR (125 MHz, CDCl $): \delta$ 
$188.2(\mathrm{~d}, J=17.3 \mathrm{~Hz}), 171.0(\mathrm{~d}, J=276.9 \mathrm{~Hz}), 149.7,141.1,141.0,140.8,134.8$, $134.2,131.9,130.4,129.4,128.6,128.2,128.1,127.8,127.0,125.6,111.9$ (d, $J=11.8$ Hz), 105.2, 77.3, 77.0, 76.8, $69.1(\mathrm{~d}, J=25.5 \mathrm{~Hz}), 56.9$. HRMS (ESI): calcd for $\mathrm{C}_{35} \mathrm{H}_{25} \mathrm{O}_{2} \mathrm{FNa}[\mathrm{M}+\mathrm{Na}]^{+}$519.1736, found 519.1739.

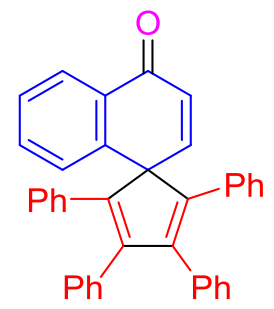

\section{2,3,4,5-Tetraphenyl-4' H-spiro[cyclopenta [2,4] diene-1,1'-naphthalen]-4'-one (31):}

The title compound was prepared via the general procedure from 1-methoxynaphthalene $\mathbf{1 l}(2.0 \mathrm{~mL})$ and 1,2-diphenylethyne $\mathbf{2 a}(0.4 \mathrm{mmol}, 71.3 \mathrm{mg})$. After purification by silica gel column chromatography (hexanes/EtOAc $=20: 1$ ), the product was isolated in $68 \%$ yield $(67.8 \mathrm{mg})$ as a yellow solid. IR $(\mathrm{KBr}) / \mathrm{cm}^{-1}: 3023$, 2926, 1663, 1633, 1594, 1441, 1299, 696. ${ }^{\mathbf{H}} \mathbf{H}$ NMR (500 MHz, $\left.\mathbf{C D C l}_{3}\right): \delta 8.17-8.08$ (m, 1H), 7.56-7.49 (m, 1H), 7.45-7.34 (m, 2H), 7.23-7.10 (m, 6H), 7.07-6.94 (m, $10 \mathrm{H}), 6.92(\mathrm{~d}, J=9.9 \mathrm{~Hz}, 1 \mathrm{H}), 6.80-6.70(\mathrm{~m}, 4 \mathrm{H}), 6.61(\mathrm{~d}, J=9.9 \mathrm{~Hz}, 1 \mathrm{H}) .{ }^{13} \mathbf{C}$ NMR (125 MHz, $\left.\mathbf{C D C l}_{3}\right): \delta 185.1,148.6,146.8,145.7,140.0,134.9,134.4,133.2,132.8$, 130.6, 130.0, 129.3, 127.9, 127.78, 127.76, 127.2, 127.1, 126.4, 77.3, 77.0, 76.8, 66.3. HRMS (ESI): calcd for $\mathrm{C}_{38} \mathrm{H}_{26} \mathrm{ONa}[\mathrm{M}+\mathrm{Na}]^{+}$521.1881, found 521.1890.

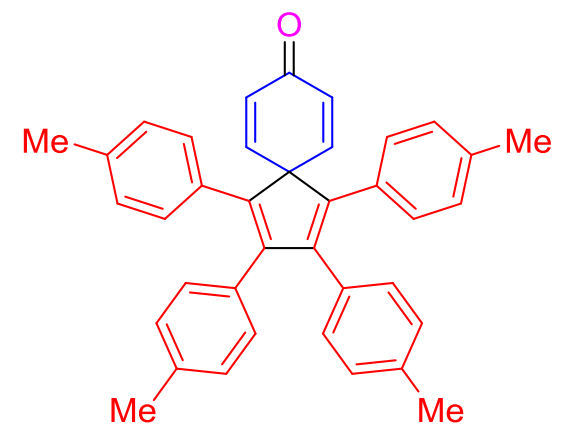

1,2,3,4-Tetra-p-tolylspiro[4.5]deca-1,3,6,9-tetraen-8-one $\quad(3 \mathrm{~m}):^{[3]}$ The title compound was prepared via the general procedure from anisole 1a $(2.0 \mathrm{~mL})$ and 1,2-di-p-tolylethyne $\mathbf{2 b}(0.4 \mathrm{mmol}, 82.5 \mathrm{mg})$. After purification by silica gel column chromatography (hexanes/EtOAc $=20: 1)$, the product was isolated in $78 \%$ yield $(78.7$ mg) as a yellow solid. IR (KBr) / cm $\mathrm{cm}^{-1}: 3022,2918,1658,1604,1594,1505,1299$, 
824. ${ }^{1} \mathbf{H}$ NMR (500 MHz, CDCl 3$): \delta 6.99-6.91(\mathrm{~m}, 12 \mathrm{H}), 6.85-6.77(\mathrm{~m}, 6 \mathrm{H}), 6.44(\mathrm{~d}$, $J=9.9 \mathrm{~Hz}, 2 \mathrm{H}), 2.29$ (s, 6H), $2.27(\mathrm{~s}, 6 \mathrm{H}) .{ }^{13} \mathbf{C}$ NMR (125 MHz, CDCl $\left.\mathbf{3}\right): \delta$ 186.4, 148.7, 147.4, 140.2, 136.9, 136.70 132.0, 131.9, 131.6, 129.8, 129.1, 128.64, 128.57, 77.3, 77.0, 76.8, 66.2, 21.3, 21.2. HRMS (ESI): calcd for $\mathrm{C}_{38} \mathrm{H}_{32} \mathrm{ONa}[\mathrm{M}+\mathrm{Na}]^{+}$ 527.2345, found 527.2354.

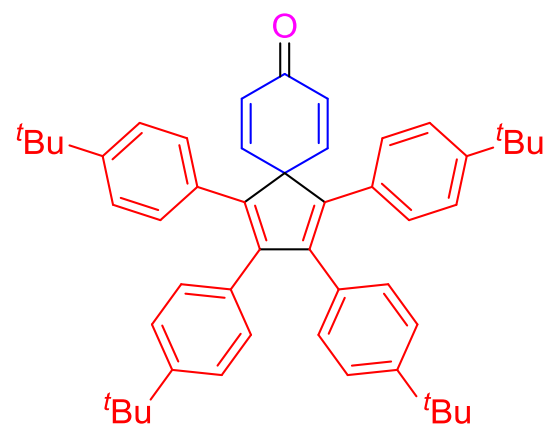

1,2,3,4-Tetrakis(4-tert-butylphenyl)spiro[4.5]deca-1,3,6,9-tetraen-8-one (3n): The title compound was prepared via the general procedure from anisole $1 \mathbf{a}(2.0 \mathrm{~mL})$ and 1,2-bis(4-(tert-butyl)phenyl)ethyne $2 \mathrm{c}(0.4 \mathrm{mmol}, 116.2 \mathrm{mg})$. After purification by silica gel column chromatography (hexanes/EtOAc $=20: 1$ ), the product was isolated in $75 \%$ yield $\left(101.0 \mathrm{mg}\right.$ ) as a yellow solid. ${ }^{\mathbf{1}} \mathbf{H}$ NMR (500 $\left.\mathbf{~ M H z}, \mathbf{C D C l}_{3}\right): \delta 7.14-7.08$ $(\mathrm{m}, 8 \mathrm{H}), 7.03(\mathrm{~d}, J=8.5 \mathrm{~Hz}, 4 \mathrm{H}), 6.88-6.78(\mathrm{~m}, 6 \mathrm{H}), 6.47(\mathrm{~d}, J=9.9 \mathrm{~Hz}, 2 \mathrm{H})$, 1.29-1.23 (m, 36H). ${ }^{\mathbf{1 3}} \mathbf{C}$ NMR (125 MHz, $\left.\mathbf{C D C l}_{3}\right): \delta 186.7,149.90,149.85,149.3$, $147.8,139.5,132.2$, 131.7, 131.5, 129.6, 128.8, 124.6, 124.5, 77.3, 77.0, 76.8, 66.2, 34.4, 31.3, 31.2. HRMS (ESI): calcd for $\mathrm{C}_{50} \mathrm{H}_{56} \mathrm{ONa}[\mathrm{M}+\mathrm{Na}]^{+} 695.4223$, found 695.4246 .

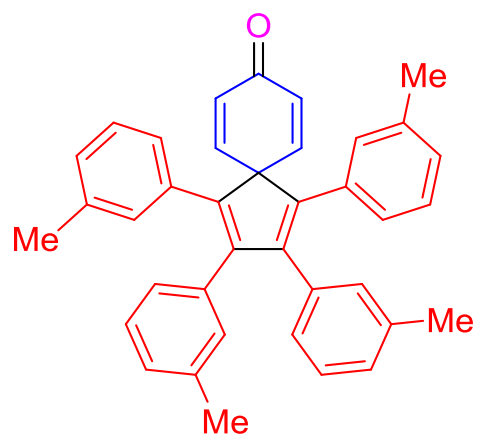

1,2,3,4-Tetra-m-tolylspiro[4.5]deca-1,3,6,9-tetraen-8-one (3o): The title compound was prepared via the general procedure from anisole $1 \mathbf{a}(2.0 \mathrm{~mL})$ and 1,2-di- $m$-tolylethyne $\mathbf{2 d}(0.4 \mathrm{mmol}, 82.5 \mathrm{mg})$. After purification by silica gel column 
chromatography (hexanes/EtOAc $=20: 1)$, the product was isolated in 68\% yield $(68.6$ $\mathrm{mg}$ ) as a yellow solid. ${ }^{1} \mathbf{H}$ NMR (500 $\left.\mathbf{~ M H z , ~} \mathbf{C D C l}_{3}\right)$ : $\delta$ 7.05-6.99 (m, 4H), 6.98-6.94 (m , 4H), $6.91(\mathrm{~s}, 2 \mathrm{H}), 6.87(\mathrm{~d}, J=7.6 \mathrm{~Hz}, 2 \mathrm{H}), 6.82(\mathrm{~d}, J=9.8 \mathrm{~Hz}, 2 \mathrm{H}), 6.75-6.69$ (m, 4H), $6.46(\mathrm{~d}, J=9.9 \mathrm{~Hz}, 2 \mathrm{H}), 2.17$ (s, 6H), 2.13 (s, 6H). ${ }^{13}$ C NMR (125 MHz, $\left.\mathbf{C D C l}_{3}\right): \delta 186.3,148.4,147.8,140.6,137.2,137.1,134.7,134.6,131.6,130.7,129.9$, $128.1,127.8,127.7,127.6,127.0,126.4,77.3,77.0,76.8,66.2,21.4,21.3$. HRMS (ESI): calcd for $\mathrm{C}_{38} \mathrm{H}_{32} \mathrm{ONa}[\mathrm{M}+\mathrm{Na}]^{+}$527.2345, found 527.2353.

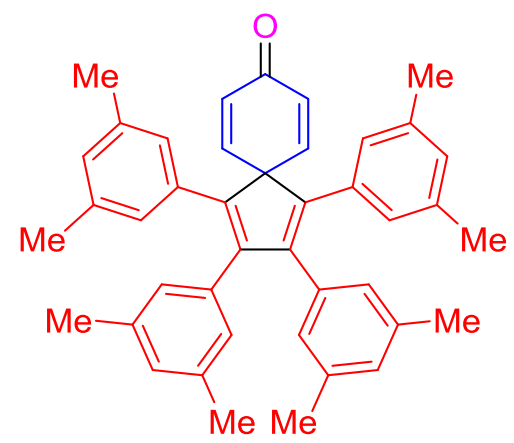

1,2,3,4-Tetrakis(3,5-dimethylphenyl)spiro[4.5]deca-1,3,6,9-tetraen-8-one

(3p):

The title compound was prepared via the general procedure from anisole $1 \mathbf{a}(2.0 \mathrm{~mL})$ and 1,2-bis(3,5-dimethylphenyl)ethyne $2 \mathbf{e}(0.4 \mathrm{mmol}, 93.7 \mathrm{mg})$. After purification by silica gel column chromatography (hexanes/EtOAc $=20: 1$ ), the product was isolated in 73\% yield (81.9 mg) as a yellow solid. ${ }^{1} \mathbf{H}$ NMR (500 $\left.\mathbf{~ M H z , ~} \mathbf{C D C l}_{3}\right): \delta 6.83-6.75$ (m, 6H), $6.71(\mathrm{~s}, 4 \mathrm{H}), 6.54(\mathrm{~s}, 4 \mathrm{H}), 6.45(\mathrm{~d}, J=9.9 \mathrm{~Hz}, 2 \mathrm{H}), 2.13(\mathrm{~s}, 12 \mathrm{H}), 2.10$ (s, 12H). ${ }^{13} \mathbf{C}$ NMR (125 MHz, $\left.\mathbf{C D C l}_{3}\right): \delta$ 186.5, 149.0, 148.0, 140.0, 136.9, 136.7, 134.8, 134.6, 131.4, 128.9, 128.5, 127.8, 127.1, 77.3, 77.0, 76.8, 66.2, 21.2, 21.1. HRMS (ESI): calcd for $\mathrm{C}_{42} \mathrm{H}_{41} \mathrm{O}[\mathrm{M}+\mathrm{H}]^{+}$561.3152, found 561.3166.

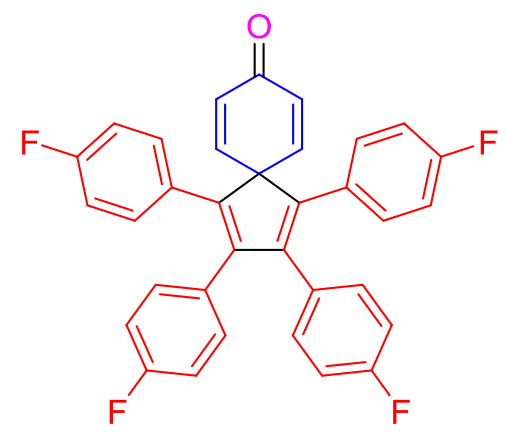

1,2,3,4-Tetrakis(4-fluorophenyl)spiro[4.5]deca-1,3,6,9-tetraen-8-one (3q): The title compound was prepared via the general procedure from anisole $1 \mathbf{a}(2.0 \mathrm{~mL})$ and 
1,2-bis(4-fluorophenyl)ethyne $\mathbf{2 f}(0.4 \mathrm{mmol}, 85.7 \mathrm{mg})$. After purification by silica gel column chromatography (hexanes/EtOAc $=20: 1$ ), the product was isolated in $71 \%$ yield $(73.9 \mathrm{mg})$ as a yellow solid. IR $(\mathrm{KBr}) / \mathrm{cm}^{-1}: 3045,1662,1599,1501,1224$, 1158, 836. ${ }^{1} \mathbf{H}$ NMR (500 MHz, $\left.\mathbf{C D C l}_{3}\right): \delta$ 7.05-7.02 (m, 4H), 6.88-6.84 (m, 12H), $6.77(\mathrm{~d}, J=10.0 \mathrm{~Hz}, 2 \mathrm{H}), 6.49(\mathrm{~d}, J=10.0 \mathrm{~Hz}, 2 \mathrm{H}) .{ }^{\mathbf{1 3}} \mathbf{C}$ NMR (125 MHz, $\left.\mathbf{C D C l}_{3}\right): \delta$ $186.1,163.5,161.6,147.5,146.8,141.1,132.7,132.1$, 132.0, 131.5, 131.4, 130.6, 115.9, 115.7, 77.3, 77.0, 76.8, 66.5. HRMS (ESI): calcd for $\mathrm{C}_{34} \mathrm{H}_{20} \mathrm{OF}_{4} \mathrm{Na}[\mathrm{M}+\mathrm{Na}]^{+}$ 543.1342 , found 543.1363 .

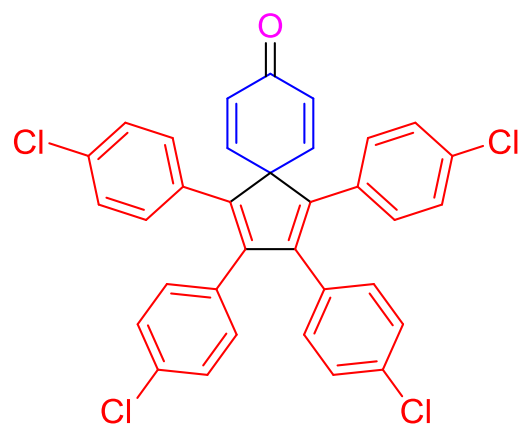

1,2,3,4-Tetrakis(4-chlorophenyl)spiro[4.5]deca-1,3,6,9-tetraen-8-one $(3 r):^{[3]}$ The title compound was prepared via the general procedure from anisole $1 \mathbf{a}(2.0 \mathrm{~mL})$ and 1,2-bis(4-chlorophenyl)ethyne $2 \mathrm{~g}(0.4 \mathrm{mmol}, 98.8 \mathrm{mg})$. After purification by silica gel column chromatography (hexanes/EtOAc $=20: 1$ ), the product was isolated in $69 \%$ yield (80.9 mg) as a yellow solid. ${ }^{1} \mathbf{H}$ NMR (500 $\left.\mathbf{M H z}, \mathbf{C D C l}_{3}\right): \delta 7.16-7.13(\mathrm{~m}, 8 \mathrm{H})$, $6.99(\mathrm{~d}, J=8.5 \mathrm{~Hz}, 4 \mathrm{H}), 6.83(\mathrm{~d}, J=8.5 \mathrm{~Hz}, 4 \mathrm{H}), 6.77$ (d, $J=10.0 \mathrm{~Hz}, 2 \mathrm{H}), 6.49$ (d, $J$ $=10.0 \mathrm{~Hz}, 2 \mathrm{H}) .{ }^{13} \mathbf{C}$ NMR (125 MHz, $\left.\mathbf{C D C l}_{3}\right): \delta$ 185.9, 146.9, 146.7, 141.7, 134.44, 134.37, 132.9, 132.8, 132.7, 131.6, 130.9, 129.11, 129.07, 77.3, 77.0, 76.8, 66.4. HRMS (ESI): calcd for $\mathrm{C}_{34} \mathrm{H}_{20} \mathrm{OCl}_{4} \mathrm{Na}[\mathrm{M}+\mathrm{Na}]^{+}$607.0160, found 607.0179.

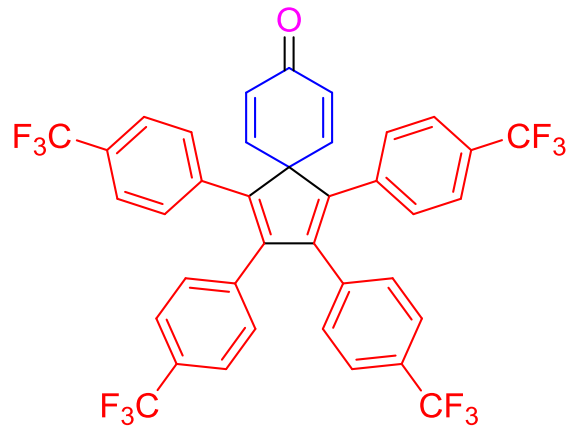

1,2,3,4-Tetrakis (4-(trifluoromethyl) phenyl) spiro [4.5] 
deca-1,3,6,9-tetraen-8-one (3s): The title compound was prepared via the general procedure from anisole $\mathbf{1 a}(2.0 \mathrm{~mL})$ and 1,2-bis(4-(trifluoromethyl)phenyl)ethyne $\mathbf{2 h}$ (0.4 mmol, $125.7 \mathrm{mg}$ ). After purification by silica gel column chromatography (hexanes/EtOAc $=20: 1)$, the product was isolated in $55 \%$ yield $(79.3 \mathrm{mg}$ ) as a yellow solid. ${ }^{1} \mathbf{H}$ NMR (500 MHz, $\left.\mathbf{C D C l}_{3}\right): \delta 7.46(\mathrm{~d}, J=8.2 \mathrm{~Hz}, 8 \mathrm{H}), 7.18(\mathrm{~d}, J=8.1 \mathrm{~Hz}$, 4H), $7.02(\mathrm{~d}, J=8.0 \mathrm{~Hz}, 4 \mathrm{H}), 6.80(\mathrm{~d}, J=9.9 \mathrm{~Hz}, 2 \mathrm{H}), 6.53(\mathrm{~d}, J=9.8 \mathrm{~Hz}, 2 \mathrm{H}) .{ }^{13} \mathbf{C}$ NMR (125 MHz, $\left.\mathbf{C D C l}_{3}\right): \delta$ 184.9, 146.6, 145.1, 142.9, 137.00, 136.96, 132.9, 130.3 $(\mathrm{q}, J=32.8 \mathrm{~Hz}), 130.2$ (q, $J=32.4 \mathrm{~Hz}), 130.0,129.4,125.49,125.47,125.44,125.40$, 125.37, $123.7(\mathrm{q}, J=270.6 \mathrm{~Hz}), 77.3,77.0,76.8,66.1$. HRMS (ESI): calcd for $\mathrm{C}_{38} \mathrm{H}_{20} \mathrm{OF}_{12} \mathrm{Na}[\mathrm{M}+\mathrm{Na}]^{+}$743.1215, found 743.1226.

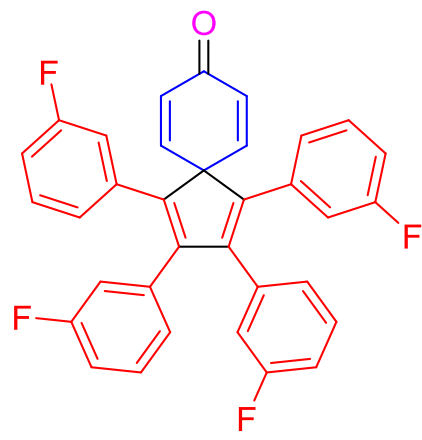

1,2,3,4-Tetrakis(3-fluorophenyl)spiro[4.5]deca-1,3,6,9-tetraen-8-one (3t): The title compound was prepared via the general procedure from anisole 1a $(2.0 \mathrm{~mL})$ and 1,2-bis(3-fluorophenyl)ethyne $2 \mathbf{i}(0.4 \mathrm{mmol}, 85.7 \mathrm{mg})$. After purification by silica gel column chromatography (hexanes/EtOAc $=20: 1$ ), the product was isolated in $65 \%$ yield (67.7 mg) as a yellow solid. ${ }^{\mathbf{1}} \mathbf{H}$ NMR (500 $\left.\mathbf{M H z}, \mathbf{C D C l}_{\mathbf{3}}\right): \delta 7.19-7.10(\mathrm{~m}, 4 \mathrm{H})$, 6.96-6.89 (m, 4H), 6.88-6.84 (m, 2H), 6.79-6.74 (m, 4H), 6.71 (d, J = 7.8 Hz, 2H), 6.66-6.58 (m, 2H), 6.54-6.48 (m, 2H). ${ }^{13} \mathbf{C}$ NMR (125 MHz, CDCl $): \delta 185.4,163.4$, 163.3, 161.4, 161.3, 146.4, 146.0, 141.6, 135.9, 135.81, 135.78, 135.7, 132.5, 129.9, $129.84,129.78,125.48,125.46,124.93,124.90,116.7,116.5,116.2,116.0,115.2$, 115.1, 115.0, 114.9, 77.3, 77.0, 76.8, 65.9. HRMS (ESI): calcd for $\mathrm{C}_{34} \mathrm{H}_{20} \mathrm{OF}_{4} \mathrm{Na}$ $[\mathrm{M}+\mathrm{Na}]^{+}$543.1342, found 543.1349. 


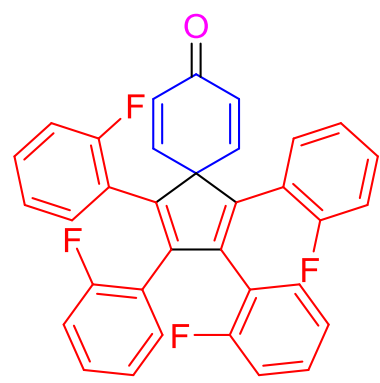

1,2,3,4-Tetrakis(2-fluorophenyl)spiro[4.5]deca-1,3,6,9-tetraen-8-one (3u): The title compound was prepared via the general procedure from anisole 1a $(2.0 \mathrm{~mL})$ and 1,2-bis(2-fluorophenyl)ethyne $\mathbf{2 j}$ ( $0.4 \mathrm{mmol}, 85.7 \mathrm{mg})$. After purification by silica gel column chromatography (hexanes/EtOAc $=20: 1$ ), the product was isolated in $60 \%$ yield (62.5 mg) as a yellow solid. ${ }^{1} \mathbf{H}$ NMR (500 $\left.\mathbf{M H z}, \mathbf{C D C l}_{3}\right): \delta 7.15-7.12(\mathrm{~m}, 5 \mathrm{H})$, 6.94-6.85 (m, 6H), 6.71-6.669 (m, 5H), 6.64-6.60 (m, 2H), 6.49 (d, J = 2.5 Hz, 2H). ${ }^{13}$ C NMR (125 MHz, CDCl 3 ): $\delta$ 185.9, 164.5, 164.4, 161.2, 161.1, 146.9, 146.5, $142.0,136.33,136.25,136.1,133.0,130.39,130.35,130.3,130.2,126.0,125.9$, $125.38,125.35,117.2,116.9,116.7,116.4,115.7,115.6,115.4,115.3,77.3,77.0,76.8$, 66.4. HRMS (ESI): calcd for $\mathrm{C}_{34} \mathrm{H}_{20} \mathrm{OF}_{4} \mathrm{Na}[\mathrm{M}+\mathrm{Na}]^{+}$543.1342, found 543.1351.

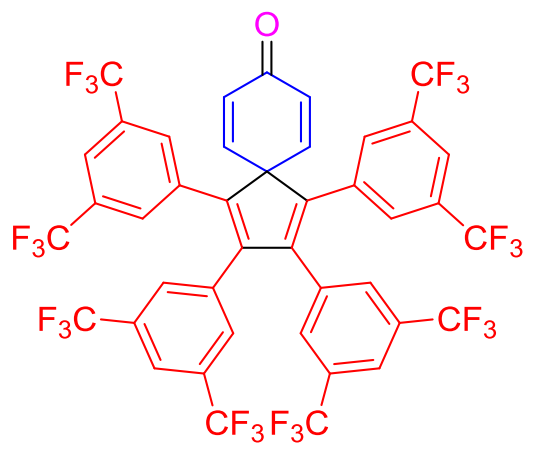

\section{1,2,3,4-Tetrakis(3,5-bis(trifluoromethyl)phenyl)spiro[4.5]deca-1,3,6,9-tetraen-8-o}

ne (3v): The title compound was prepared via the general procedure from anisole 1a (2.0 mL) and 1,2-bis(3,5-bis(trifluoromethyl)phenyl)ethyne $2 \mathbf{k}(0.4 \mathrm{mmol}, 180.1 \mathrm{mg})$. After purification by silica gel column chromatography (hexanes/EtOAc $=20: 1$ ), the product was isolated in $51 \%$ yield $(101.2 \mathrm{mg})$ as a yellow solid. ${ }^{\mathbf{1}} \mathbf{H}$ NMR (500 MHz, $\left.\mathbf{C D C l}_{3}\right): \delta 7.81(\mathrm{~d}, J=8.0 \mathrm{~Hz}, 4 \mathrm{H}), 7.52(\mathrm{~s}, 4 \mathrm{H}), 7.31(\mathrm{~s}, 4 \mathrm{H}), 6.81(\mathrm{~d}, J=10.0 \mathrm{~Hz}$, 2H), $6.66(\mathrm{~d}, J=10.0 \mathrm{~Hz}, 2 \mathrm{H}) .{ }^{13} \mathbf{C}$ NMR (125 MHz, $\left.\mathbf{C D C l}_{3}\right): \delta 183.8,145.8,144.0$, $143.0,134.7,134.6,134.5,133.4,133.2,133.1,132.8,132.7,130.1,129.4,124.4$, 
124.2, 123.3, 123.1, 121.7, 121.5, 77.3, 77.0, 76.8, 66.2. HRMS (ESI): calcd for $\mathrm{C}_{42} \mathrm{H}_{16} \mathrm{OF}_{24} \mathrm{Na}[\mathrm{M}+\mathrm{Na}]^{+}$1015.0710, found 1015.0742.

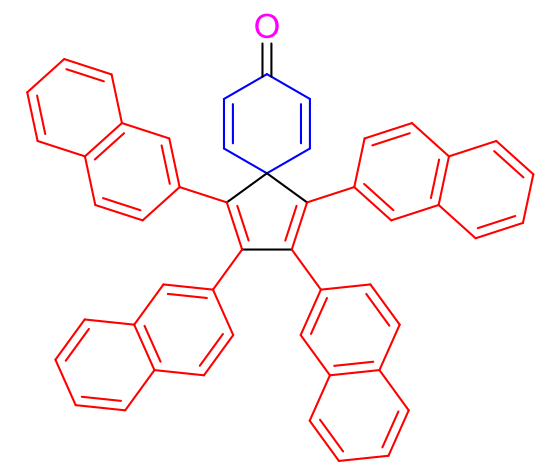

1,2,3,4-Tetra(naphthalen-2-yl)spiro[4.5]deca-1,3,6,9-tetraen-8-one $(3 w):^{[3]}$ The title compound was prepared via the general procedure from anisole 1a $(2.0 \mathrm{~mL})$ and 1,2-di(naphthalen-2-yl)ethyne $2 \mathbf{2}(0.4 \mathrm{mmol}, 111.3 \mathrm{mg})$. After purification by silica gel column chromatography (hexanes/EtOAc $=20: 1$ ), the product was isolated in 55\% yield (71.4 mg) as a yellow solid. ${ }^{\mathbf{1}} \mathbf{H}$ NMR (500 $\left.\mathbf{~ M H z , ~} \mathbf{C D C l}_{3}\right): \delta 7.79(\mathrm{~s}, 2 \mathrm{H}), 7.71$ (t, $J=7.9 \mathrm{~Hz}, 4 \mathrm{H}), 7.63(\mathrm{~d}, J=7.5 \mathrm{~Hz}, 2 \mathrm{H}), 7.58-7.51(\mathrm{~m}, 6 \mathrm{H}), 7.50-7.38(\mathrm{~m}, 10 \mathrm{H})$, 7.36-7.31 (m, 2H), $7.13(\mathrm{t}, J=9.2 \mathrm{~Hz}, 2 \mathrm{H}), 7.06(\mathrm{~d}, J=9.6 \mathrm{~Hz}, 2 \mathrm{H}), 6.55(\mathrm{~d}, J=9.7$ Hz, 2H). ${ }^{13} \mathbf{C}$ NMR (125 MHz, $\left.\mathbf{C D C l}_{3}\right): \delta$ 186.5, 148.6, 148.4, 142.1, 133.4, 132.90, $132.88,132.7,132.6,132.5,129.9,128.63$, 128.58, 128.2, 128.04, 128.01, 127.98, 127.94, 126.7, 126.6, 126.5, 126.4, 77.7, 77.5, 77.2, 67.1. HRMS (ESI): calcd for $\mathrm{C}_{50} \mathrm{H}_{32} \mathrm{ONa}[\mathrm{M}+\mathrm{Na}]^{+}$671.2345, found 671.2369.
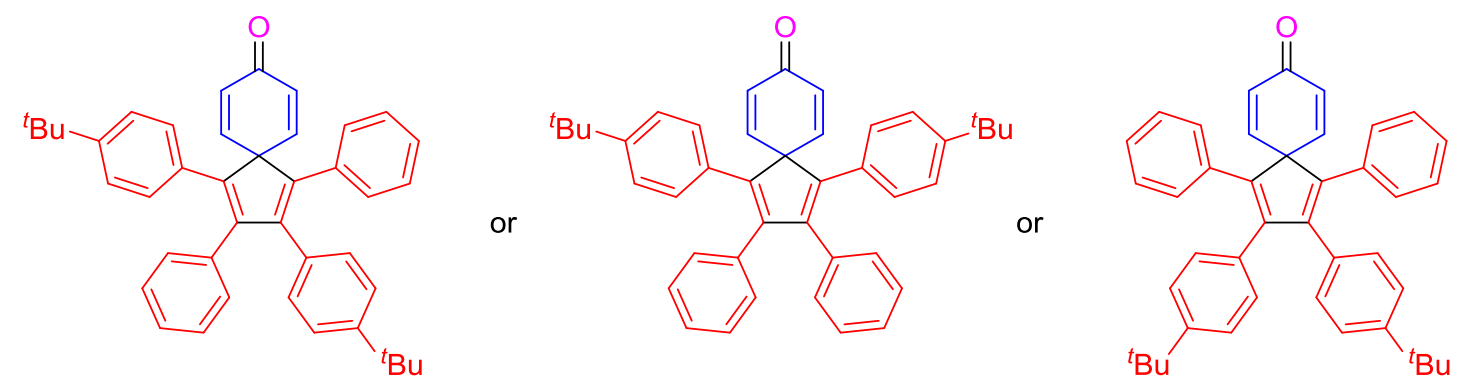

1,3-Bis(4-tert-butylphenyl)-2,4-diphenylspiro[4.5]deca-1,3,6,9-tetraen-8-one or 1,4-Bis(4-tert-butylphenyl)-2,3-diphenylspiro[4.5]deca-1,3,6,9-tetraen-8-one or 2,3-Bis(4-tert-butylphenyl)-1,4-diphenylspiro[4.5]deca-1,3,6,9-tetraen-8-one (3x):

The title compound was prepared via the general procedure from anisole $\mathbf{1 a}(2.0 \mathrm{~mL})$ and 1-(tert-butyl)-4-(phenylethynyl)benzene $\mathbf{2 m} \quad(0.4 \mathrm{mmol}, 93.7 \mathrm{mg}) . \quad$ After purification by silica gel column chromatography (hexanes/EtOAc $=20: 1$ ), the 
product was isolated in $61 \%$ yield $(68.3 \mathrm{mg})$ as a yellow oil, regioisomer ratio $\approx$ $1: 1: 1$

${ }^{1} \mathbf{H}$ NMR (500 MHz, $\left.\mathbf{C D C l}_{3}\right)$ : 7.17-7.11 (m, 10H), 7.01-6.99 (m, 4H), 6.94-6.92 (m, 4H), 6.85-6.83 (m, 2H), 6.49-6.47 (m, 2H), 1.25 (s, 18H). ${ }^{13}$ C NMR (125 MHz, $\left.\mathbf{C D C l}_{3}\right): \delta 187.1,150.6,149.2,147.9,141.0,135.6,132.2,131.9,130.4,129.28$, 129.25, 128.3, 127.5, 125.2, 66.6, 34.9, 31.6. HRMS (ESI): calcd for $\mathrm{C}_{42} \mathrm{H}_{40} \mathrm{ONa}$ $[\mathrm{M}+\mathrm{Na}]^{+}$583.2971, found 583.2970.

${ }^{1}$ H NMR (500 MHz, CDCl $)$ ) 7.16-7.09 (m, 14H), 6.86-6.81 (m, 6H), 6.46-6.44 (m, 2H). ${ }^{13} \mathbf{C}$ NMR (125 MHz, $\left.\mathbf{C D C l}_{3}\right): \delta$ 186.4, 150.1, 148.5, 147.9, 140.3, 134.8, 131.7, 131.6, 130.0, 129.6, 129.5, 129.3, 127.9, 127.2, 124.6, 66.2, 34.5, 31.3, 31.2. HRMS (ESI): calcd for $\mathrm{C}_{42} \mathrm{H}_{40} \mathrm{ONa}[\mathrm{M}+\mathrm{Na}]^{+}$583.2971, found 583.2970.

${ }^{1}$ H NMR (500 MHz, CDCl 3$)$ : 7.18-7.11 (m, 12H), 7.10-7.07 (m, 2H), 7.02-7.00 (m, 2H), 6.95-6.80 (m, 2H), 6.47-6.45 (m, 2H). ${ }^{13} \mathbf{C}$ NMR (125 MHz, CDCl $): \delta$ 187.0, $150.61,150.56,149.1,148.3,147.8,141.2$, 140.5, 135.6, 135.2, 132.1, 131.99, 131.97, $130.5,130.4,130.3,130.0,129.9,129.3,129.2$, 128.29, 128.26, 127.7, 127.5, 125.3, 125.1, 66.4, 34.9, 31.7, 31.6. HRMS (ESI): calcd for $\mathrm{C}_{42} \mathrm{H}_{40} \mathrm{ONa}[\mathrm{M}+\mathrm{Na}]^{+}$583.2971, found 583.2970.
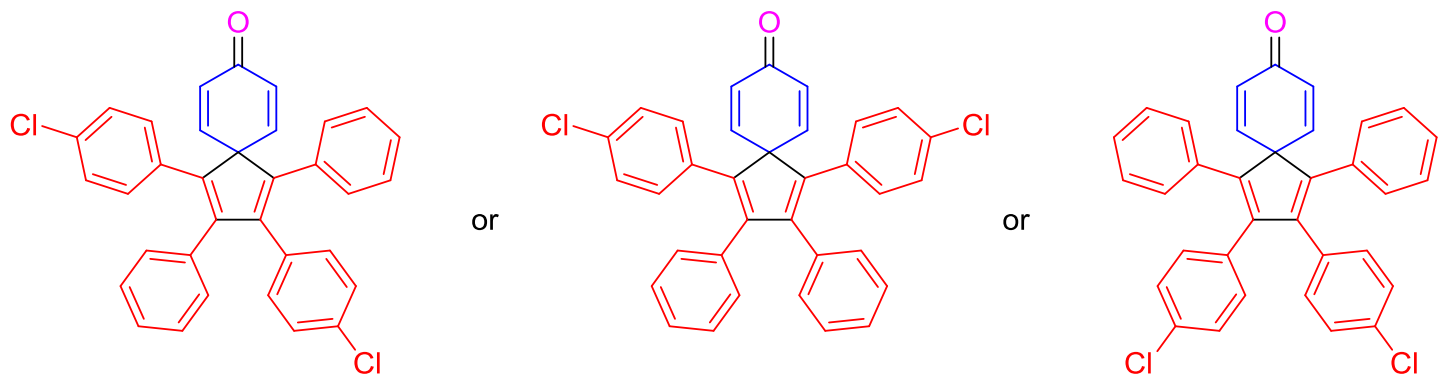

1,3-Bis(4-chlorophenyl)-2,4-diphenylspiro[4.5]deca-1,3,6,9-tetraen-8-one or 1,4-Bis(4-chlorophenyl)-2,3-diphenylspiro[4.5]deca-1,3,6,9-tetraen-8-one or 2,3-Bis(4-chlorophenyl)-1,4-diphenylspiro[4.5]deca-1,3,6,9-tetraen-8-one (3y):

The title compound was prepared via the general procedure from anisole 1a $(2.0 \mathrm{~mL})$ and 1-chloro-4-(phenylethynyl)benzene $2 \mathrm{n}(0.4 \mathrm{mmol}, 85.1 \mathrm{mg})$. After purification by silica gel column chromatography (hexanes/EtOAc $=20: 1$ ), the product was isolated in $57 \%$ yield $(59.0 \mathrm{mg})$ as a pale-yellow solid, regioisomer ratio $\approx 1: 1: 1$. 
${ }^{1}$ H NMR (500 MHz, CDCl $)$ ): $\delta$ 7.22-7.10 (m, 10H), 7.05-7.00 (m, 4H), 6.90-6.88 (m, 4H), 6.80-6.78 (m, 2H), 6.50-6.48 (m, 2H). ${ }^{13} \mathbf{C}$ NMR (125 MHz, CDCl 3 ): $\delta$ 186.2 , 148.6, 147.9, 140.5, 134.6, 134.0, 133.3, 132.6, 131.0, 130.3, 128.8, 128.6, 128.1, 66.3. HRMS (ESI): calcd for $\mathrm{C}_{34} \mathrm{H}_{23} \mathrm{OCl}_{2}[\mathrm{M}+\mathrm{H}]^{+}$517.1052, found 517.1061. ${ }^{1}$ H NMR (500 MHz, CDCl $)$ ): $\delta$ 7.22-7.18 (m, 6H), 7.17-7.15 (m, 4H), 7.11-7.08 (m, 2H), 7.06-7.00 (m, 2H), 6.92-6.90 (m, 2H), 6.83-6.77 (m, 4H), 6.47 (d, J = 9.6 Hz, 2H). ${ }^{13} \mathbf{C}$ NMR (125 MHz, $\left.\mathbf{C D C l}_{3}\right): \delta$ 186.3, 147.7, 146.6, 134.5, 133.9, 133.8, 133.3, 133.2 , 132.5, 131.7, 130.9, 130.8, 130.4, 130.23, 130.15, 129.71, 129.67, 129.6, $128.81,128.78,128.70,128.66,128.62,128.57,128.4,128.3,128.2$, 66.5. HRMS (ESI): calcd for $\mathrm{C}_{34} \mathrm{H}_{23} \mathrm{OCl}_{2}[\mathrm{M}+\mathrm{H}]^{+}$517.1052, found 517.1061.
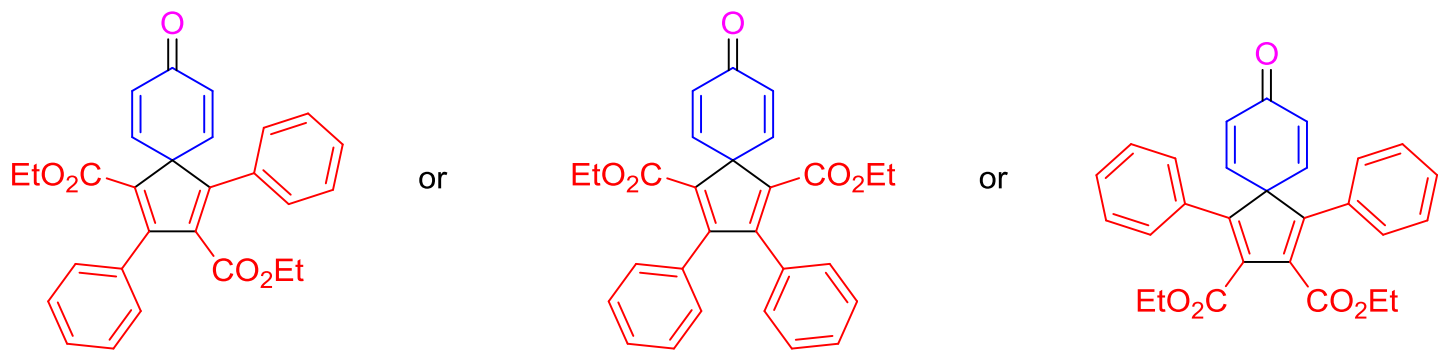

Diethyl 8-oxo-2,4-diphenylspiro[4.5]deca-1,3,6,9-tetraene-1,3-dicarboxylate or

Diethyl 8-oxo-1,4-diphenylspiro[4.5]deca-1,3,6,9-tetraene-2,3-dicarboxylate or Diethyl 8-oxo-2,3-diphenylspiro[4.5]deca-1,3,6,9-tetraene-1,4-dicarboxylate (3z):

The title compound was prepared via the general procedure from anisole 1a $(2.0 \mathrm{~mL})$ and ethyl 3-phenylpropiolate $20(0.4 \mathrm{mmol}, 69.7 \mathrm{mg})$. After purification by silica gel column chromatography (hexanes/EtOAc $=20: 1$ ), the product was isolated in $45 \%$ yield $(39.6 \mathrm{mg})$ as a yellow oil, regioisomer ratio $\approx 1: 2: 1$.

${ }^{1}$ H NMR (500 MHz, $\left.\mathbf{C D C l}_{3}\right): \delta 7.46(\mathrm{dd}, J=7.5,2.0 \mathrm{~Hz}, 1 \mathrm{H}), 7.38-7.35(\mathrm{~m}$, 2H),7.16-7.10 (m, 7H), 7.06-7.00 (m, 2H), 6.93-6.92 (m, 1H), 6.80-6.78 (m, 1H), 6.70-6.65 (m, 1H), 4.93-3.04 (m, 1H), 3.75-3.70 (m, 2H), 3.68-3.63 (m, 1H), 0.94-0.88 (m, 2H), 0.78-0.68 (m, 4H). ${ }^{13}$ C NMR (125 MHz, CDCl $): \delta 168.9,168.8$, 168.7, 168.6, 168.2, 167.9, 167.8, 158.9, 158.8, 141.23, 141.19, 140.3, 140.0, 139.6, 139.1, 138.79, 138.76, 138.6, 138.5, 138.4, 138.3, 138.20, 138.15, 138.0, 137.9, 137.8, $137.7,137.2$, 136.9, 136.3, 135.6, 135.1, 134.6, 131.7, 131.6, 131.5, 131.3, 130.7, $130.6,130.5,130.4,130.3,130.2$, 129.6, 129.3 , 128.5, 128.42, 128.36, 128.31, 127.97 , 
$127.95,127.87,127.77,127.6,127.46,127.45,127.3,126.4,113.4,113.3,62.0,61.4$ $61.26,61.25,61.23,55.52,55.51,13.95,13.92,13.91,13.84,13.83,13.79$. HRMS (ESI): calcd for $\mathrm{C}_{28} \mathrm{H}_{25} \mathrm{O}_{5}[\mathrm{M}+\mathrm{H}]^{+} 441.1622$, found 441.1630 .<smiles>CC=C1C(CC)=C(CC)C12C=CC(=O)C=C2</smiles>

1,2,3,4-Tetraethylspiro [4.5] deca-1,3,6,9-tetraen-8-one was prepared following the general procedure from anisole $\mathbf{1 a}(2.0 \mathrm{~mL})$ and hex-3-yne $\mathbf{2 p}(0.4 \mathrm{mmol}, 32.9 \mathrm{mg})$. Only trace product was formed, which was detected by GC-MS. HRMS (ESI): calcd for $\mathrm{C}_{18} \mathrm{H}_{25} \mathrm{O}[\mathrm{M}+\mathrm{H}]^{+}$257.1900, found 257.1890.

\section{Gram scale reaction.}
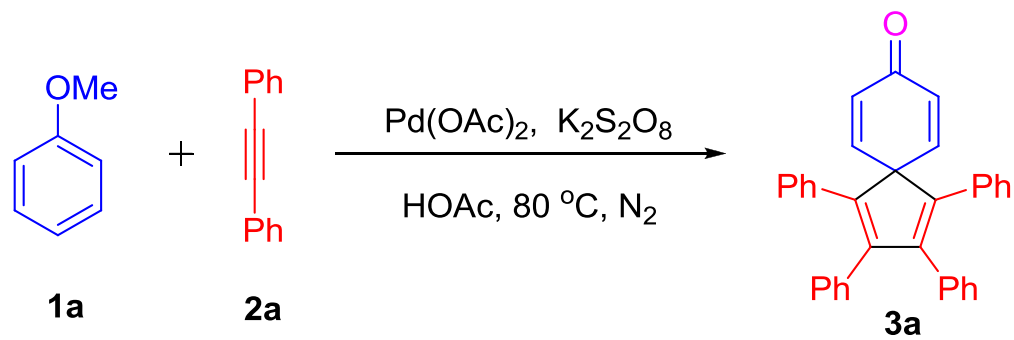

According to the general procedure: To a solution of diphenylacetylene $\mathbf{2 a}(1.78 \mathrm{~g}, 10$ mmol) in anisole 1a (50.0 mL), palladium (II) acetate (224.5 mg, $10 \mathrm{~mol} \%)$, HOAc $(572 \mathrm{uL}, 10.0 \mathrm{mmol})$ and potassium persulfate $(5.41 \mathrm{~g}, 20.0 \mathrm{mmol})$ were added. The reaction mixture was stirred at $80{ }^{\circ} \mathrm{C}$ in an oil bath under $\mathrm{N}_{2}$ until $2 \mathbf{a}$ was gone (48 h, monitored by TLC). Work-up: The reaction mixture was cooled down to room temperature. Solvent was removed under ScanVac CoolSafe Freeze Dryer. The crude product was pre-adsorbed on silica gel and purified by flash chromatography (silica gel, EtOAc/hexanes = 1:20) to afford pure product 3aa in moderate yield (1.32 g, $60 \%)$ as a yellow solid. 


\section{Intermolecular competition experiments.}

Intermolecular competition experiments with two different anisoles (1b and 1c)

$$
\begin{aligned}
& \mathbf{1 b}+\mathbf{1 c}+\mathbf{2 a} \stackrel{\text { "standard condition" }}{\stackrel{\text { inseperable mixture }}{\longrightarrow}} \\
& 3 b / 3 c=5: 3 \text { based on }{ }^{1} \mathrm{H} \text { NMR }
\end{aligned}
$$

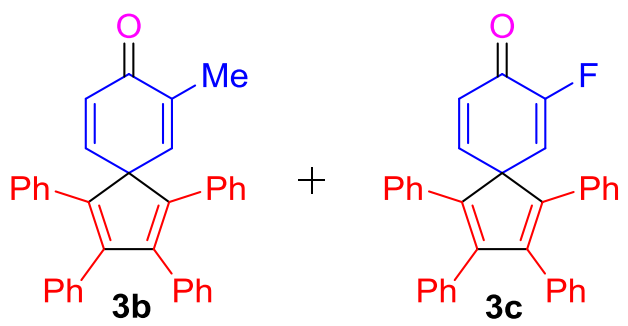

According to the general procedure: To a solution of diphenylacetylene $\mathbf{2 a}(71.2 \mathrm{mg}$, $0.4 \mathrm{mmol})$ in $\mathbf{1 b}(2.0 \mathrm{~mL})$ and $\mathbf{1 c}(2.0 \mathrm{~mL})$, palladium (II) acetate $(8.9 \mathrm{mg}, 10 \mathrm{~mol} \%)$, HOAc (24 uL, $0.4 \mathrm{mmol})$ and potassium persulfate $(216.3 \mathrm{mg}, 0.8 \mathrm{mmol})$ were added. The reaction mixture was stirred at $80{ }^{\circ} \mathrm{C}$ in an oil bath under $\mathrm{N}_{2}$ for $24 \mathrm{~h}$. Work-up: The reaction mixture was cooled down to room temperature. Solvent was removed under ScanVac CoolSafe Freeze Dryer. The crude product was pre-adsorbed on silica gel and purified by flash chromatography (silica gel, EtOAc/hexanes $=1: 20$ ) to afford an inseparable mixture of $\mathbf{3 b}$ and $\mathbf{3} \mathbf{c}$ in a rough ratio of 5:3 (132 $\mathrm{mg})$ based on its ${ }^{1} \mathrm{H}$ NMR spectrum (as shown below, the typical peaks were assigned based on the pure ${ }^{1} \mathrm{H}$ NMR spectra of $\mathbf{3 b}$ and $\mathbf{3 c}$, respectively).

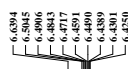

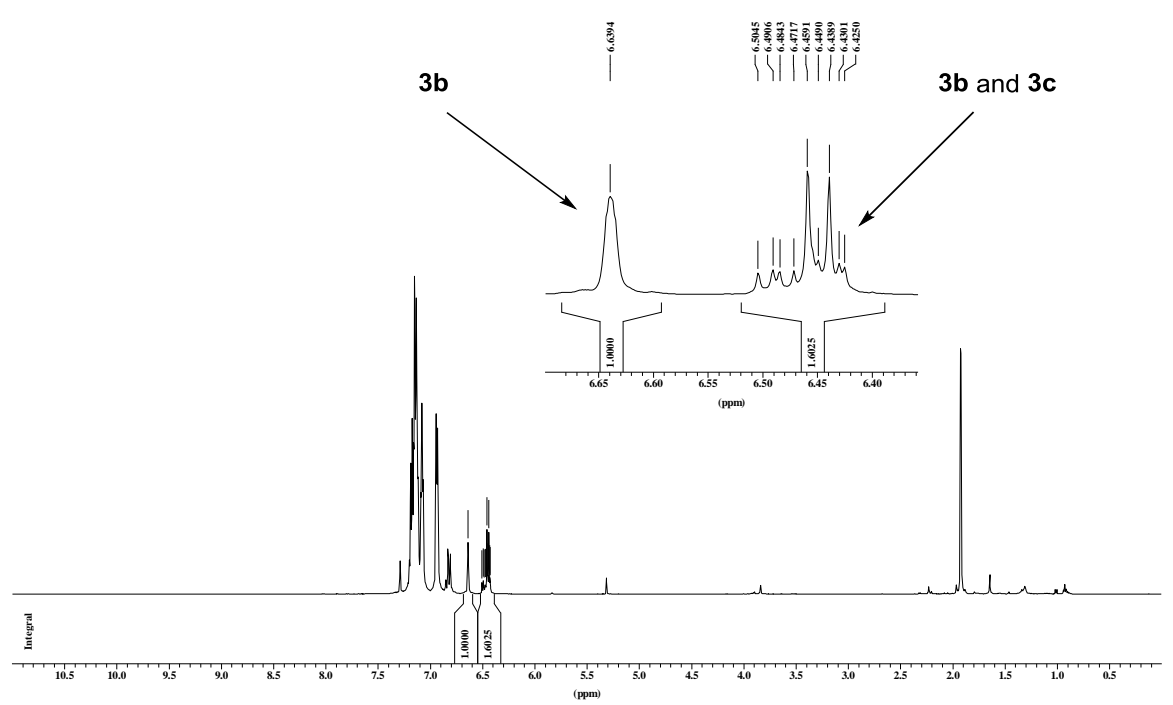


Intermolecular competition experiments with two different alkynes (2b and $2 \mathrm{e}$ )
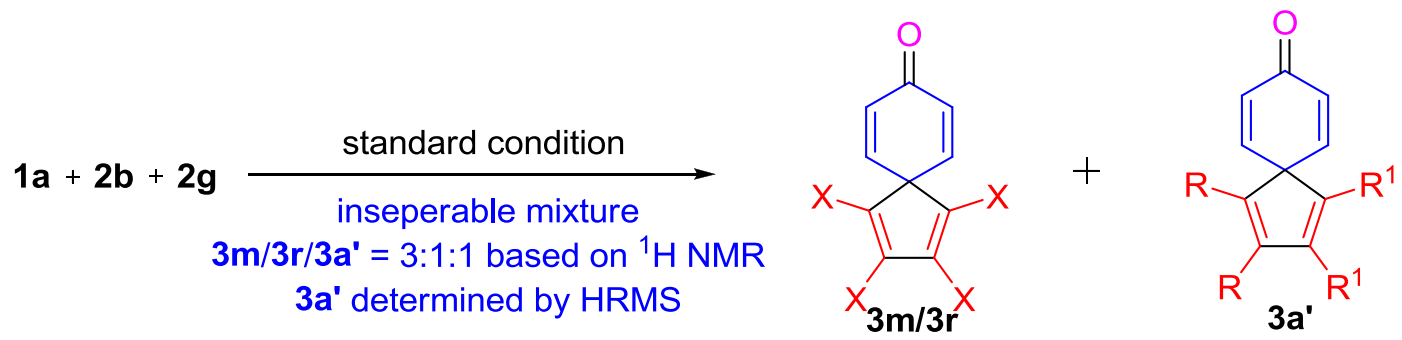

$3 \mathrm{~m}, \mathrm{X}=4-\mathrm{Me}-\mathrm{Ph} ; 3 \mathrm{r}, \mathrm{X}=4-\mathrm{Cl}-\mathrm{Ph}$ $3 a^{\prime}, \mathrm{R}=4-\mathrm{Me}-\mathrm{Ph}, \mathrm{R}^{1}=4-\mathrm{Cl}-\mathrm{Ph}$

According to the general procedure: To a solution of alkyne $\mathbf{2 b}(82.5 \mathrm{mg}, 0.4 \mathrm{mmol})$ and $2 \mathbf{c}(98.8 \mathrm{mg}, 0.4 \mathrm{mmol})$ in anisole $1 \mathbf{a}(2.0 \mathrm{~mL})$, palladium (II) acetate $(8.9 \mathrm{mg}, 10$ mol\%), HOAc (48 uL, $0.8 \mathrm{mmol})$ and potassium persulfate $(216.3 \mathrm{mg}, 0.8 \mathrm{mmol})$ were added. The reaction mixture was stirred at $80{ }^{\circ} \mathrm{C}$ in an oil bath under $\mathrm{N}_{2}$ for $12 \mathrm{~h}$. Work-up: The reaction mixture was cooled down to room temperature. Solvent was removed under ScanVac CoolSafe Freeze Dryer. The crude product was pre-adsorbed on silica gel and purified by flash chromatography (silica gel, EtOAc/hexanes $=1: 20$ ) to afford an inseparable mixture of $\mathbf{3 m}, \mathbf{3 r}$ and $\mathbf{3} \mathbf{a}^{\prime}$ in a rough ratio of 3:1:1 (119 $\left.\mathbf{m g}\right)$ based on its ${ }^{1} \mathrm{H}$ NMR spectrum. (As shown below. The typical peaks of $\mathbf{3 m}$ and $\mathbf{3 r}$ were assigned based on the pure ${ }^{1} \mathrm{H}$ NMR spectra of $\mathbf{3 m}$ and $\mathbf{3 r}$, respectively. $\mathbf{3} \mathbf{a}^{\mathbf{\prime}}$ was detected by HRMS (ESI) and its typical peaks were attributed arbitrarily).

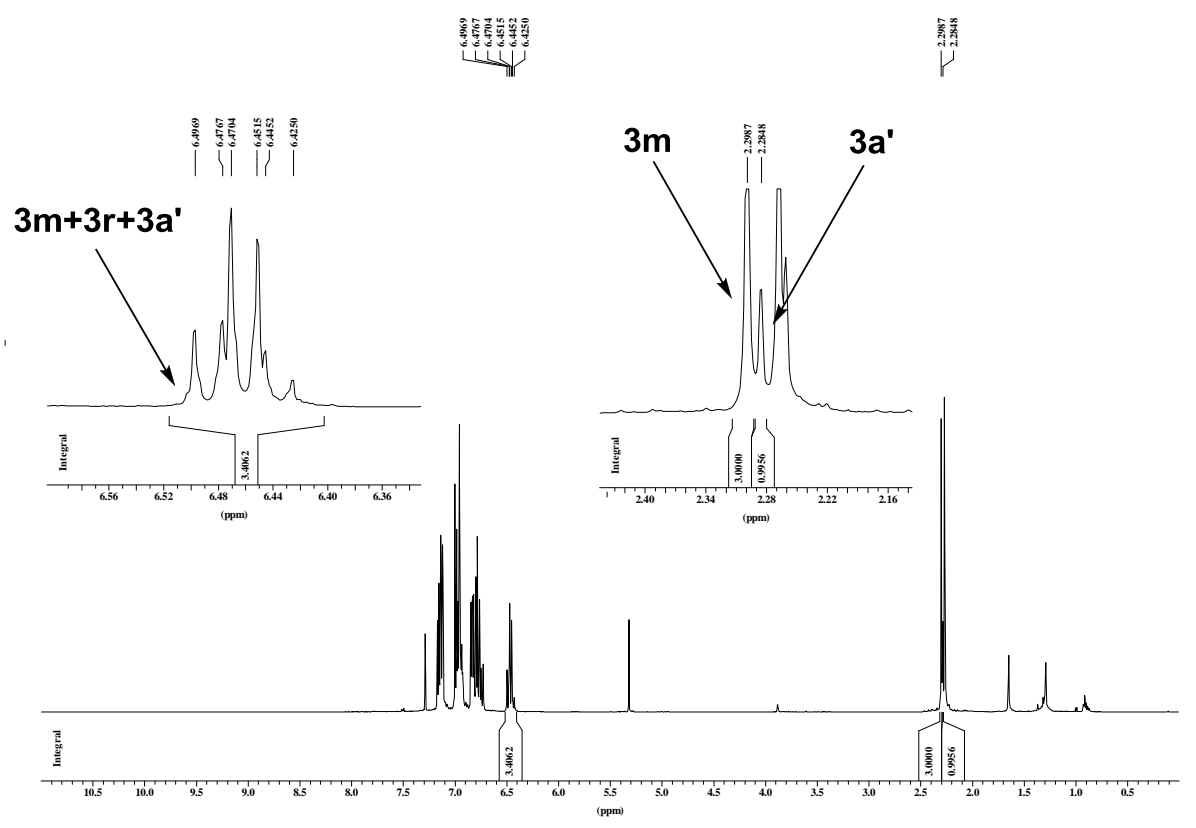




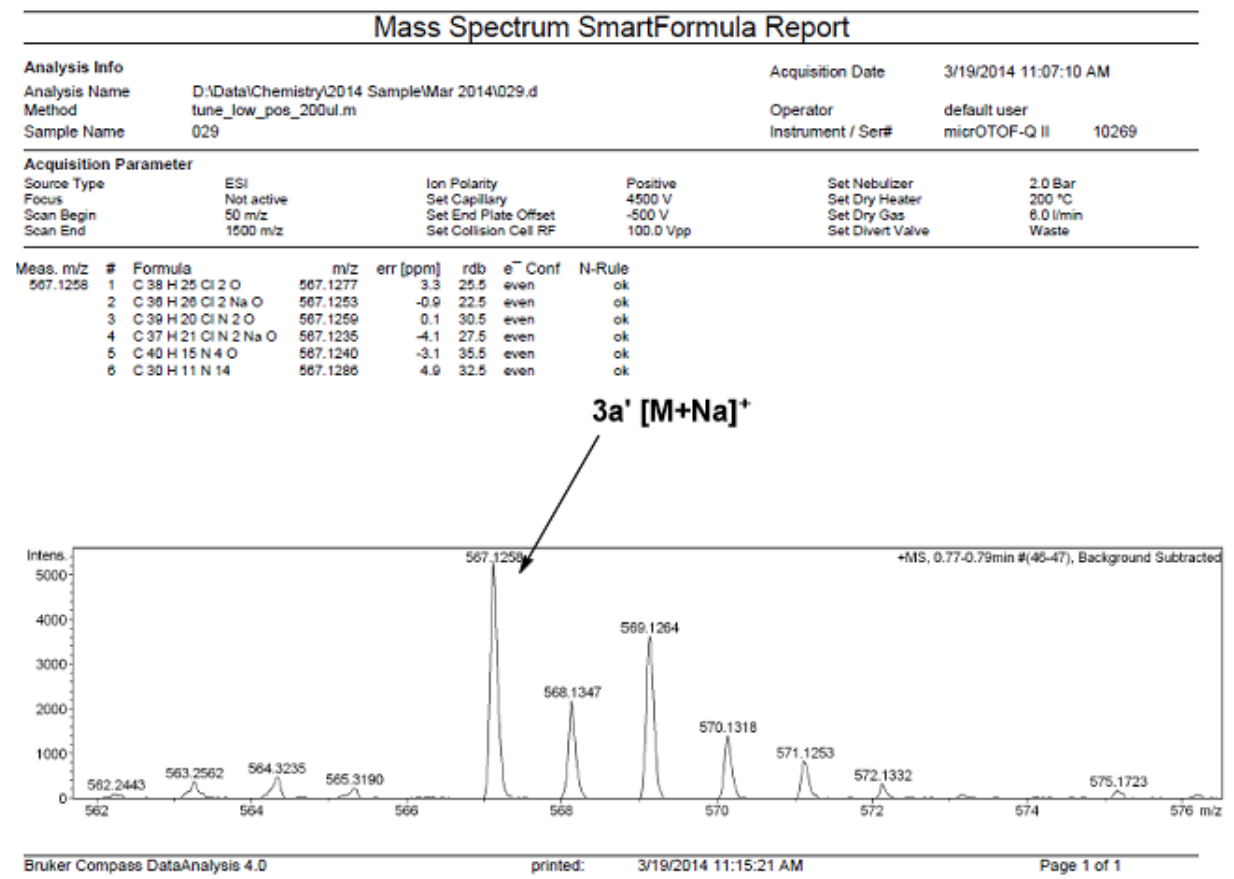

\section{KIE experiments.}

Kinetic isotope effects determined from two parallel reactions

$$
\begin{aligned}
& \mathbf{1 a}+\mathbf{2 a} \frac{\text { "standard condition" }}{3 \mathrm{~h}, 7.5 \mathrm{mg}, 33 \%} \mathbf{3 a} \\
& k_{H} / k_{D}=2.2 \\
& {\left[\mathbf{D}_{5}\right]-1 \mathbf{a}+2 \mathbf{a} \frac{\text { "standard condition" }}{3 \mathrm{~h}, 3.5 \mathrm{mg}, 15 \%}\left[\mathbf{D}_{4}\right]-3 \mathbf{a}}
\end{aligned}
$$

Two sets of reactions were carried out in a parallel manner following the general procedure for $3 \mathrm{~h}$, in each case alkyne $\mathbf{2 a}$ was allowed to react with $\mathbf{1 a}$ and $\left[\mathbf{D}_{5}\right]-\mathbf{1 a}$. The value of $\boldsymbol{k}_{\boldsymbol{H}} / \boldsymbol{k}_{\boldsymbol{D}}=2.2$ was calculated according to the isolated yields of $\mathbf{3 a}$ and $\left[\mathbf{D}_{4}\right]-3 \mathbf{a}$ from the two reactions.

Kinetic isotope effects determined from an intermolecular competition

$$
\begin{aligned}
& \mathbf{1} \mathbf{a}+\left[\mathbf{D}_{5}\right]-\mathbf{1} \mathbf{a}+\mathbf{2 a} \frac{\text { "standard condition" }}{6 \mathrm{~h}} \mathbf{3 a}+\left[\mathbf{D}_{4}\right]-\mathbf{3} \mathbf{a} \\
& k_{H} / k_{D}=4: 1 \\
& \text { ( }{ }^{1} \mathrm{H} \text { NMR) }
\end{aligned}
$$

According to the general procedure: To a solution of alkyne $2 \mathbf{a}(82.5 \mathrm{mg}, 0.4 \mathrm{mmol})$ in 1a $(1.0 \mathrm{~mL})$ and $\left[\mathbf{D}_{5}\right]-\mathbf{- 1 a}(1.0 \mathrm{~mL})$, palladium (II) acetate $(8.9 \mathrm{mg}, 10 \mathrm{~mol} \%)$, 
HOAc ( $24 \mathrm{uL}, 0.4 \mathrm{mmol})$ and potassium persulfate $(216.3 \mathrm{mg}, 0.8 \mathrm{mmol})$ were added. The reaction mixture was stirred at $80{ }^{\circ} \mathrm{C}$ in an oil bath under $\mathrm{N}_{2}$ for $6 \mathrm{~h}$. Work-up: The reaction mixture was cooled down to room temperature. Solvent was removed under ScanVac CoolSafe Freeze Dryer. The crude product was pre-adsorbed on silica gel and purified by flash chromatography (silica gel, EtOAc/hexanes $=1: 20$ ) to afford an inseparable mixture of $\mathbf{3 a}$ and $\left[\mathbf{D}_{4}\right]-\mathbf{3 a}$ in a rough ratio of $4: 1(13 \mathrm{mg})$ based on its ${ }^{1} \mathrm{H}$ NMR spectrum.(see below)

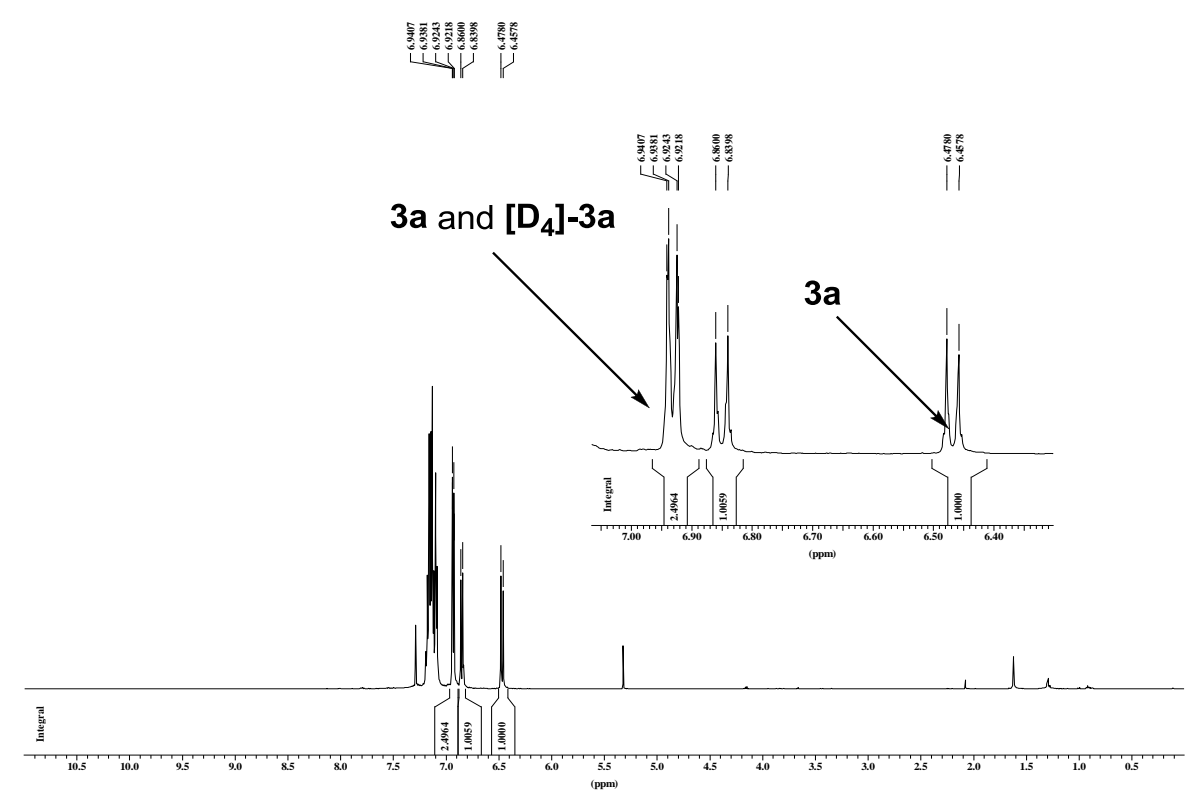

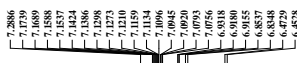

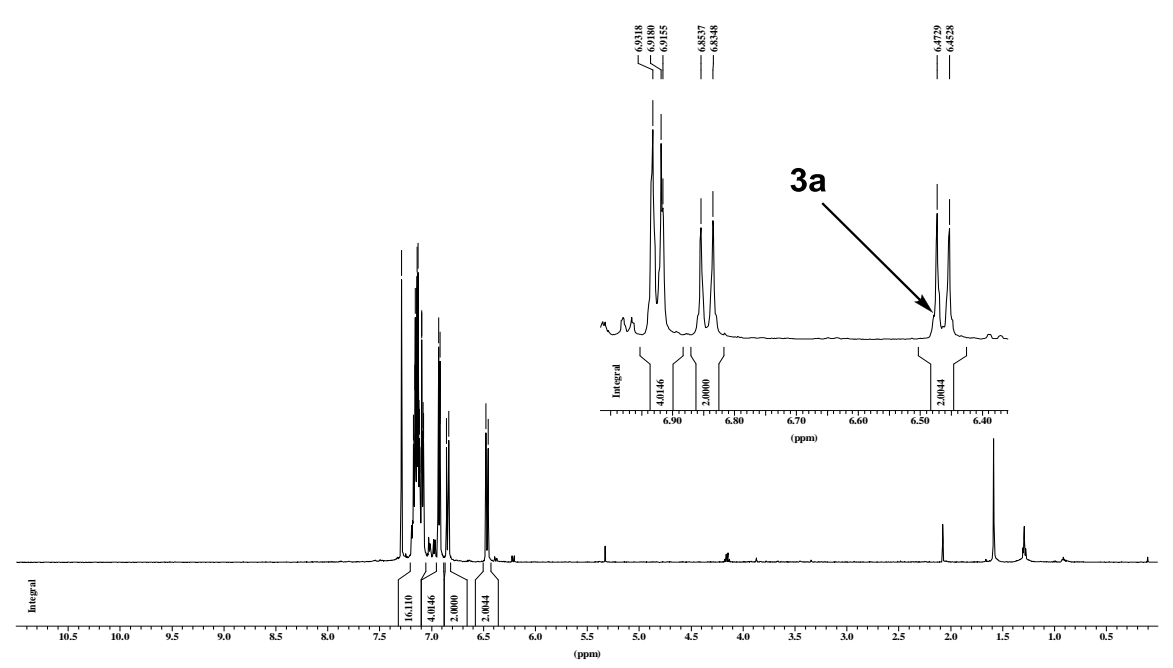




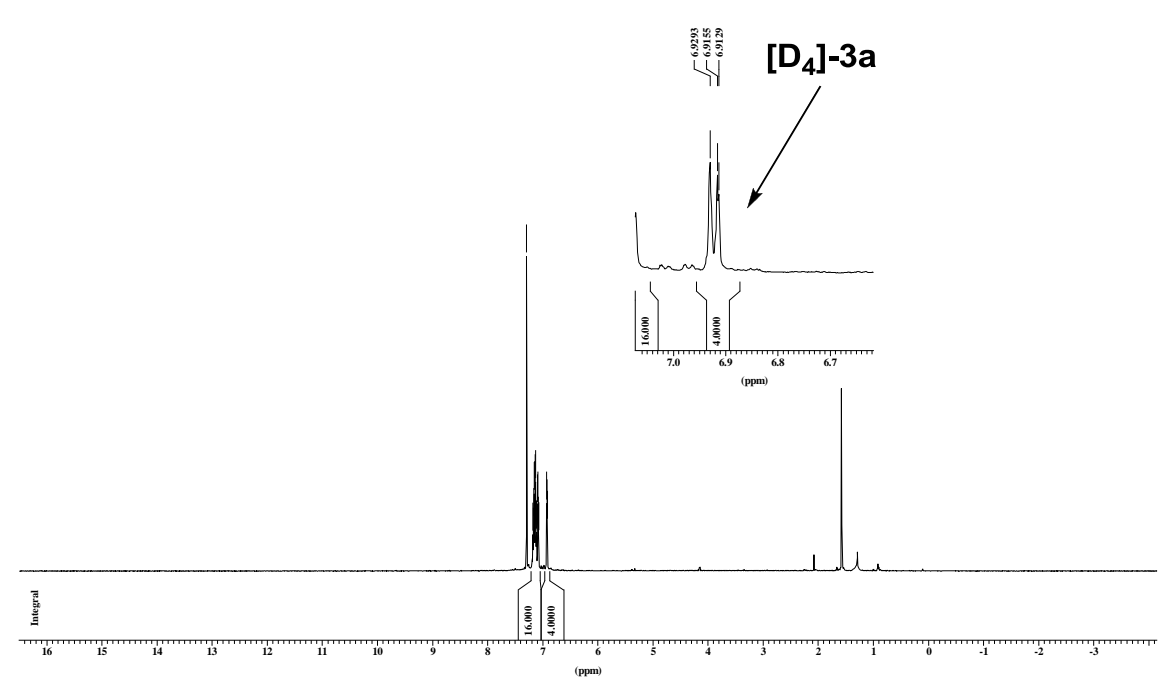

\section{Mechanistic study}

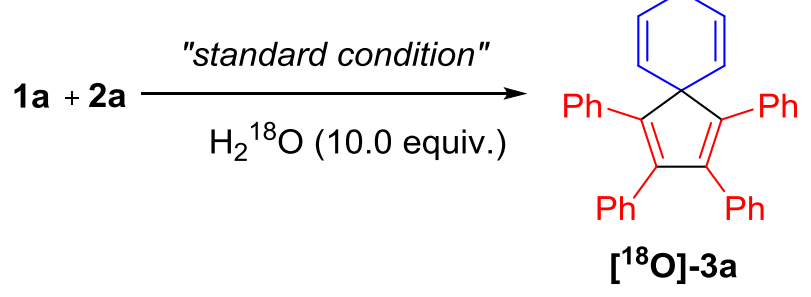

According to the general procedure: To a solution of alkyne $\mathbf{2 a}(82.5 \mathrm{mg}, 0.4 \mathrm{mmol})$ in anisole 1a $(2.0 \mathrm{~mL})$, palladium (II) acetate $(8.9 \mathrm{mg}, 10 \mathrm{~mol} \%)$, HOAc (24 uL, 0.4 mmol), potassium persulfate $(216.3 \mathrm{mg}, 0.8 \mathrm{mmol})$ and $\mathrm{H}_{2}{ }^{18} \mathrm{O}(80.0 \mathrm{mg}, 4.0 \mathrm{mmol})$ were added. The reaction mixture was stirred at $80{ }^{\circ} \mathrm{C}$ in an oil bath under $\mathrm{N}_{2}$ for $24 \mathrm{~h}$. Work-up: The reaction mixture was cooled down to room temperature. Solvent was removed under ScanVac CoolSafe Freeze Dryer. The crude product was pre-adsorbed on silica gel and purified by flash chromatography (silica gel, EtOAc/hexanes $=1: 20$ ) to obtain $\left[{ }^{18} \mathbf{O}\right]-3 \mathbf{a}$ in $65 \%$ yield, which was confirmed by HRMS (ESI). (As shown below) 


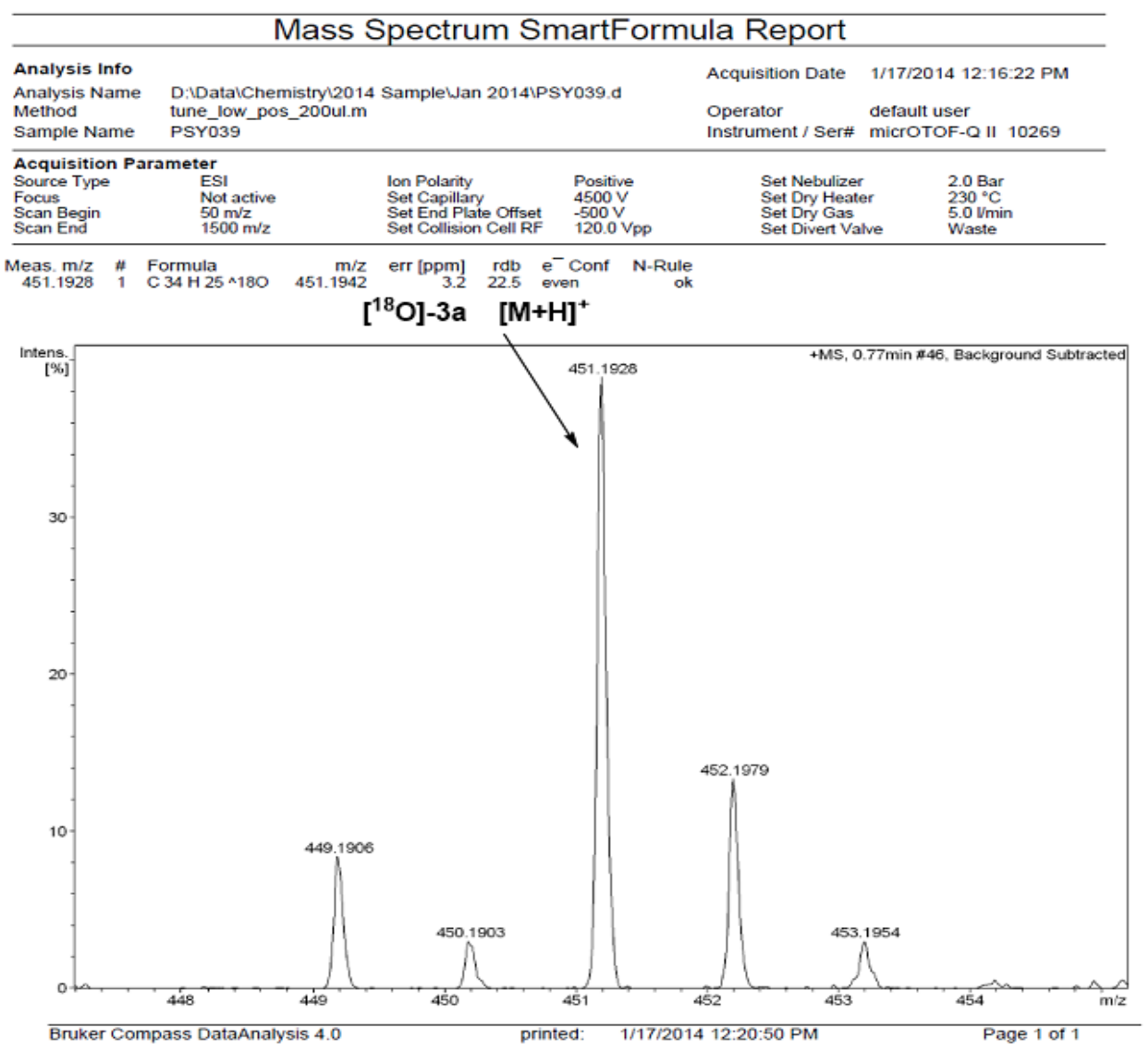

\section{Synthetic transformations of 3a.}

\section{Preparation and characterization of compound 6}

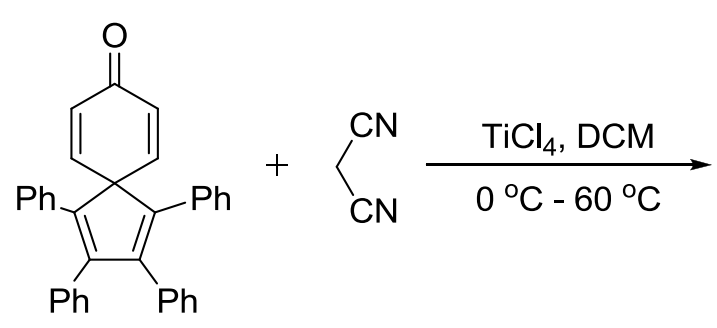<smiles>N#CC(C#N)=C1C=CC2(C=C1)C(c1ccccc1)=C(c1ccccc1)C(c1ccccc1)=C2c1ccccc1</smiles>

To a mixture of $3 \mathbf{a}(44.8 \mathrm{mg}, 0.1 \mathrm{mmol})$ and malononitrile $(0.6 \mathrm{mmol})$, in anhydrous $\operatorname{DCM}(2.0 \mathrm{~mL}), \mathrm{TiCl}_{4}(0.3 \mathrm{mmol})$ was added at $0{ }^{\circ} \mathrm{C}$ under $\mathrm{N}_{2}$ protected. After stirring $0{ }^{\circ} \mathrm{C}$ for $0.5 \mathrm{~h}$ under $\mathrm{N}_{2}$, the mixture was moved to $60{ }^{\circ} \mathrm{C}$ in an oil bath for another 12 h. Work-up: The crude reaction mixture was poured into $\mathrm{H}_{2} \mathrm{O}$ and extracted with DCM three times. The combined organic phases were dried over anhydrous $\mathrm{MgSO}_{4}$, filtered and concentrated under reduced pressure. The crude product was purified by silica gel flash chromatography and eluted by hexanes/EtOAc $=20: 1$ then 10:1 to afford product 6 in $61 \%$ yield $\left(30.2 \mathrm{mg}\right.$ ) as a red oil. ${ }^{\mathbf{1}} \mathbf{H}$ NMR (500 $\left.\mathbf{~ M H z}, \mathbf{C D C l}_{3}\right)$ : $\delta$ 
7.22-7.10 (m, 12H), $7.06(\mathrm{~d}, J=8.8 \mathrm{~Hz}, 2 \mathrm{H}), 7.00(\mathrm{~d}, J=7.6 \mathrm{~Hz}, 4 \mathrm{H}), 6.90(\mathrm{~d}, J=$ $7.6 \mathrm{~Hz}, 4 \mathrm{H}), 6.69$ (d, $J=9.5 \mathrm{~Hz}, 2 \mathrm{H}) .{ }^{13} \mathbf{C} \mathbf{N M R}\left(125 \mathbf{M H z}, \mathbf{C D C l}_{3}\right): \delta$ 157.1, 148.6, $146.5,141.2,134.8,130.4,129.6,128.6,128.4,128.2,127.9,125.3,113.3,77.8,77.5$, 77.3, 68.3. HRMS (EI): calcd for $\mathrm{C}_{37} \mathrm{H}_{24} \mathrm{~N}_{2}[\mathrm{M}]^{+} 496.1939$, found 496.1942 .

\section{Preparation and characterization of compound 7}

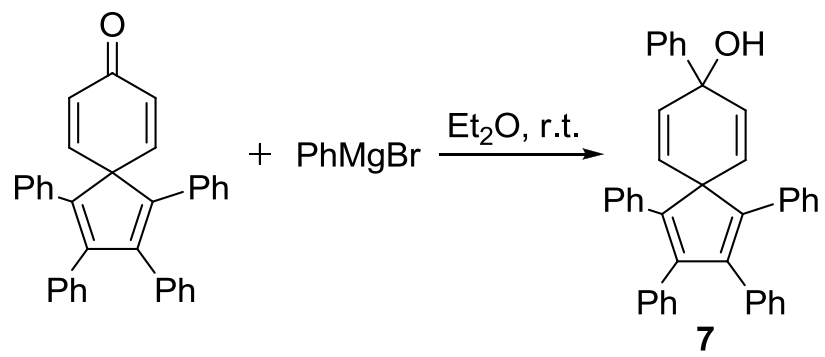

To a stirred solution of $\mathbf{3 a}(44.8 \mathrm{mg}, 0.1 \mathrm{mmol})$ in anhydrous ether $(2.0 \mathrm{~mL})$ was added $\mathrm{PhMgBr}(0.3 \mathrm{mmol})$, under $\mathrm{N}_{2}$. The resulting mixture was stirred at r.t. for $5 \mathrm{~h}$ (monitored by TLC). Work-up: The mixture was poured into $\mathrm{NH}_{4} \mathrm{Cl}$ aqueous solution and extracted with DCM three times. The combined organic phases were dried over anhydrous $\mathrm{MgSO}_{4}$, filtered and concentrated under reduced pressure. The crude product was purified by silica gel flash chromatography and eluted by hexanes/EtOAc=20:1 then 10:1 to afford product 7 in $60 \%$ yield $(30.2 \mathrm{mg})$ as a yellow solid. ${ }^{1} \mathbf{H}$ NMR (500 MHz, $\left.\mathbf{C D C l}_{3}\right): \delta 7.30-7.05(\mathrm{~m}, 21 \mathrm{H}), 6.98(\mathrm{~d}, J=7.6 \mathrm{~Hz}$, 2H), $6.92(\mathrm{~d}, J=6.9 \mathrm{~Hz}, 2 \mathrm{H}), 6.02(\mathrm{~d}, J=10.1 \mathrm{~Hz}, 2 \mathrm{H}), 5.85(\mathrm{~d}, J=10.1 \mathrm{~Hz}, 2 \mathrm{H})$. ${ }^{13}$ C NMR (125 MHz, $\left.\mathbf{C D C l}_{3}\right): \delta$ 147.6, 146.6, 145.2, 145.1, 144.8, 136.5, 136.4, $135.7,135.4,134.2,130.6,130.53,130.46,130.3,128.5,128.27,128.25,128.2$, $128.03,127.96,127.8,127.4,127.3,127.2,126.1,77.8,77.5,77.2,69.3,64.2$. HRMS (ESI): calcd for $\mathrm{C}_{40} \mathrm{H}_{31} \mathrm{O}[\mathrm{M}+\mathrm{H}]^{+}$527.2375, found 527.2370.

\section{General procedure for the preparation of compounds 5}

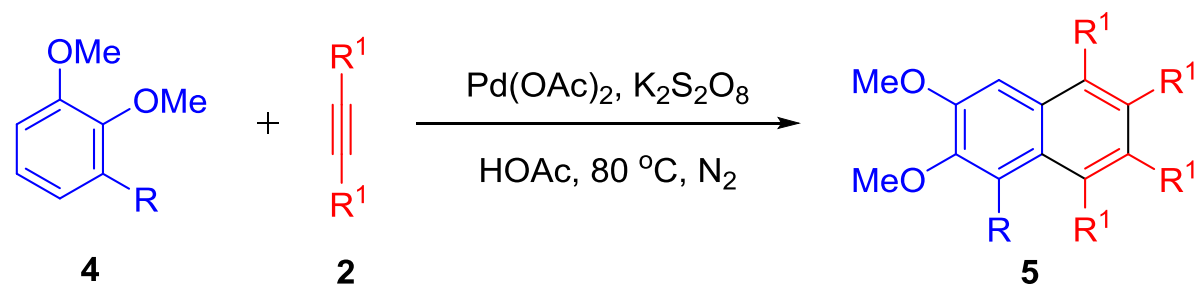

To a solution of alkyne $2(71.2 \mathrm{mg}, 0.2 \mathrm{mmol})$ in $4(1.0 \mathrm{~mL})$, palladium (II) acetate 
(4.5 mg, $10 \mathrm{~mol} \%)$, HOAc (12.0 uL, $0.2 \mathrm{mmol})$ and potassium persulfate $(108.0 \mathrm{mg}$, $0.4 \mathrm{mmol}$ ) were added. The reaction mixture was stirred at $80{ }^{\circ} \mathrm{C}$ in an oil bath under $\mathrm{N}_{2}$ until 2 was gone (10 h, monitored by TLC). Work-up: The reaction mixture was cooled down to room temperature. Solvent was removed under ScanVac CoolSafe Freeze Dryer, which was pre-adsorbed on silica gel and purified by flash chromatography (silica gel, EtOAc/hexanes) to afford pure product 5.

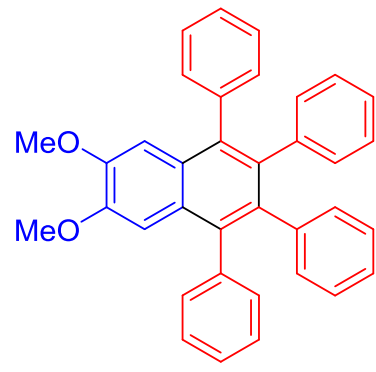

6,7-Dimethoxy-1,2,3,4-tetraphenylnaphthalene $(\mathbf{5 a}):{ }^{[4]}$ The title compound was prepared via the general procedure from 1,2-dimethoxybenzene $\mathbf{1 m}(2.0 \mathrm{~mL})$ and 1,2-diphenylethyne 2a (0.4 mmol, $71.3 \mathrm{mg})$. After purification by silica gel column chromatography (hexanes/EtOAc $=20: 1)$, the product was isolated in 92\% yield $(90.6$ mg) as a white solid. IR (KBr) / $\mathrm{cm}^{-1}:$ 3056, 2947, 1594, 1442, 1158, 745. ${ }^{\mathbf{1}} \mathbf{H}$ NMR (500 MHz, CDCl $\left.\mathbf{~}_{3}\right): \delta 7.35-7.14(\mathrm{~m}, 10 \mathrm{H}), 7.00(\mathrm{~s}, 2 \mathrm{H}), 6.93-6.78(\mathrm{~m}, 10 \mathrm{H}), 3.77(\mathrm{~s}$, 6H). ${ }^{13}$ C NMR (125 MHz, $\left.\mathbf{C D C l}_{3}\right): \delta$ 149.3, 140.8, 140.0, 137.4, 137.1, 131.5, 131.2, 127.8, 127.6, 126.5, 126.4, 125.2, 105.9, 77.3, 77.1, 76.8, 55.6. HRMS (EI): calcd for $\mathrm{C}_{36} \mathrm{H}_{28} \mathrm{O}_{2}[\mathrm{M}]^{+}$492.2089, found 492.2090.<smiles>COc1cc2c(-c3ccccc3)c(-c3ccccc3)c(-c3ccccc3)c(-c3ccccc3)c2c(OC)c1OC</smiles>

5,6,7-Trimethoxy-1,2,3,4-tetraphenylnaphthalene (5b): The title compound was prepared via the general procedure from 1,2,3-trimethoxybenzene 1n $(2.0 \mathrm{~mL})$ and 1,2-diphenylethyne 2a (0.4 mmol, $71.3 \mathrm{mg})$. After purification by silica gel column chromatography (hexanes/EtOAc $=20: 1)$, the product was isolated in $84 \%$ yield $(87.8$ $\mathrm{mg})$ as a white solid. IR (KBr) / $\mathrm{cm}^{-1}: 3021,2951,1620,1502,1465,1253,1128,814$. 
${ }^{1} \mathbf{H}$ NMR (500 MHz, $\left.\mathbf{C D C l}_{3}\right): \delta$ 7.28-7.17 (m, 7H), 7.15-7.10 (m, 2H), 7.08-7.03 (m, 1H), 6.88-6.77 (m, 11H), 3.90 (s, 3H), 3.72 (s, 3H), 3.29 (s, 3H). ${ }^{13}$ C NMR (125 MHz, $\left.\mathbf{C D C l}_{3}\right): \delta$ 152.6, 150.2, 143.1, 142.7, 140.9, 140.8, 140.7, 140.5, 140.2, 139.0, 138.9, 137.4, 136.0, 131.7, 131.6, 131.3, 131.2, 130.3, 130.2, 127.8, 126.7, 126.6, 126.33, 126.30, 125.32, 125.31, 125.29, 125.1, 125.0, 122.9, 102.7, 61.0, 60.8, 55.6. HRMS (EI): calcd for $\mathrm{C}_{37} \mathrm{H}_{30} \mathrm{O}_{3}[\mathrm{M}]^{+}$522.2195, found 522.2199.

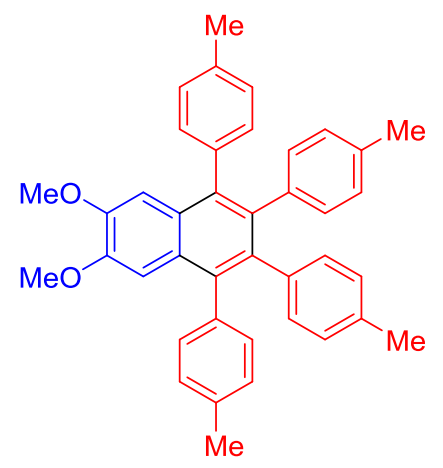

6,7-Dimethoxy-1,2,3,4-tetra-p-tolylnaphthalene (5c): The title compound was prepared via the general procedure from 1,2-dimethoxybenzene $\mathbf{1 m}(2.0 \mathrm{~mL})$ and 1,2-di-p-tolylethyne $\mathbf{2 b}(0.4 \mathrm{mmol}, 82.5 \mathrm{mg})$. After purification by silica gel column chromatography (hexanes/EtOAc $=20: 1$ ), the product was isolated in $95 \%$ yield $(104.3 \mathrm{mg})$ as a white solid. IR $(\mathrm{KBr}) / \mathrm{cm}^{-1}: 3021,2951,1620,1502,1465,1253$, 1128, 814. ${ }^{1} \mathbf{H}$ NMR (500 MHz, $\left.\mathbf{C D C l}_{3}\right): \delta 7.11(\mathrm{~d}, J=8.0 \mathrm{~Hz}, 4 \mathrm{H}), 7.07(\mathrm{~d}, J=7.9$ $\mathrm{Hz}, 4 \mathrm{H}), 6.98$ (s, 2H), 6.73 (d, J=8.0 Hz, 4H), $6.68(\mathrm{~d}, J=8.0 \mathrm{~Hz}, 4 \mathrm{H}), 3.76(\mathrm{~s}, 6 \mathrm{H})$, 2.34 (s, 6H), 2.12 (s, 6H). ${ }^{13} \mathbf{C}$ NMR (125 MHz, $\left.\mathbf{C D C l}_{3}\right): \delta 149.0,138.0,137.6,137.1$, 137.0, 135.5, 134.1, 131.3, 131.0, 128.3, 128.0, 127.2, 106.0, 77.3, 77.1, 76.8, 55.7, 21.3, 21.1. HRMS (EI): calcd for $\mathrm{C}_{40} \mathrm{H}_{36} \mathrm{O}_{2}[\mathrm{M}]^{+}$548.2715, found 548.2713.<smiles>COc1cc2c(-c3ccc(F)cc3)c(-c3ccc(F)cc3)c(-c3ccc(F)cc3)c(-c3ccc(F)cc3)c2cc1OC</smiles>

1,2,3,4-Tetrakis(4-fluorophenyl)-6,7-dimethoxynaphthalene (5d): The title 
compound was prepared via the general procedure from 1,2-dimethoxybenzene $1 \mathrm{~m}$ $(2.0 \mathrm{~mL})$ and 1,2-bis(4-fluorophenyl)ethyne $2 \mathbf{f}(0.4 \mathrm{mmol}, 85.7 \mathrm{mg})$. After purification by silica gel column chromatography (hexanes/EtOAc $=20: 1$ ), the product was isolated in $90 \%$ yield $(101.6 \mathrm{mg})$ as a white solid. IR $(\mathrm{KBr}) / \mathrm{cm}^{-1}: 3044$, 2954,1603, 1501, 1465, 1254, 1220, 1127, 832. ${ }^{1} \mathbf{H}$ NMR (500 MHz, $\left.\mathbf{C D C l}_{3}\right): \delta$ 7.21-7.11 (m, 4H), 7.04-6.96 (m, 4H), $6.90(\mathrm{~s}, 2 \mathrm{H}), 6.81-6.72(\mathrm{~m}, 4 \mathrm{H}), 6.67-6.59(\mathrm{~m}$, 4H), 3.78 (s, 6H). ${ }^{13} \mathbf{C}$ NMR (125 MHz, $\left.\mathbf{C D C l}_{3}\right): \delta 162.5,161.7,160.6,159.7,149.6$, $136.6,136.5,136.43,136.40,135.6,135.5,132.7,132.6,132.54,132.48,128.0,115.0$, 114.8, 114.0, 113.8, 105.5, 77.3, 77.0, 76.8, 55.7. HRMS (EI): calcd for $\mathrm{C}_{36} \mathrm{H}_{24} \mathrm{O}_{2} \mathrm{~F}_{4}$ $[\mathrm{M}]^{+}$564.1712, found 564.1703.

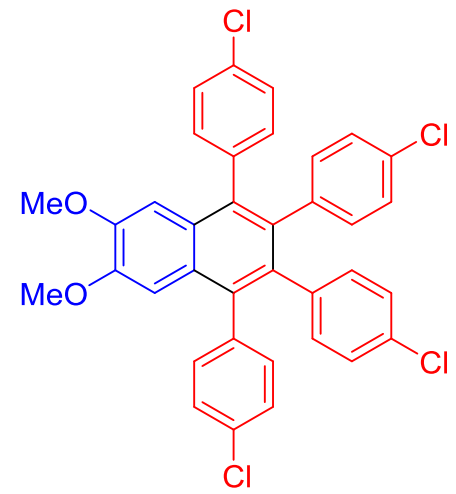

1,2,3,4-Tetrakis(4-chlorophenyl)-6,7-dimethoxynaphthalene (5e): The title compound was prepared via the general procedure from 1,2-dimethoxybenzene $\mathbf{1 m}$ $(2.0 \mathrm{~mL})$ and 1,2-bis(4-chlorophenyl)ethyne $\mathbf{2 g}(0.4 \mathrm{mmol}, 98.8 \mathrm{mg})$. After purification by silica gel column chromatography (hexanes/EtOAc $=20: 1$ ), the product was isolated in $85 \%$ yield $(107.2 \mathrm{mg})$ as a white solid. ${ }^{\mathbf{1}} \mathbf{H}$ NMR (500 $\mathbf{~ M H z}$, $\left.\mathbf{C D C l}_{3}\right): \delta 7.28(\mathrm{~d}, J=8.3 \mathrm{~Hz}, 4 \mathrm{H}), 7.14(\mathrm{~d}, J=8.3 \mathrm{~Hz}, 4 \mathrm{H}), 6.92(\mathrm{~d}, J=8.3 \mathrm{~Hz}, 4 \mathrm{H})$, $6.87(\mathrm{~s}, 2 \mathrm{H}), 6.74(\mathrm{~d}, J=8.4 \mathrm{~Hz}, 4 \mathrm{H}), 3.78(\mathrm{~s}, 6 \mathrm{H}) .{ }^{13} \mathbf{C}$ NMR (125 MHz, CDCl $\left.\mathbf{3}\right): \delta$ $149.8,138.6,137.9,136.3,135.9,132.8,132.4,132.3,131.7,128.2,127.8,127.3$, 105.5, 77.3, 77.0, 76.8, 55.8. HRMS (EI): calcd for $\mathrm{C}_{36} \mathrm{H}_{24} \mathrm{O}_{2} \mathrm{Cl}_{4}[\mathrm{M}]^{+}$628.0530, found 628.0523 . 


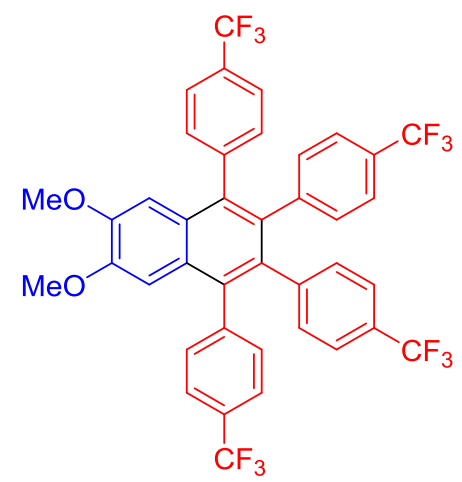

6,7-Dimethoxy-1,2,3,4-tetrakis(4-(trifluoromethyl)phenyl)naphthalene (5f): The title compound was prepared via the general procedure from 1,2-dimethoxybenzene $1 \mathbf{m}(2.0 \mathrm{~mL})$ and 1,2-bis(4-(trifluoromethyl)phenyl)ethyne $\mathbf{2 h}(0.4 \mathrm{mmol}, 125.7 \mathrm{mg})$. After purification by silica gel column chromatography (hexanes/EtOAc $=20: 1$ ), the product was isolated in $70 \%$ yield $\left(107.0 \mathrm{mg}\right.$ ) as a white solid. ${ }^{\mathbf{1}} \mathbf{H}$ NMR (500 $\mathbf{~ M H z}$, $\left.\mathbf{C D C l}_{3}\right): \delta 7.58(\mathrm{~d}, J=8.1 \mathrm{~Hz}, 4 \mathrm{H}), 7.36(\mathrm{~d}, J=8.0 \mathrm{~Hz}, 4 \mathrm{H}), 7.19(\mathrm{~d}, J=8.2 \mathrm{~Hz}, 4 \mathrm{H})$, $6.95(\mathrm{~d}, J=8.1 \mathrm{~Hz}, 4 \mathrm{H}), 6.84(\mathrm{~s}, 2 \mathrm{H}), 3.78(\mathrm{~s}, 6 \mathrm{H}) .{ }^{13} \mathbf{C}$ NMR (125 MHz, $\left.\mathbf{C D C l}_{3}\right): \delta$ $150.3,143.5,142.9,136.3,135.4,131.4,131.3,129.4$ (q, $J=32.4 \mathrm{~Hz}), 128.3$ (q, $J=$ $32.7 \mathrm{~Hz}), 127.7,125.0(\mathrm{q}, J=3.5 \mathrm{~Hz}), 124.02(\mathrm{q}, J=3.4 \mathrm{~Hz}), 124.01(\mathrm{q}, J=272.1$ Hz) $, 123.8(\mathrm{q}, J=271.9 \mathrm{~Hz}), 105.3,77.3,77.0,76.8,55.8$. HRMS (EI): calcd for $\mathrm{C}_{40} \mathrm{H}_{24} \mathrm{O}_{2} \mathrm{~F}_{12}[\mathrm{M}]^{+}$764.1585, found 764.1595.

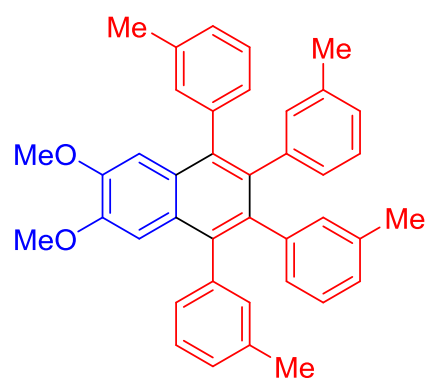

6,7-Dimethoxy-1,2,3,4-tetra-m-tolylnaphthalene (5g): The title compound was prepared via the general procedure from 1,2-dimethoxybenzene $\mathbf{1 m}(2.0 \mathrm{~mL})$ and 1,2-di- $m$-tolylethyne $2 \mathbf{d}(0.4 \mathrm{mmol}, 82.5 \mathrm{mg})$. After purification by silica gel column chromatography (hexanes/EtOAc $=20: 1$ ), the product was isolated as 2 inseparable regioisomers (ratio: $1: 14)$ in $89 \%$ yield $\left(97.7 \mathrm{mg}\right.$ ) as a white solid. ${ }^{\mathbf{1}} \mathbf{H}$ NMR (500 MHz, $\left.\mathbf{C D C l}_{3}\right): \delta 7.21-7.12(\mathrm{~m}, 2 \mathrm{H}), 7.11-6.92(\mathrm{~m}, 8 \mathrm{H}), 6.82-6.57(\mathrm{~m}, 8 \mathrm{H})$, 3.94-3.73 (m, 6H), 2.43-1.87 (m, 12H). ${ }^{13} \mathbf{C}$ NMR (125 MHz, $\left.\mathbf{C D C l}_{3}\right): \delta$ 149.0, $140.7,139.9,137.53,137.51,136.9,136.8,135.52,135.45,135.4,132.53,132.51$, 
$132.4,132.3,132.10,132.06,132.0,128.60,128.58,128.5,128.4,128.3,128.2,128.1$, $127.7,127.3,126.9,126.2,126.1,126.0,125.7,120.9,111.4,106.0,77.3,77.1,76.8$, 55.8, 55.7, 21.3, 21.1. HRMS (EI): calcd for $\mathrm{C}_{40} \mathrm{H}_{36} \mathrm{O}_{2}[\mathrm{M}]^{+}$548.2715, found 548.2717 .

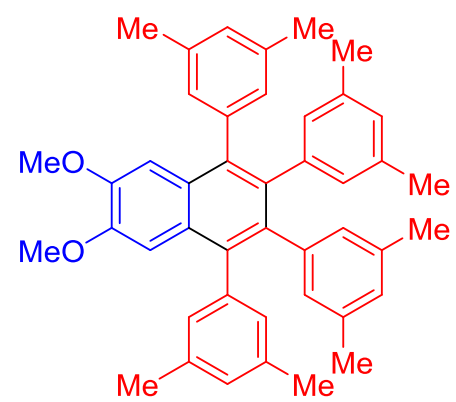

1,2,3,4-Tetrakis(3,5-dimethylphenyl)-6,7-dimethoxynaphthalene (5h): The title compound was prepared via the general procedure from 1,2-dimethoxybenzene $1 \mathbf{m}$ (2.0 $\mathrm{mL})$ and 1,2-bis(3,5-dimethylphenyl)ethyne 2e $(0.4 \mathrm{mmol}, 93.7 \mathrm{mg})$. After purification by silica gel column chromatography (hexanes/EtOAc $=20: 1$ ), the product was isolated in $88 \%$ yield $(106.5 \mathrm{mg})$ as a white solid. IR $(\mathrm{KBr}) / \mathrm{cm}^{-1}: 3001$, 2954, 1593, 1506, 1441, 754. ${ }^{\mathbf{1}} \mathbf{H}$ NMR (500 MHz, $\left.\mathbf{C D C l}_{3}\right): \delta 6.98$ (s, 2H), 6.85 (s, $4 \mathrm{H}), 6.80(\mathrm{~s}, 2 \mathrm{H}), 6.48(\mathrm{~s}, 4 \mathrm{H}), 6.44(\mathrm{~s}, 2 \mathrm{H}), 3.77(\mathrm{~s}, 6 \mathrm{H}), 2.24(\mathrm{~s}, 12 \mathrm{H}), 2.01(\mathrm{~s}, 12 \mathrm{H})$. ${ }^{13}$ C NMR (125 MHz, $\left.\mathbf{C D C l}_{3}\right): \delta$ 148.8, 140.6, 139.9, 137.7, 136.8, 136.4, 134.9, 129.4, 129.1, 127.6, 126.3, 106.1, 77.3, 77.0, 76.8, 55.7, 21.2, 21.0. HRMS (EI): calcd for $\mathrm{C}_{44} \mathrm{H}_{44} \mathrm{O}_{2}[\mathrm{M}]^{+}$604.3341, found 604.3340 .

\section{Proposed mechamism for the formation of compounds 5}

A plausible mechanism for functionalized naphthalenes synthesis is given above. The formation of naphthalenes starts with the palladation of $\mathbf{2 a}$ to yield the intermediate $\mathbf{I}$ or II. Then, vinyl-palladium intermediate $\mathbf{I V}^{\prime}$ is formed from intermediate III with the second alkyne unit inserted or intermediate II via a para-C-H functionalization process. At this point, a dienylpalladium intermediate $\mathbf{V}^{\prime}$ via orth-C-H activation or spirocyclic species $\mathbf{V I}^{\prime}$ is formed. Intermediate VI' undergoes a set of rearrangements to offer a 1,3-cyclohexadiene fused intermediate VII' due to possible increasing the electron density in benzene ring by the additional

OMe group. 5a is directly furnished from the former intermediate $\mathbf{V}^{\prime}$ or from the 
intermediate VII' via deprotonation together with the regeneration of palladium catalyst.
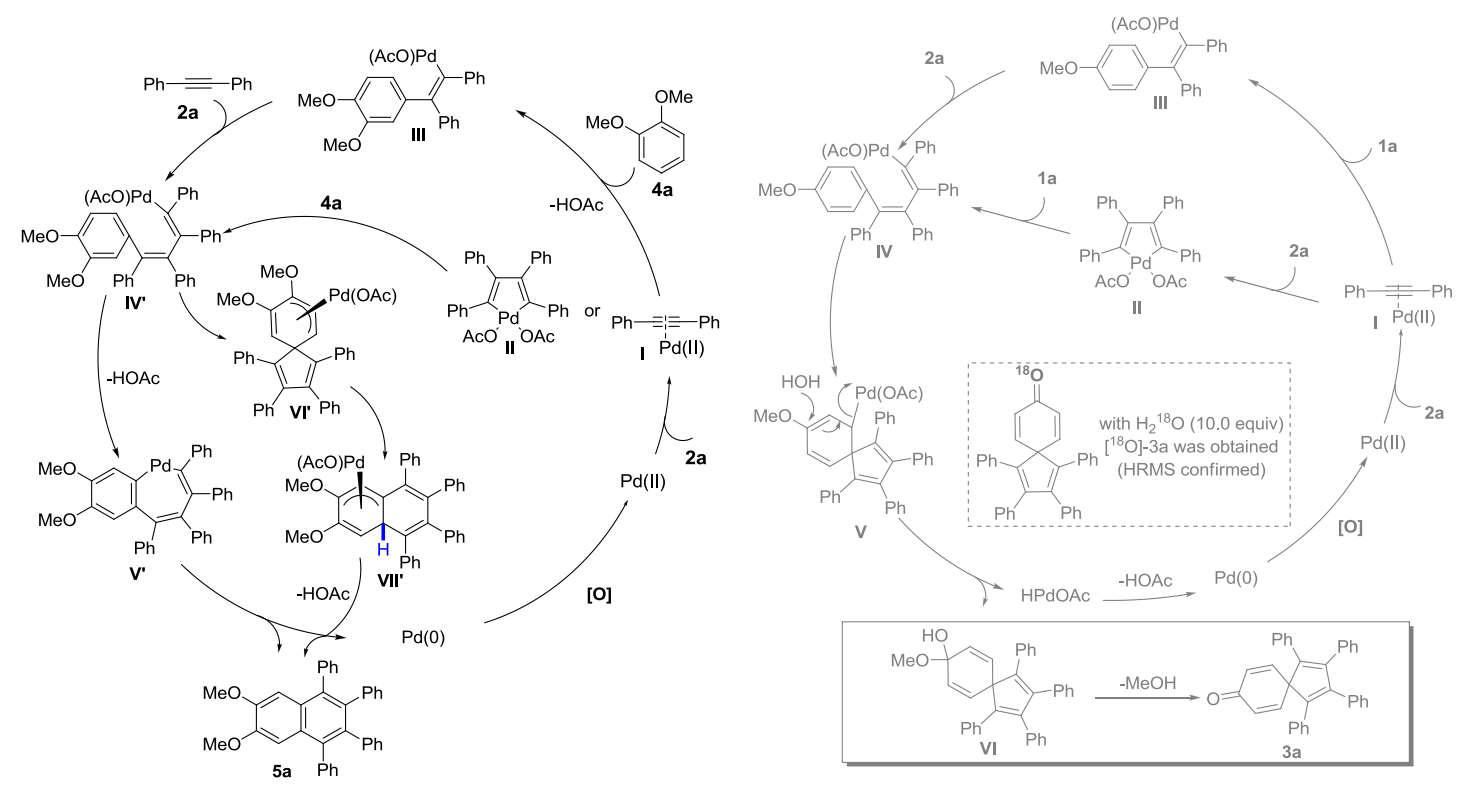

\section{X-ray analysis.}

X-Ray quality crystals for the compound 3a were prepared by interlayer diffusion of $n$-hexane into EtOAc solution within 2 days; for the compound $5 \mathbf{a}$ were prepared by slow evaporation of solvent from EtOAc solution within 3 days.

CCDC 1953883 and 1953747 contain the supplementary crystallographic data for 3a and 5a, respectively. These data can be obtained free of charge from The Cambridge Crystallographic Data Centre via https://www.ccdc.cam.ac.uk/structures.

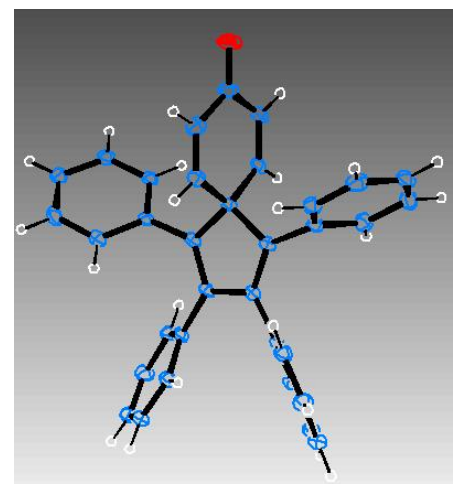

The ORTEP Drawing of 3a (The ellipsoid contour 30\% probability levels)

\section{Table S1. Crystal data and structure refinement for 3a.}


Empirical formula

Formula weight

Temperature

Wavelength

Crystal system

Space group

Unit cell dimensions

Volume

Z

Density (calculated)

Absorption coefficient

$\mathrm{F}(000)$

Crystal size

Theta range for data collection

Index ranges

Reflections collected

Independent reflections

Completeness to theta $=27.50^{\circ}$

Absorption correction

Max. and min. transmission

Refinement method

Data / restraints / parameters

Goodness-of-fit on $\mathrm{F}^{2}$

Final $\mathrm{R}$ indices [I $>2 \operatorname{sigma}(\mathrm{I})]$

$\mathrm{R}$ indices (all data)

Largest diff. peak and hole
C34 H24 O

448.53

100(2) K

$0.71073 \AA$

Monoclinic

$\mathrm{P} 2(1) / \mathrm{n}$

$\begin{array}{ll}\mathrm{a}=8.7972(10) \AA & \mathrm{a}=90^{\circ} . \\ \mathrm{b}=10.8213(12) \AA & \mathrm{b}=95.483(3)^{\circ} . \\ \mathrm{c}=25.050(3) \AA & \mathrm{g}=90^{\circ} .\end{array}$

2373.8(5) $\AA^{3}$

4

$1.255 \mathrm{Mg} / \mathrm{m}^{3}$

$0.074 \mathrm{~mm}^{-1}$

944

$0.30 \times 0.25 \times 0.20 \mathrm{~mm}^{3}$

2.05 to $27.50^{\circ}$.

$-11<=\mathrm{h}<=11,-14<=\mathrm{k}<=11,-31<=\mathrm{l}<=32$

16530

$5435[\mathrm{R}(\mathrm{int})=0.0677]$

$99.8 \%$

Semi-empirical from equivalents

0.9854 and 0.9782

Full-matrix least-squares on $\mathrm{F}^{2}$

5435 / 0 / 316

1.094

$\mathrm{R} 1=0.0789, \mathrm{wR} 2=0.1666$

$\mathrm{R} 1=0.1222, \mathrm{wR} 2=0.1837$

0.395 and -0.232 e. $\AA^{-3}$ 


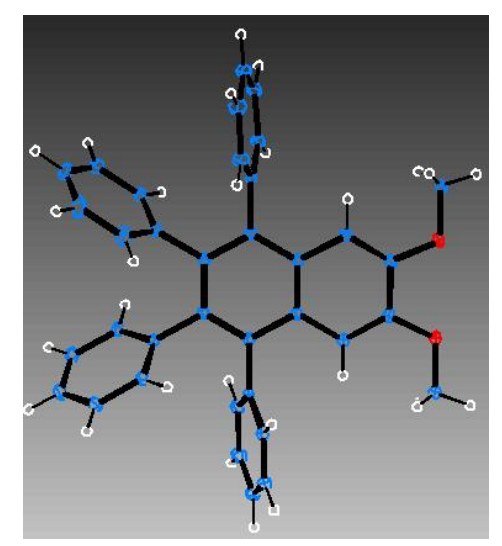

The ORTEP Drawing of 5a (The ellipsoid contour 30\% probability levels)

Table S2. Crystal data and structure refinement for 5a.

Identification code

Empirical formula

Formula weight

Temperature

Wavelength

Crystal system

Space group

Unit cell dimensions

Volume

$\mathrm{Z}$

Density (calculated)

Absorption coefficient

$\mathrm{F}(000)$

Crystal size

Theta range for data collection

Index ranges

Reflections collected

Independent reflections

Completeness to theta $=25.242^{\circ}$

Absorption correction

Max. and min. transmission

Refinement method

Data / restraints / parameters

Goodness-of-fit on $\mathrm{F}^{2}$
$5 \mathrm{a}$

$\mathrm{C} 36 \mathrm{H} 28 \mathrm{O} 2$

492.58

100(2) K

$0.71073 \AA$

Tetragonal

I $41 / \mathrm{a}$

$\mathrm{a}=23.7636(13) \AA$

$\mathrm{a}=90^{\circ}$.

$\mathrm{b}=23.7636(13) \AA$

$\mathrm{b}=90^{\circ}$.

$\mathrm{c}=18.7644(11) \AA$

$\mathrm{g}=90^{\circ}$.

10596.4(13) $\AA^{3}$

16

$1.235 \mathrm{Mg} / \mathrm{m}^{3}$

$0.075 \mathrm{~mm}^{-1}$

4160

$0.490 \times 0.440 \times 0.340 \mathrm{~mm}^{3}$

2.202 to $27.493^{\circ}$.

$-30<=\mathrm{h}<=30,-30<=\mathrm{k}<=30,-24<=\mathrm{l}<=24$

151067

$6080[\mathrm{R}($ int $)=0.0337]$

$100.0 \%$

Semi-empirical from equivalents

0.7457 and 0.7118

Full-matrix least-squares on $\mathrm{F}^{2}$

6080 / 0 / 345

1.038 
Final R indices [I $>2 \operatorname{sigma}(\mathrm{I})]$

$\mathrm{R}$ indices (all data)

Extinction coefficient

Largest diff. peak and hole
$\mathrm{R} 1=0.0405, \mathrm{wR} 2=0.1030$

$\mathrm{R} 1=0.0473, \mathrm{wR} 2=0.1082$

$\mathrm{n} / \mathrm{a}$

0.282 and -0.223 e. $\AA^{-3}$

\section{References}

[1] H. E. Gottlieb, V. Kotlyar, A. Nudelman, J. Org. Chem. 1997, 62, 7512.

[2] (a) P. L. Zhao, G. F. Yang, J. Am. Chem. Soc. 2010, 132, 185; (b) K. Park, S. Lee, J. Org. Chem. 2010, 75, 6244; (c) E. Shirakawa, T. Tsuchimoto, Tetrahedron 2005, 61, 9878.

[3] B. Schmidt, R. Berger, A. Kelling, U. Schilde, Chem. Eur. J. 2011, 17, 7032.

[4] Y. T. Wu, K. H. Huang, C. C. Shin, T. C. Wu, Chem. Eur. J. 2008, 14, 6697. 
13. ${ }^{1} \mathrm{H}$ NMR and ${ }^{13} \mathrm{C}$ NMR spectra
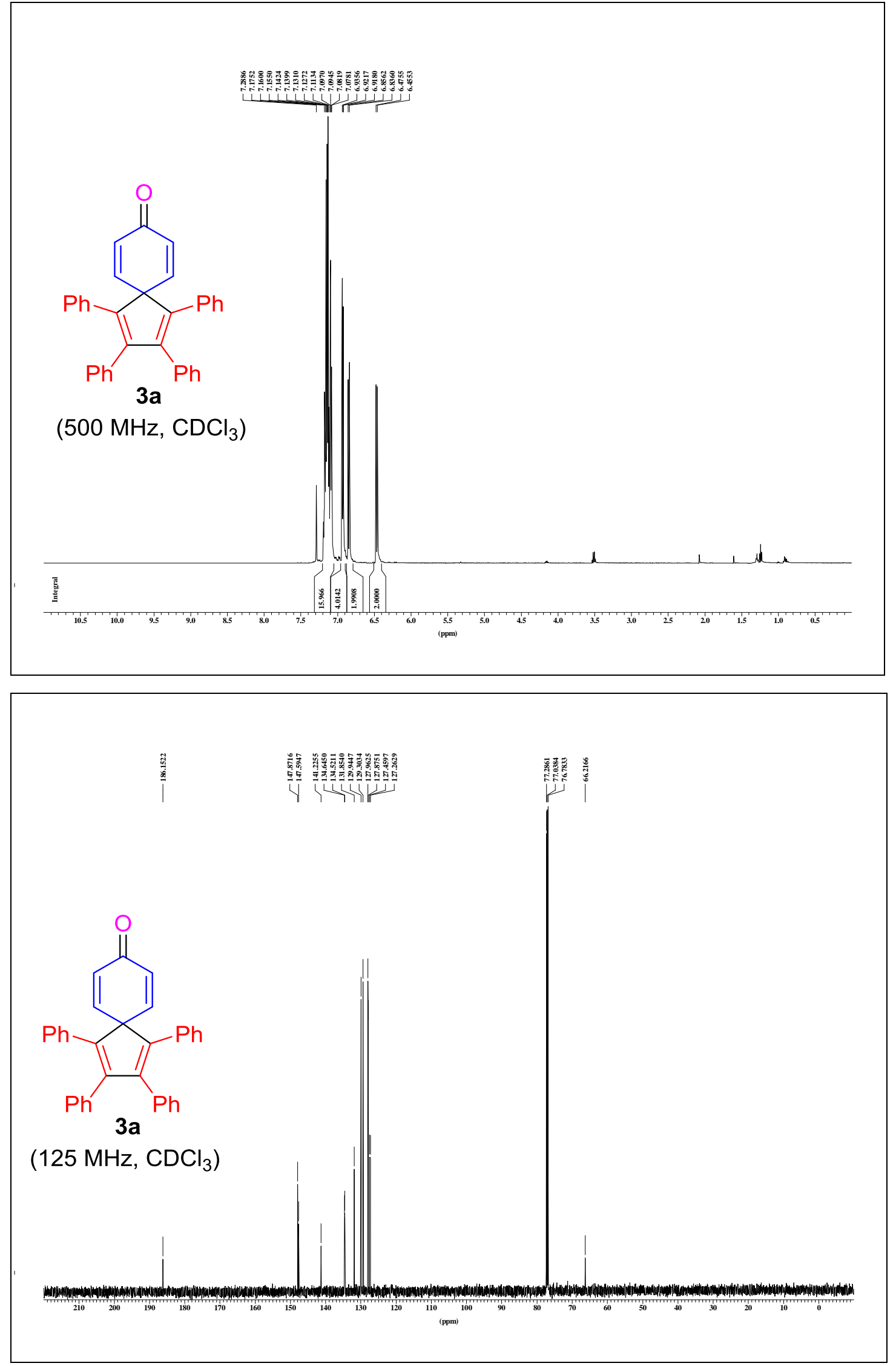


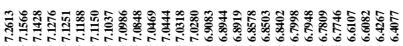<smiles>CC1=CC2(C=CC1=O)C(c1ccccc1)=C(c1ccccc1)C(c1ccccc1)=C2c1ccccc1</smiles>

(500 MHz, $\mathrm{CDCl}_{3}$ )
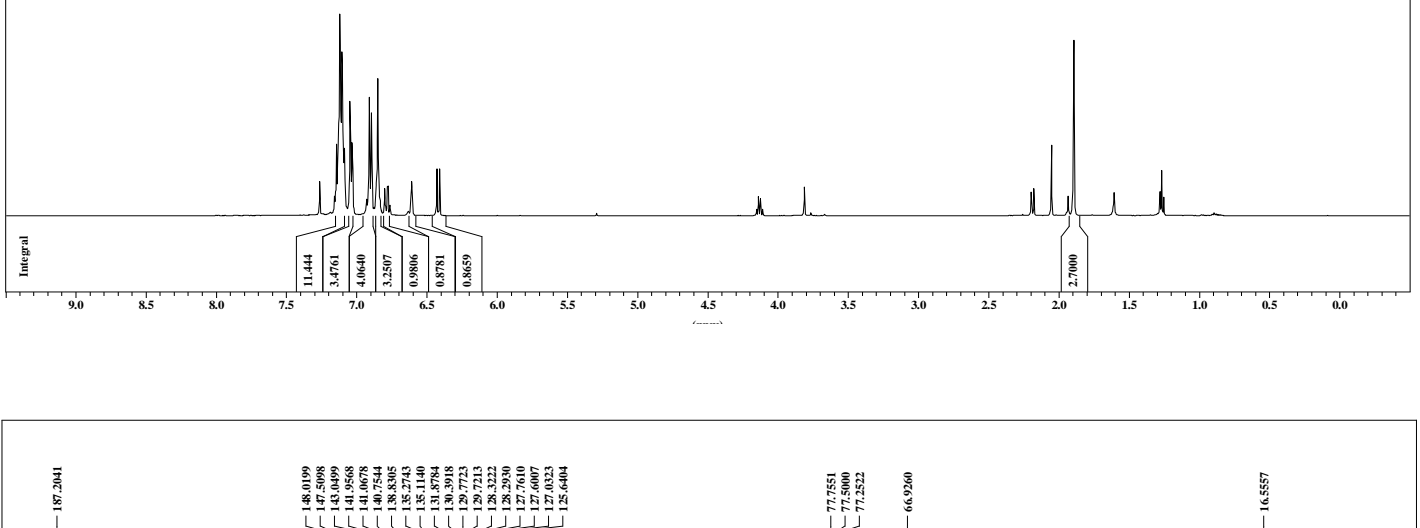<smiles>CC1=CC2(C=CC1=O)C(c1ccccc1)=C(c1ccccc1)C(c1ccccc1)=C2c1ccccc1</smiles>

3b

(125 MHz, $\mathrm{CDCl}_{3}$ ) 

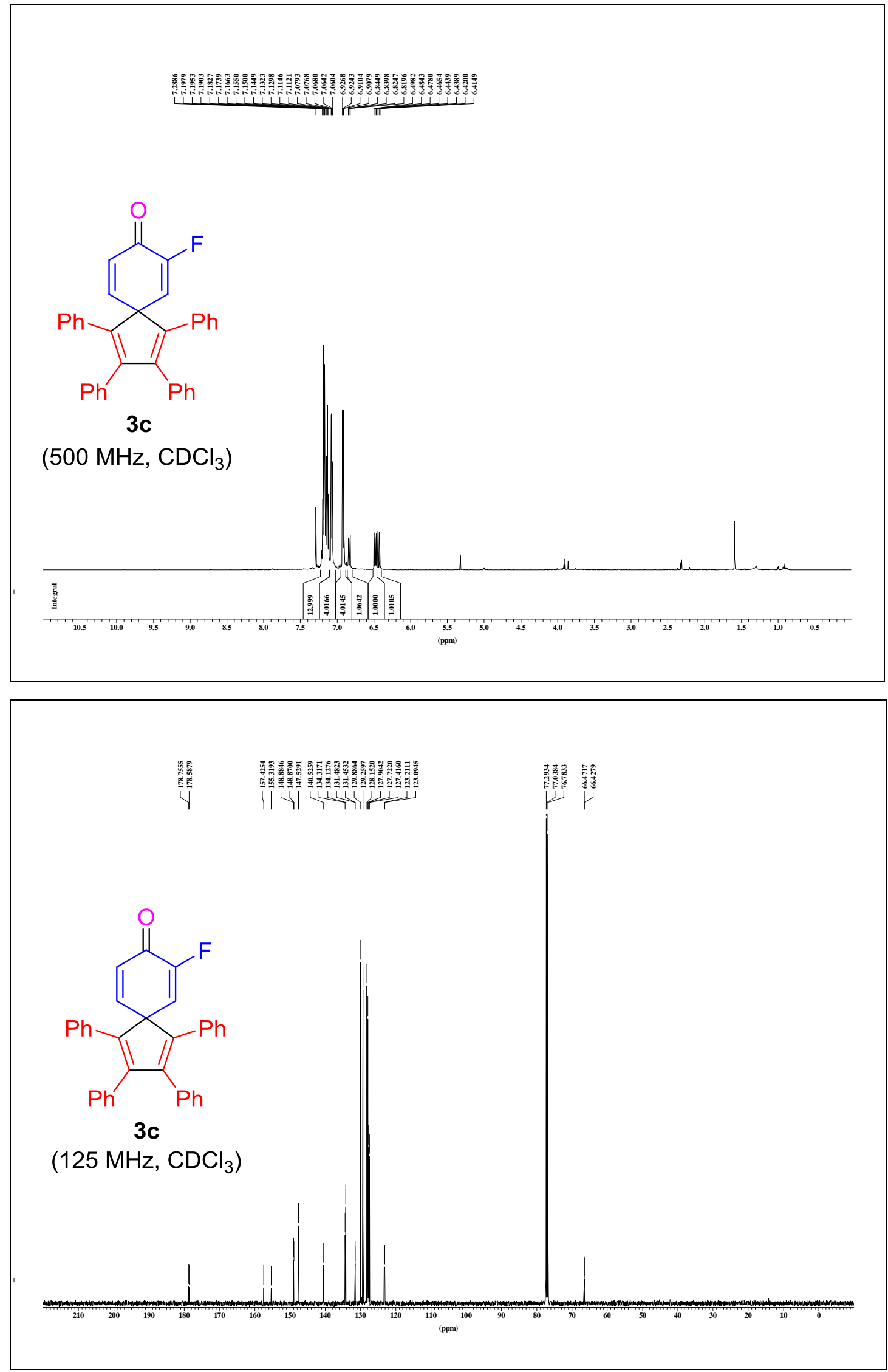

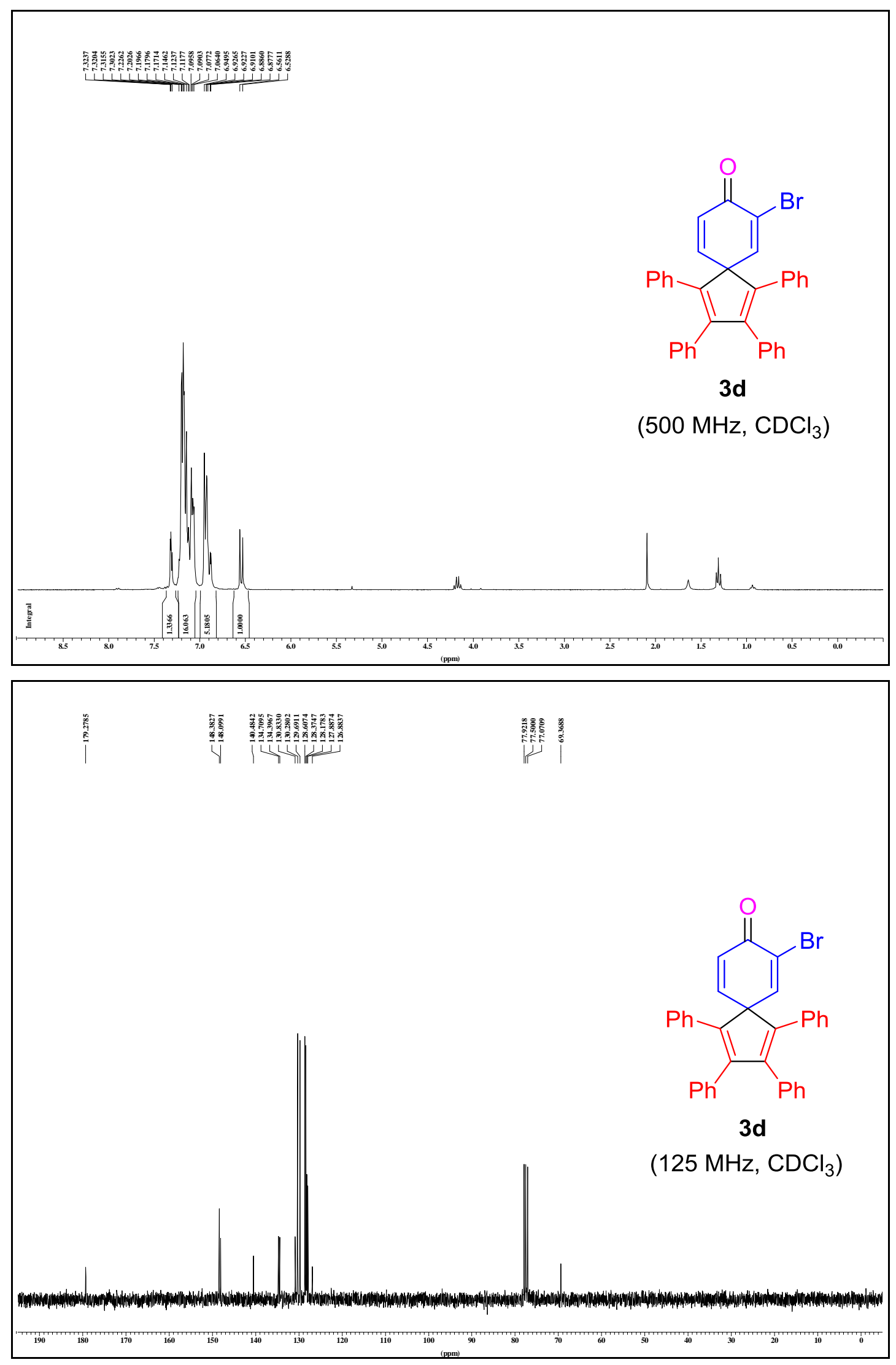


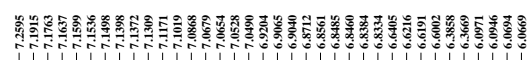<smiles>O=C1C=CC2(C(F)=C1)C(F)=C(c1ccccc1)C(c1ccccc1)=C2c1ccccc1</smiles>

$3 e$

$\left(500 \mathrm{MHz}, \mathrm{CDCl}_{3}\right.$ )
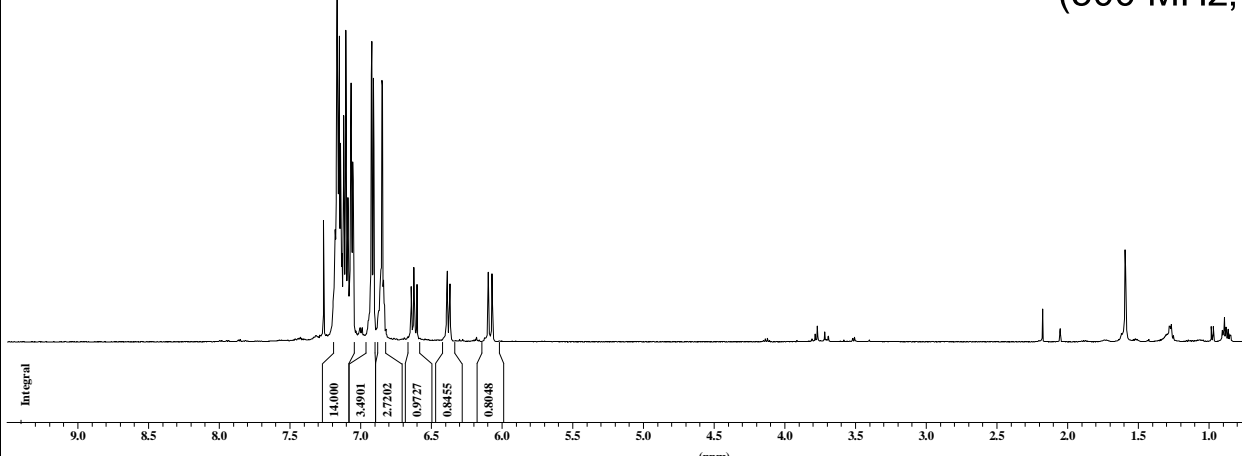

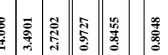
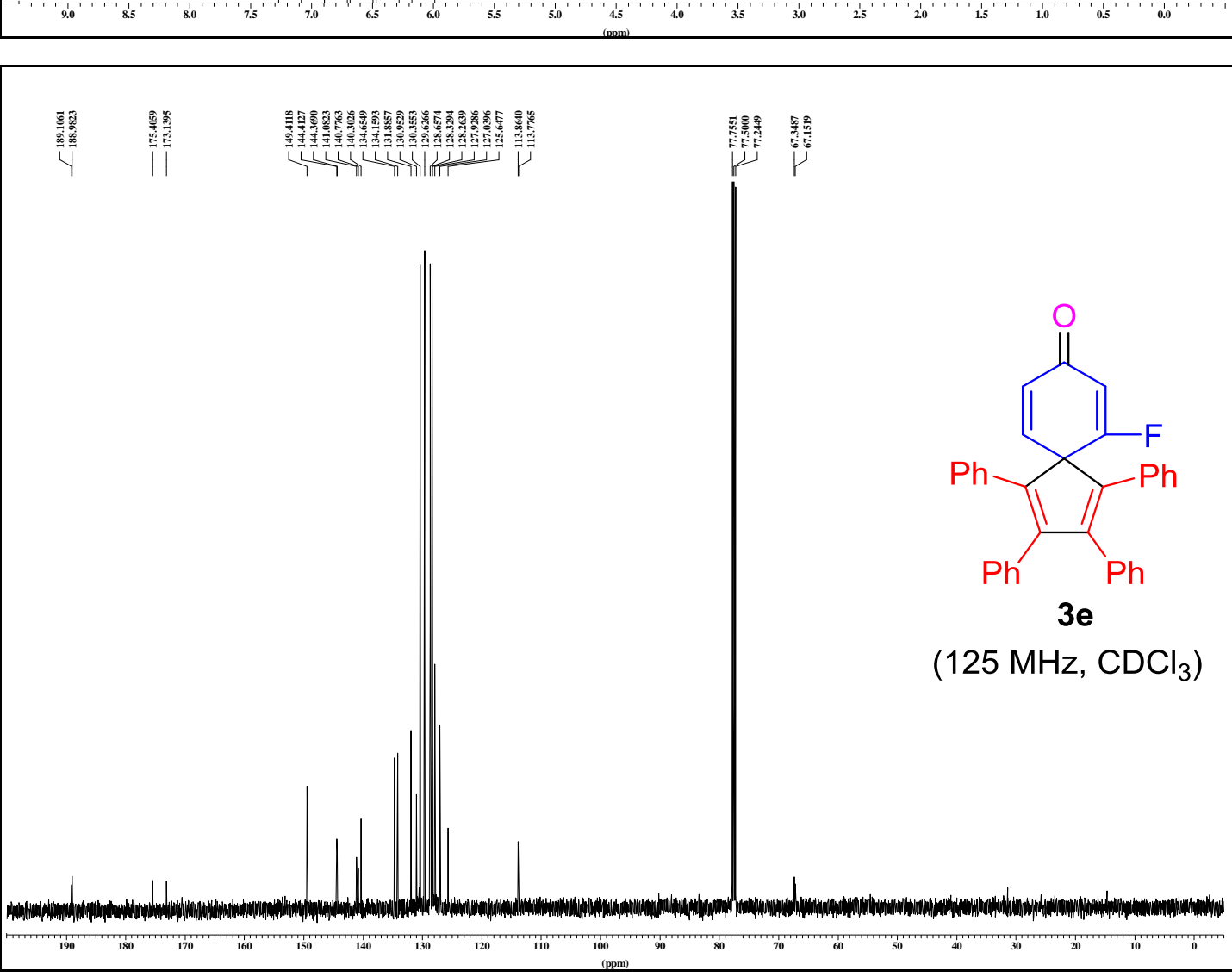


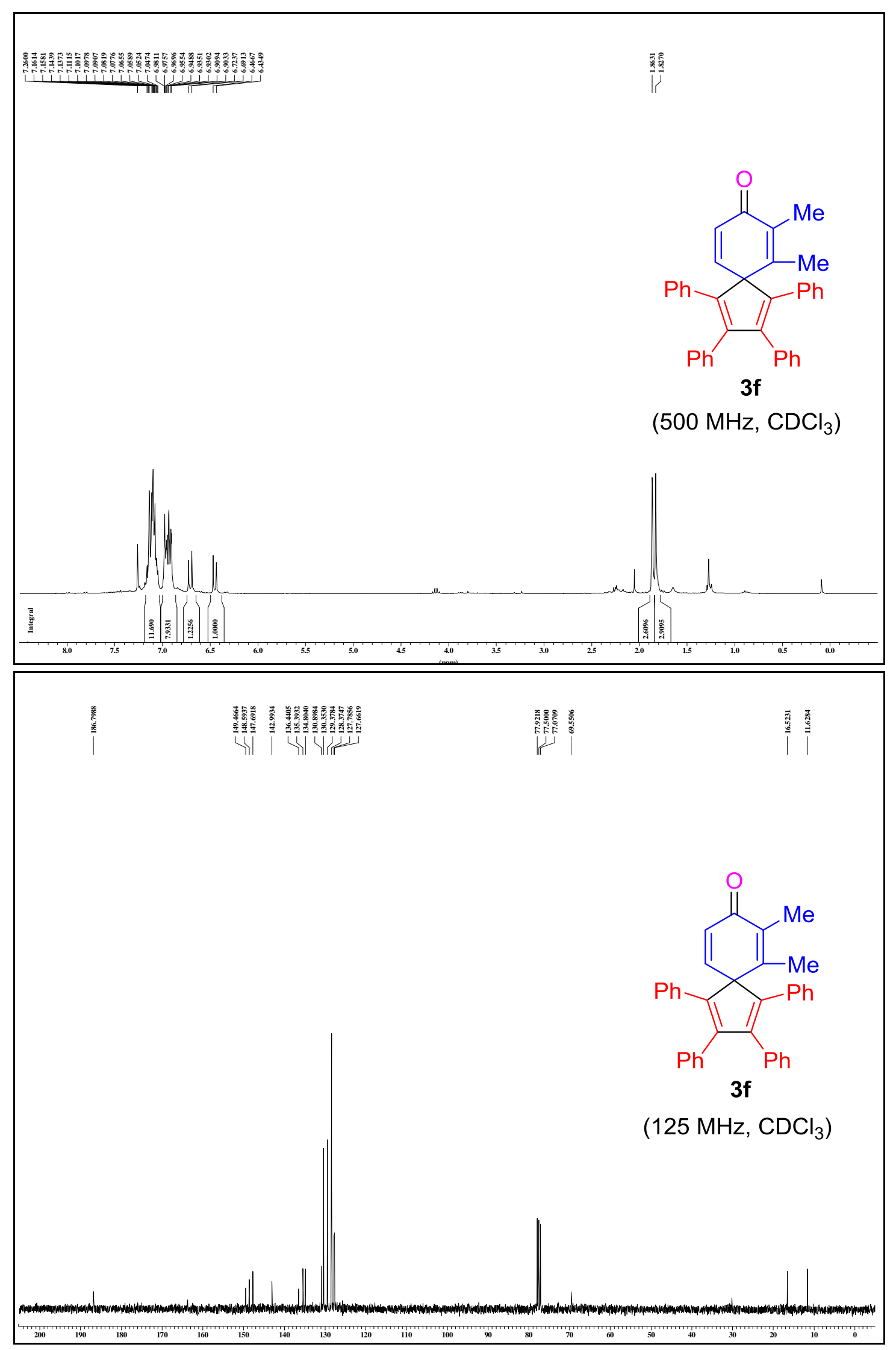



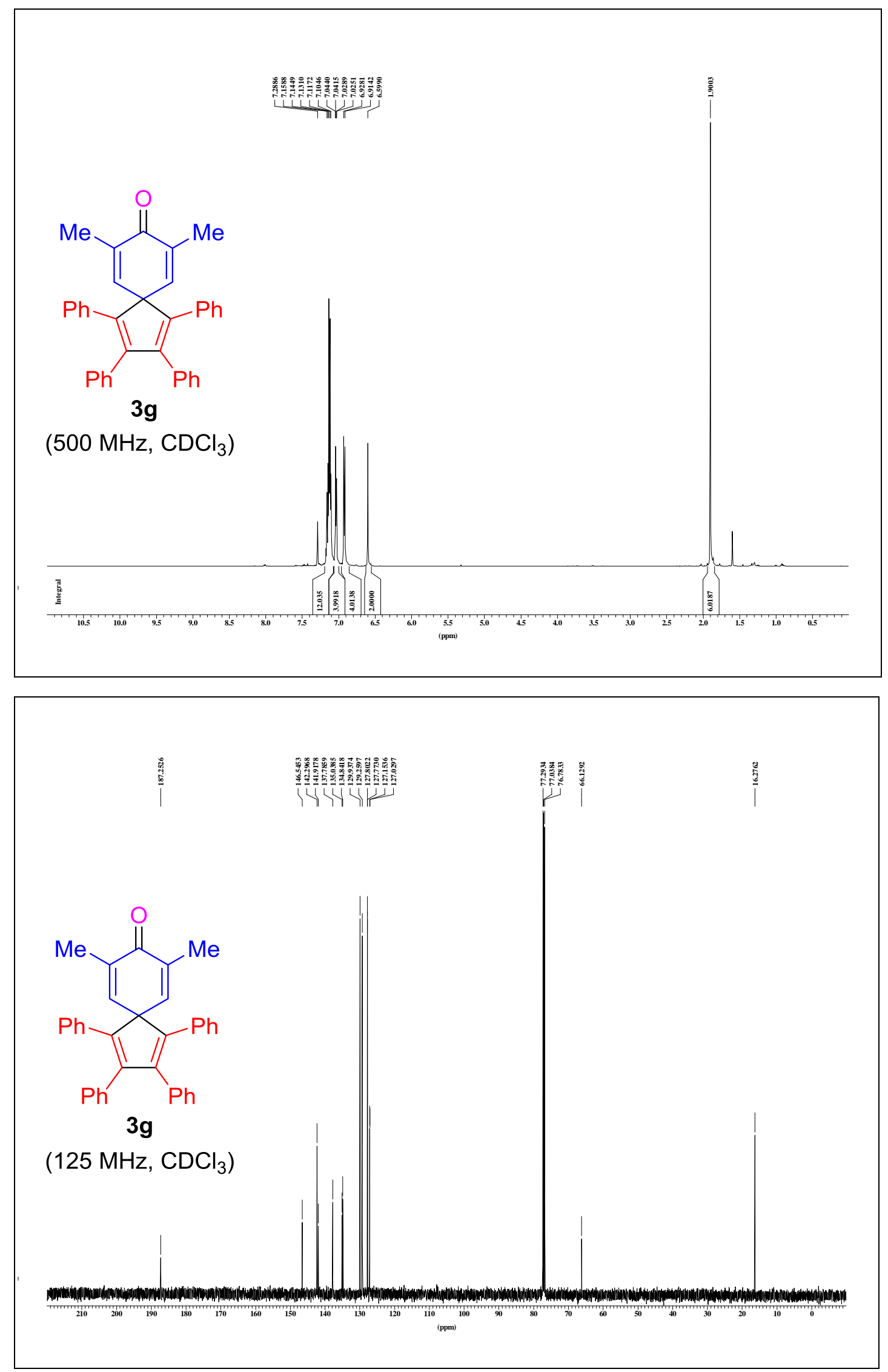

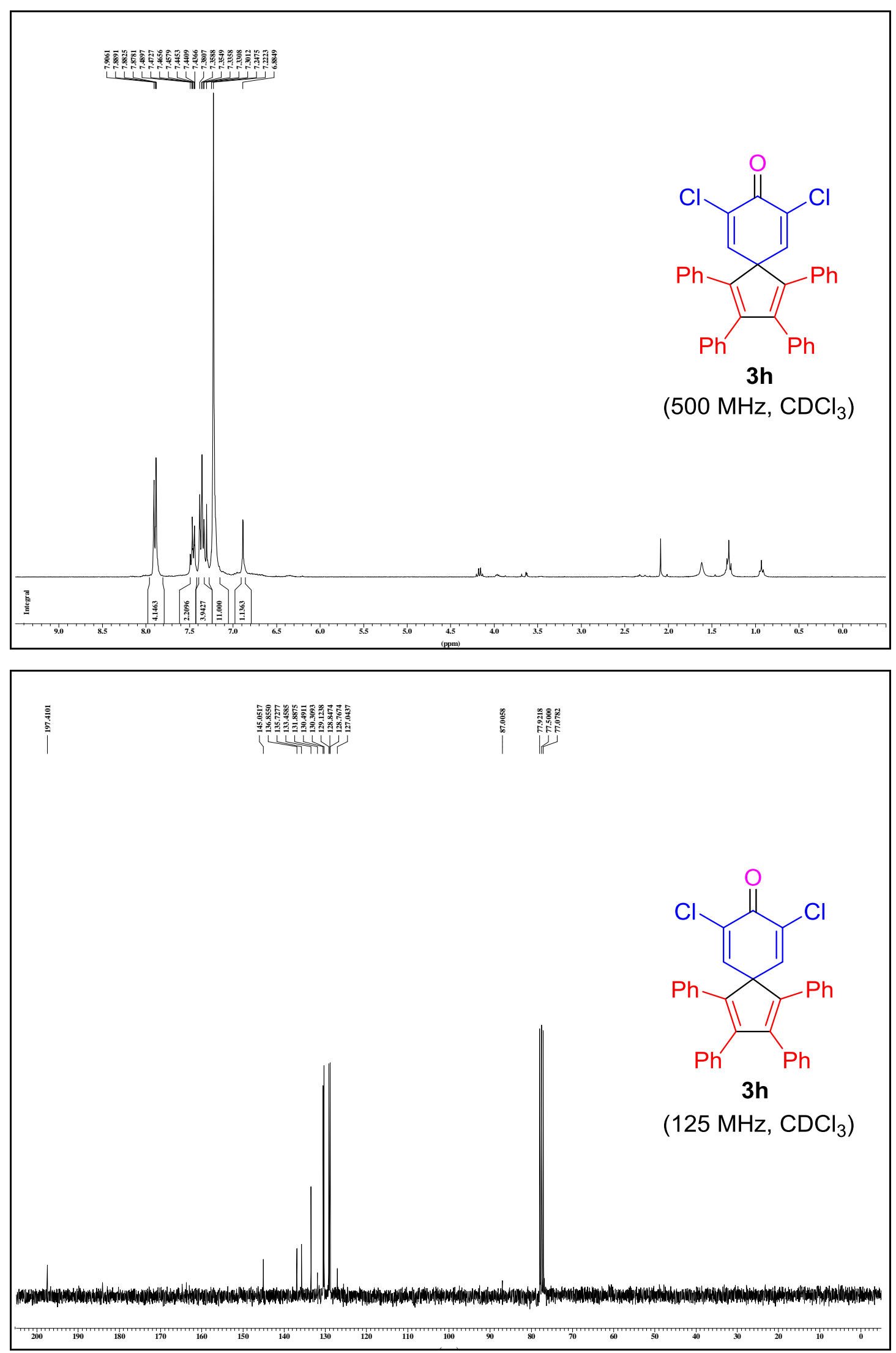


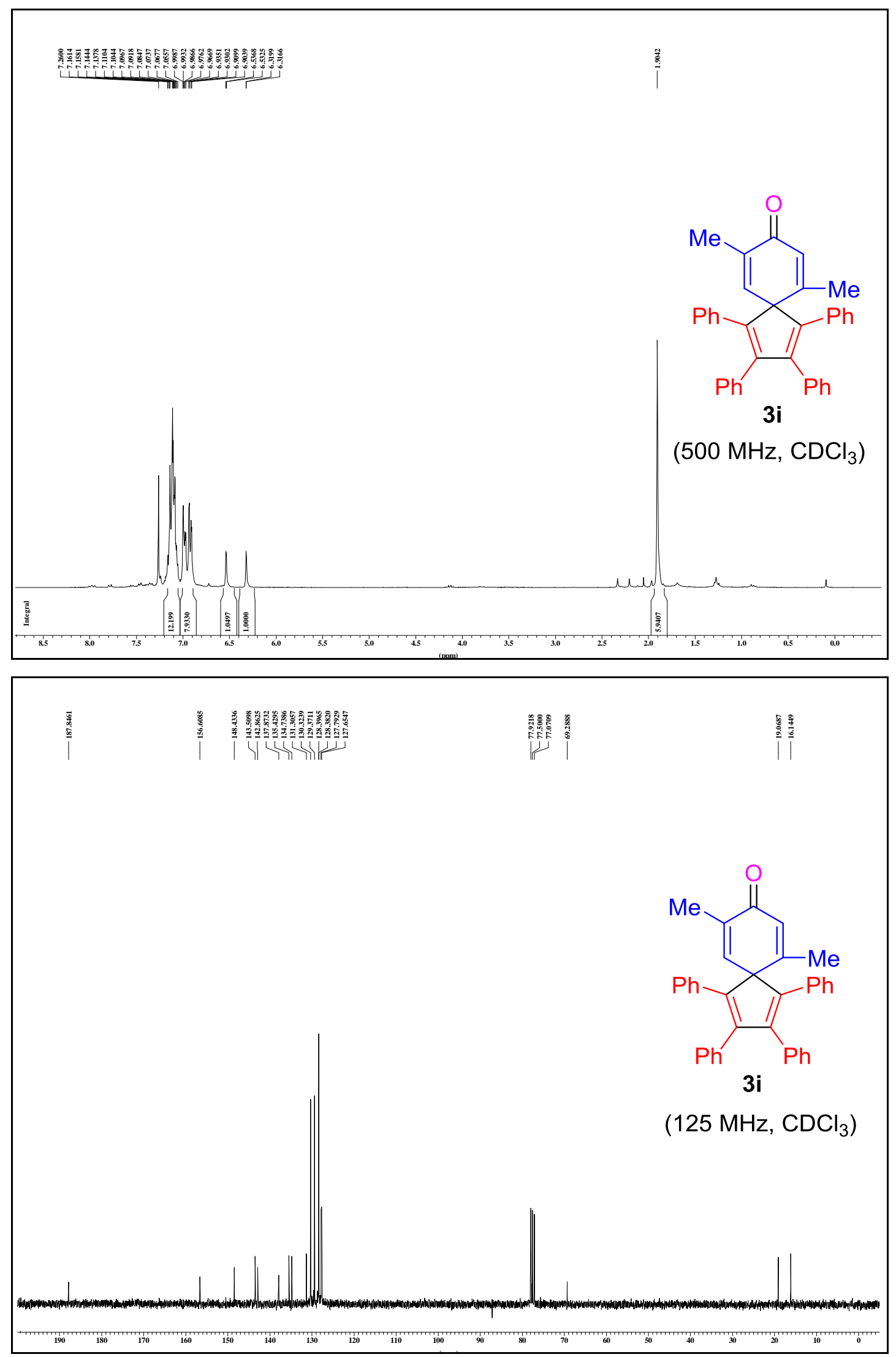



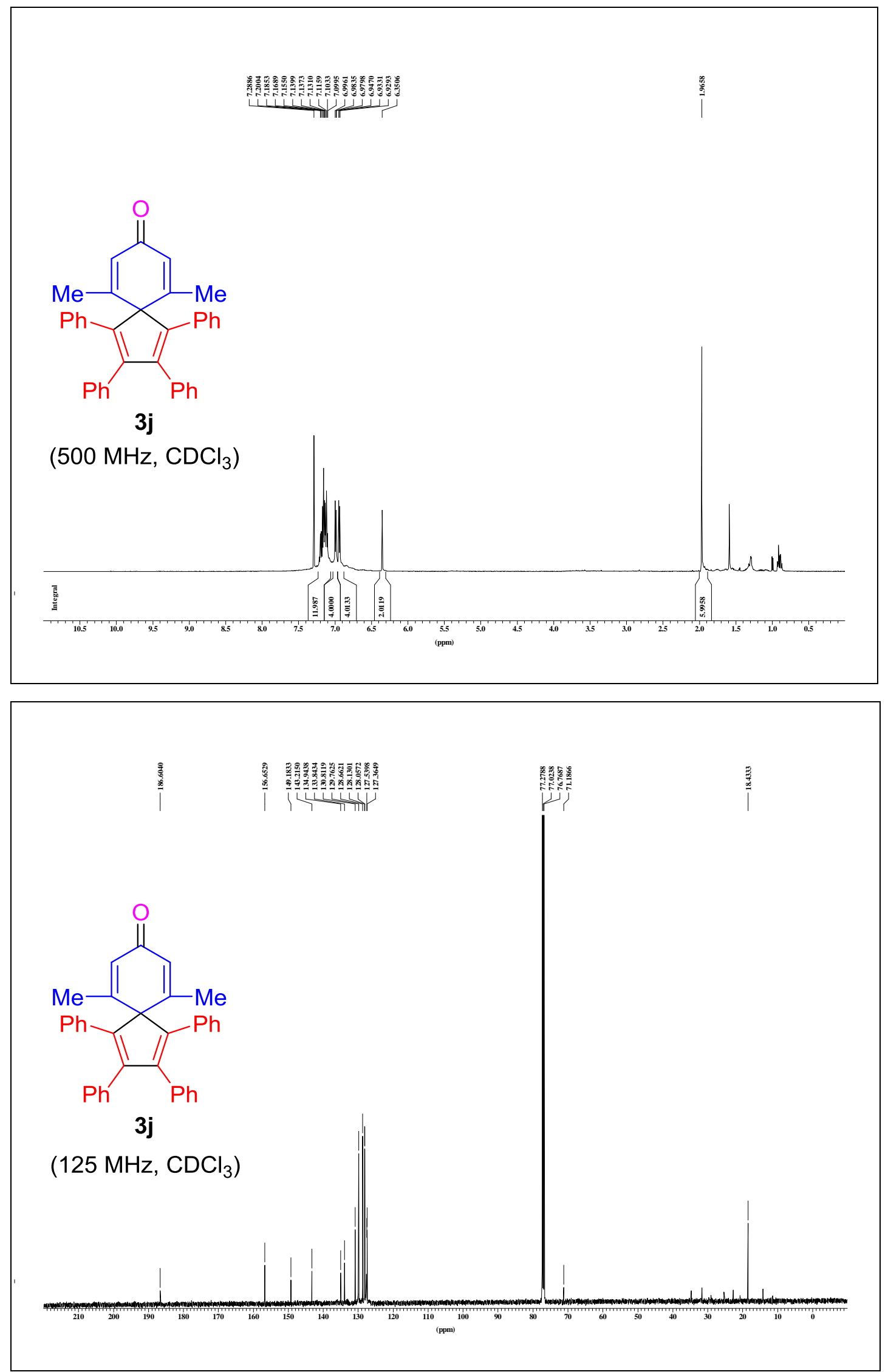


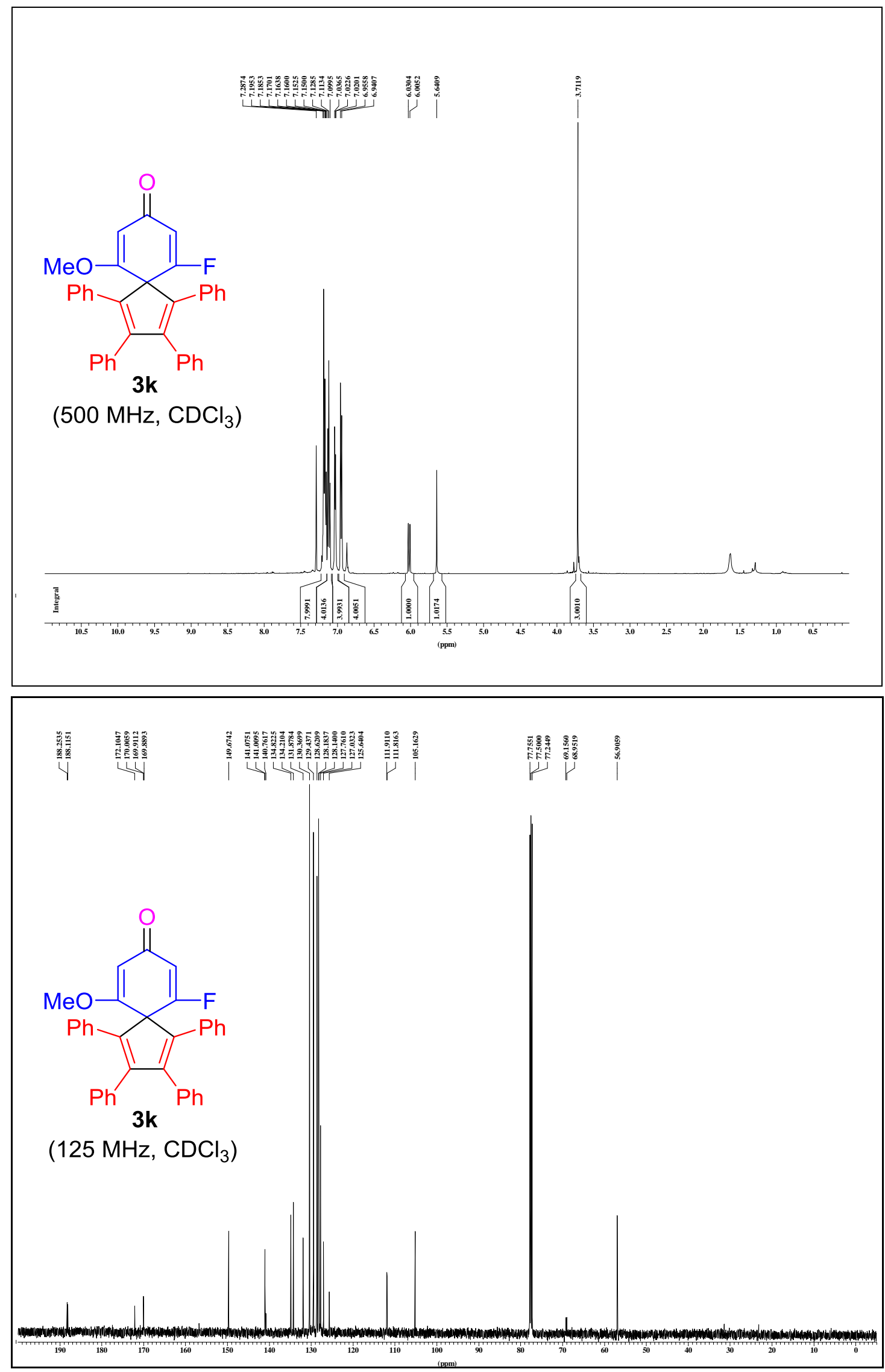




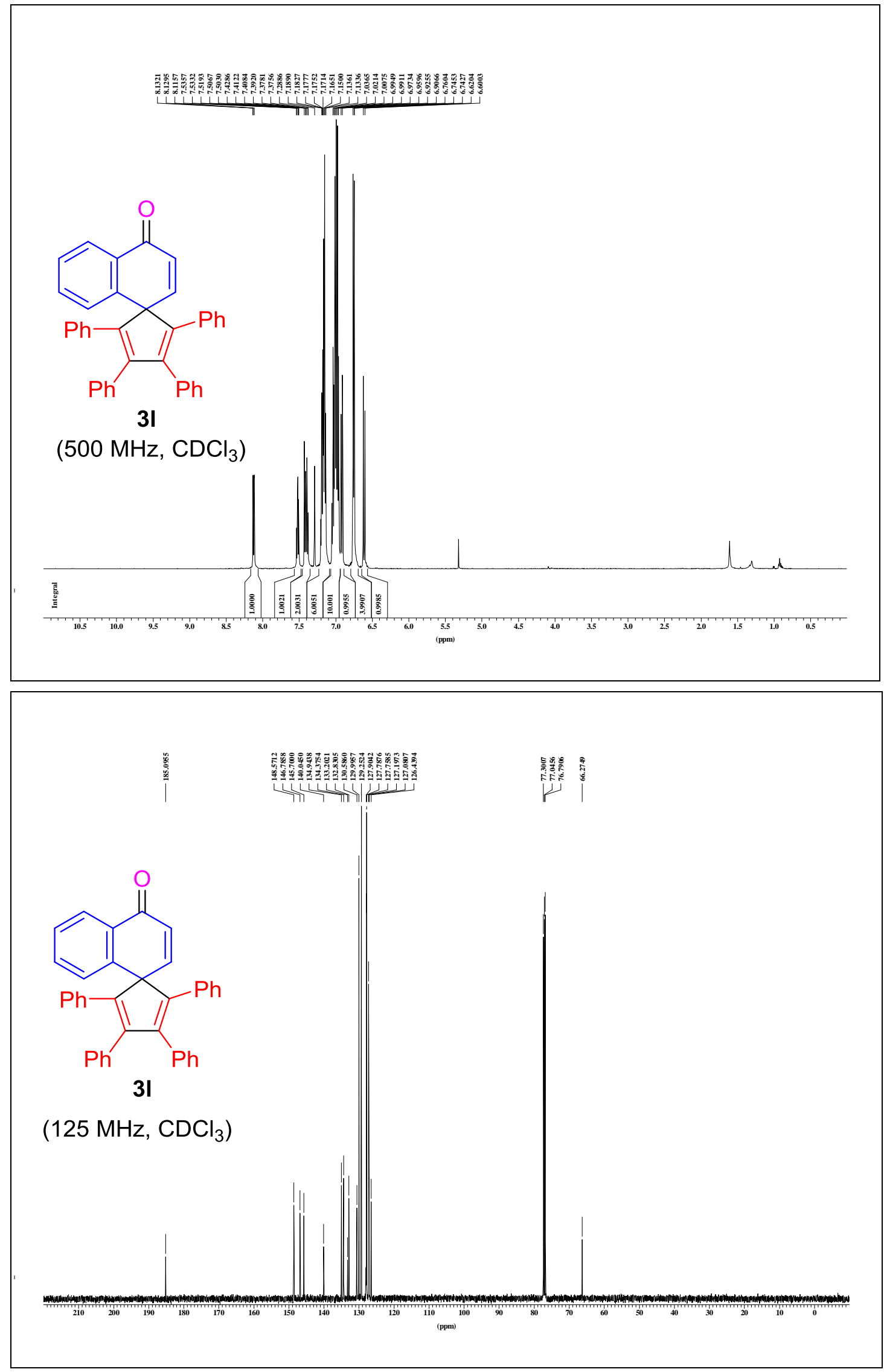



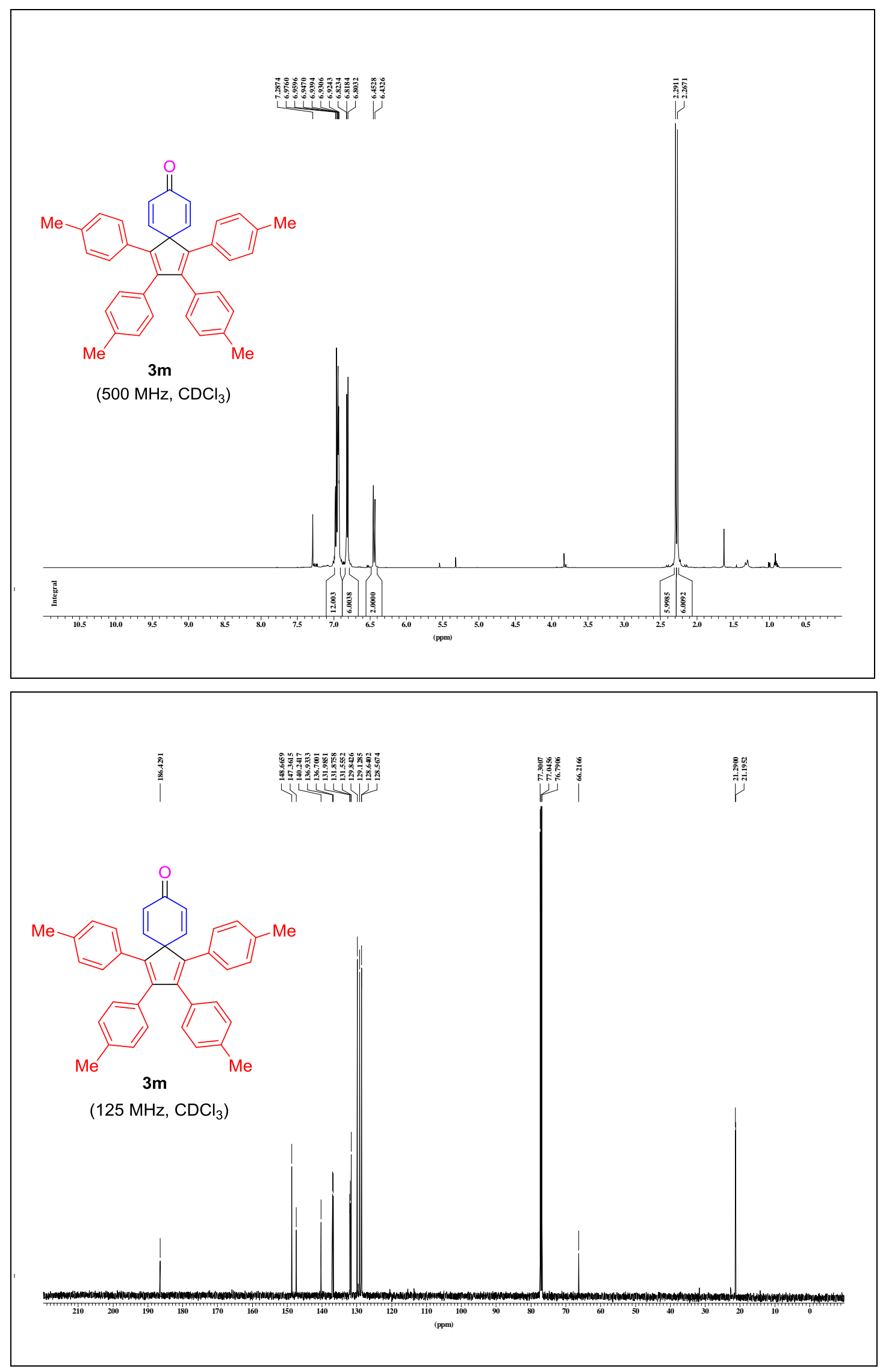

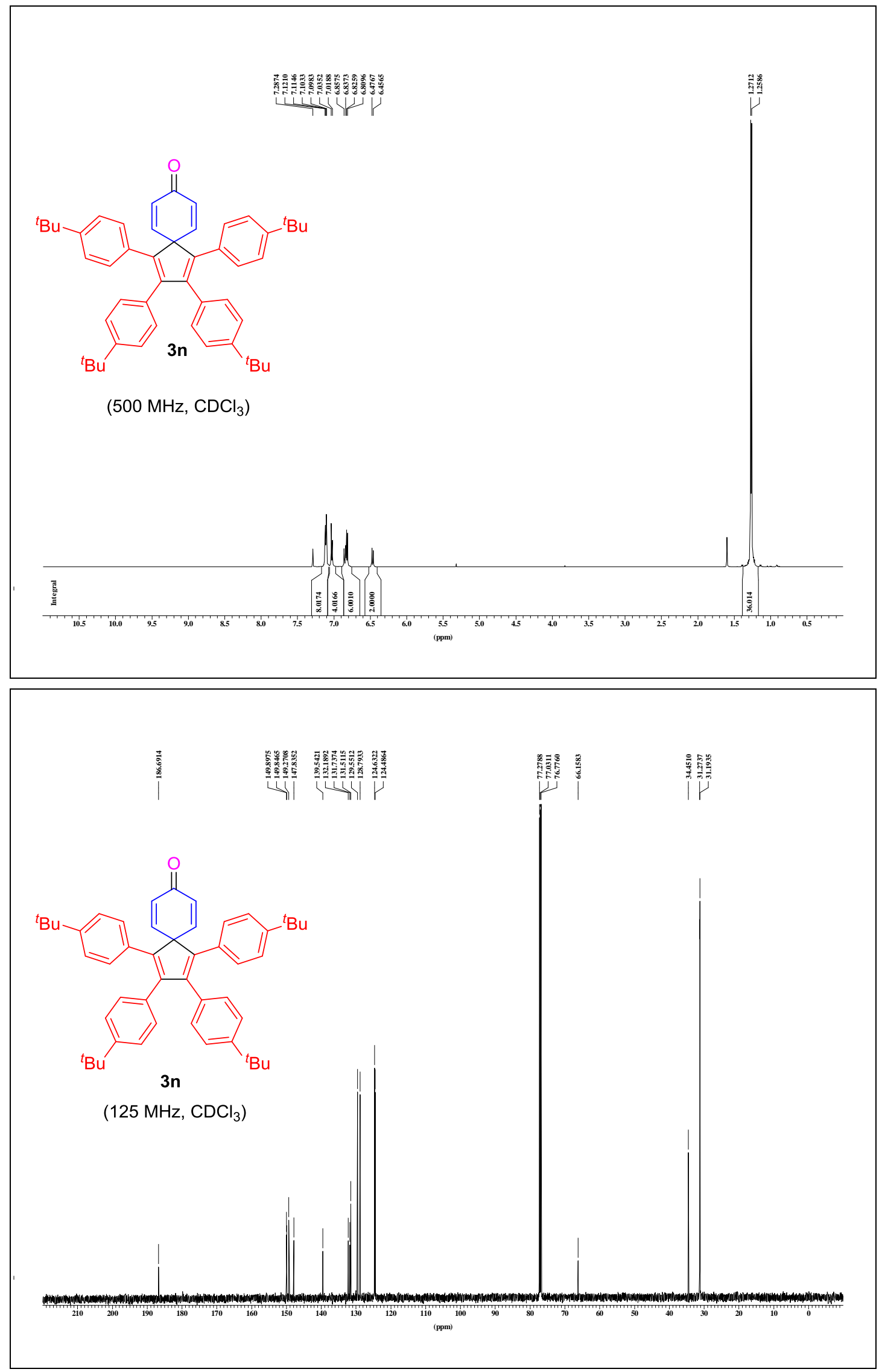

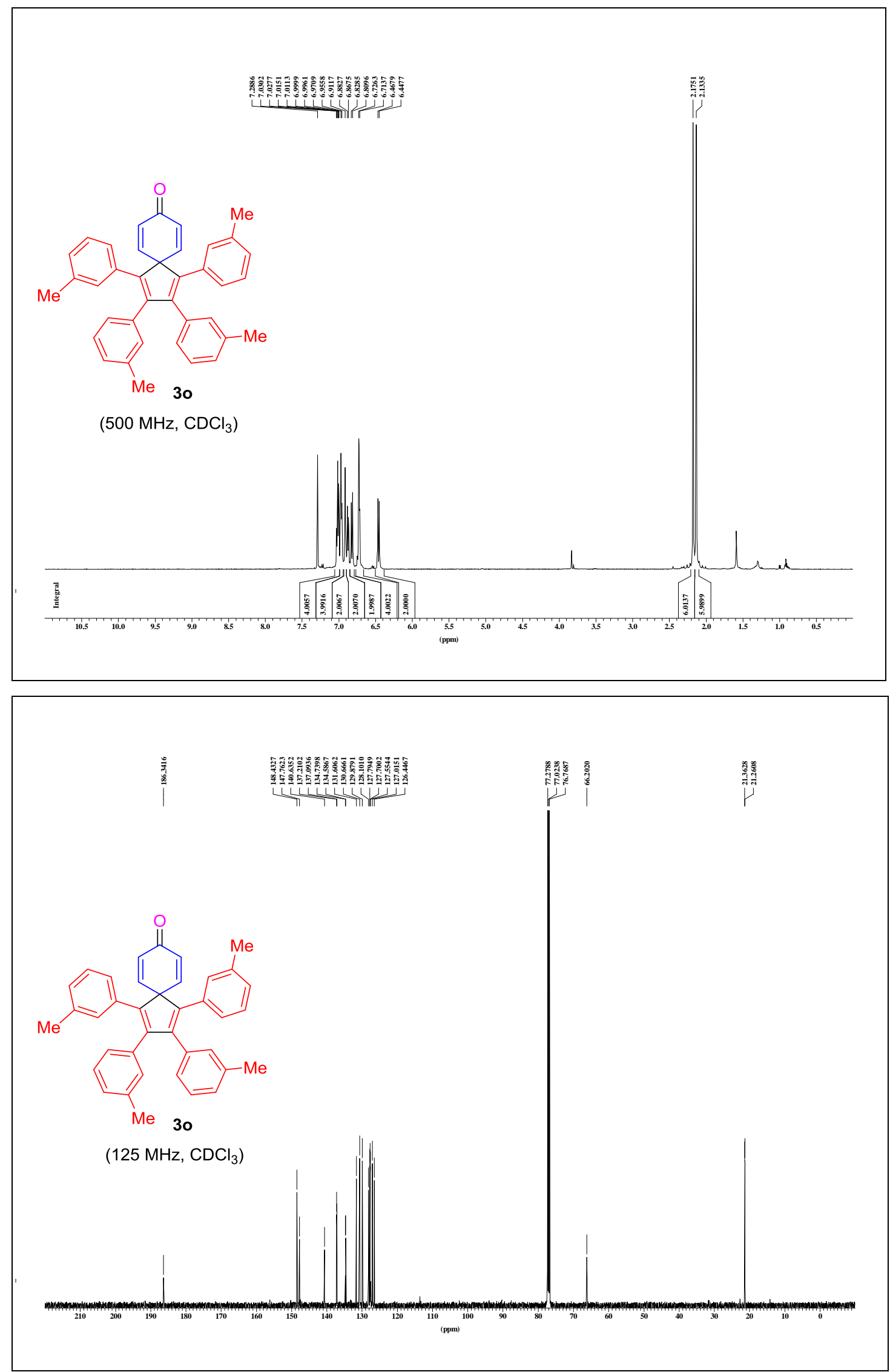

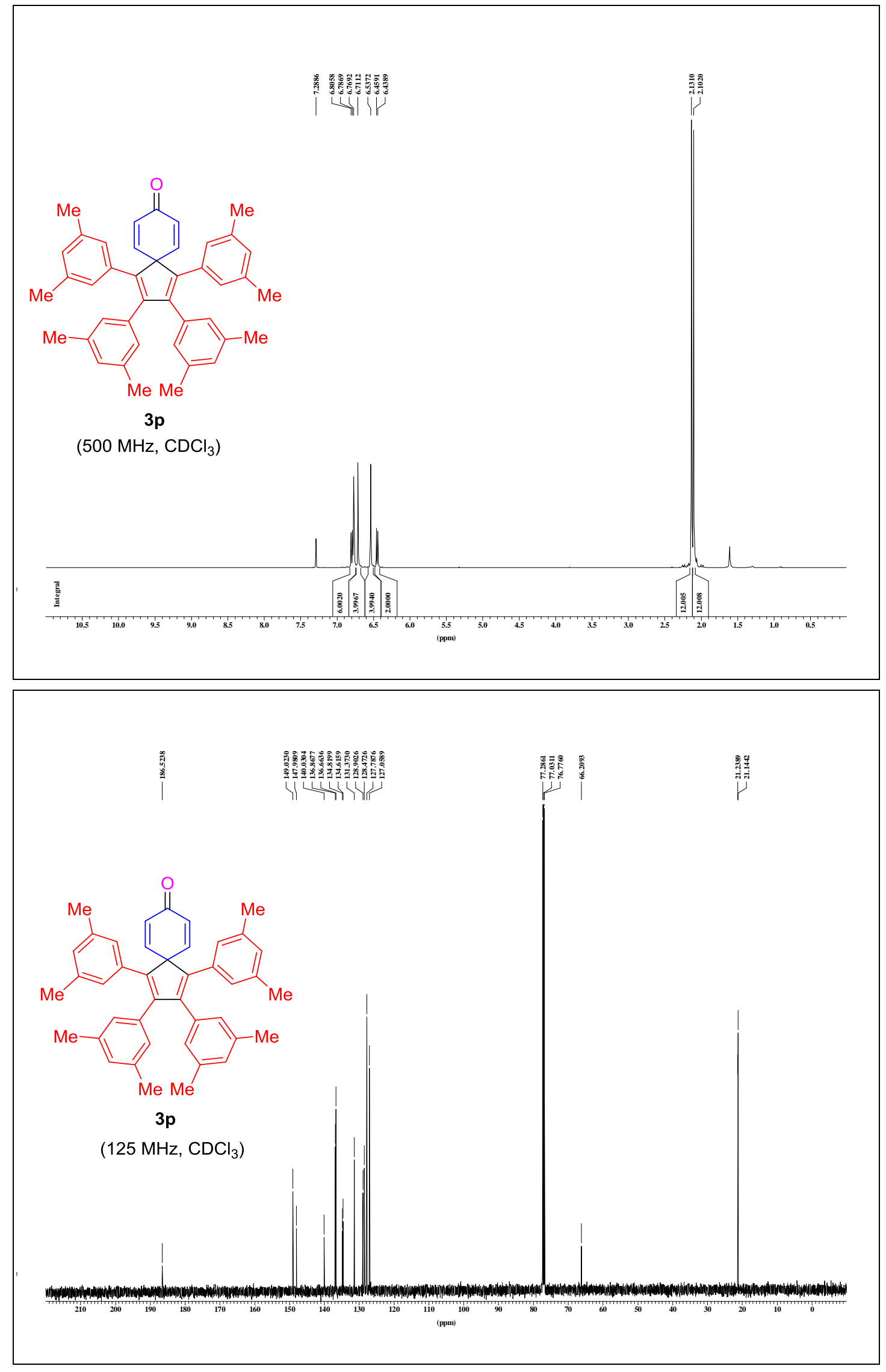

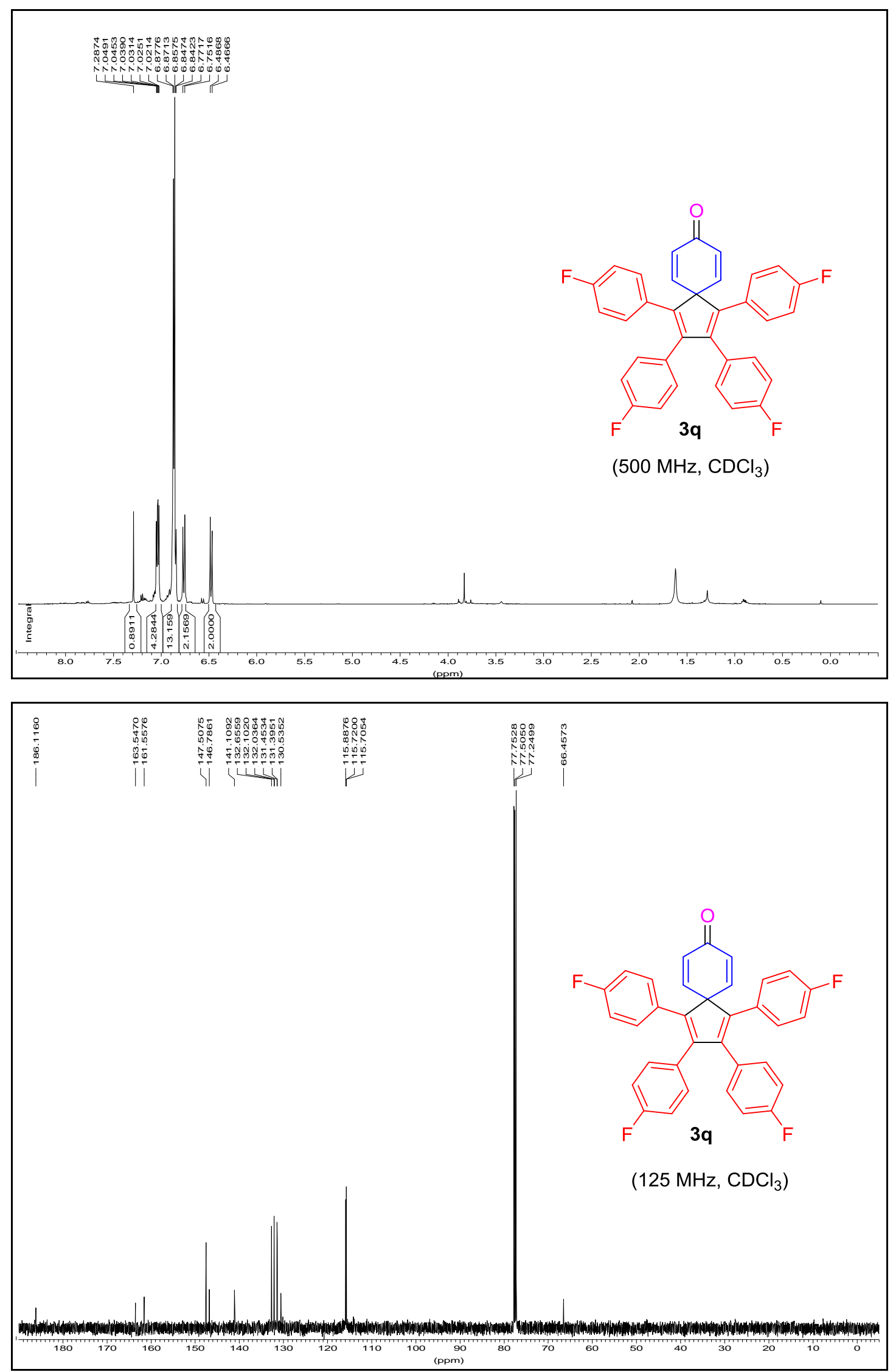

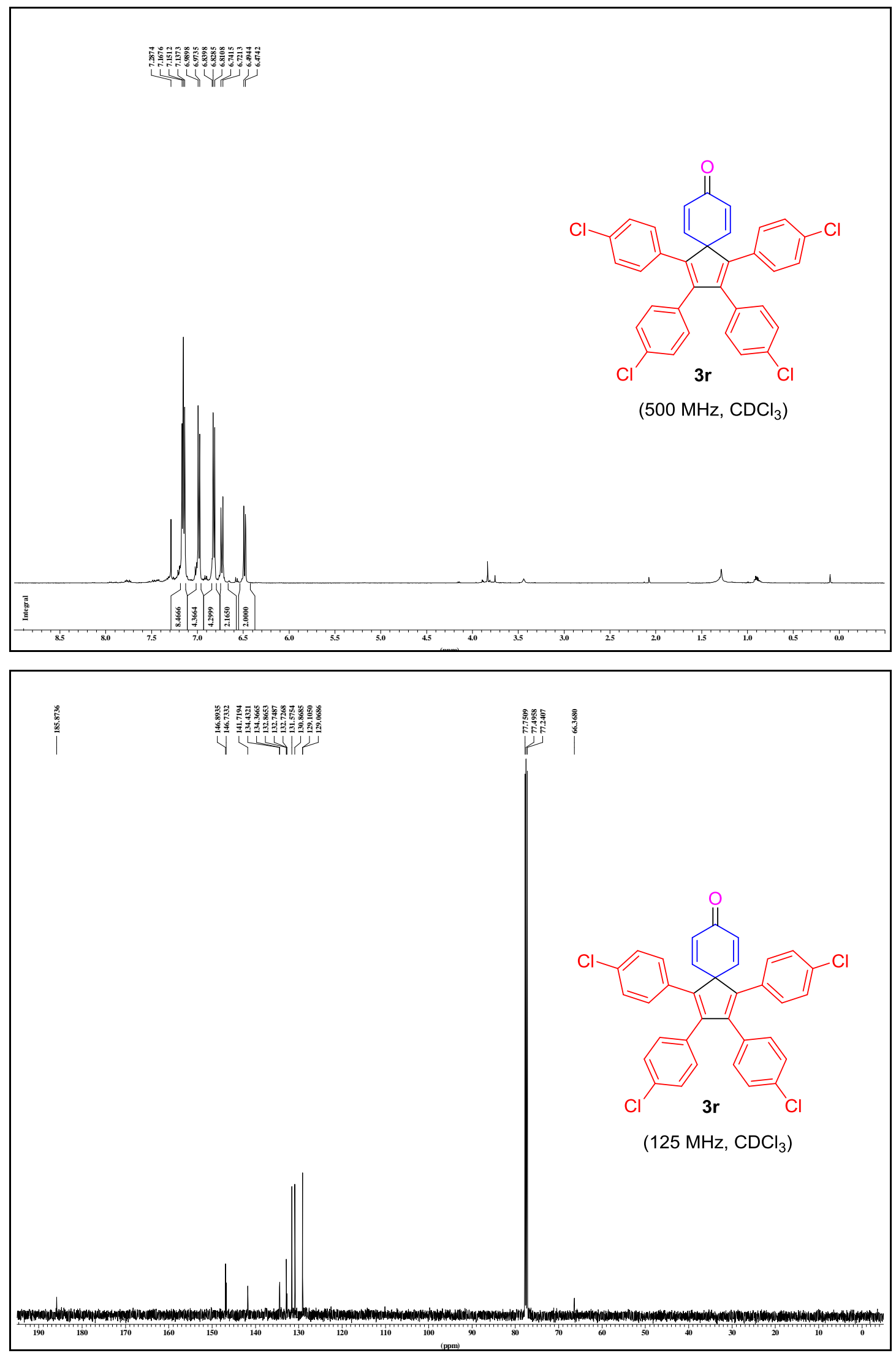


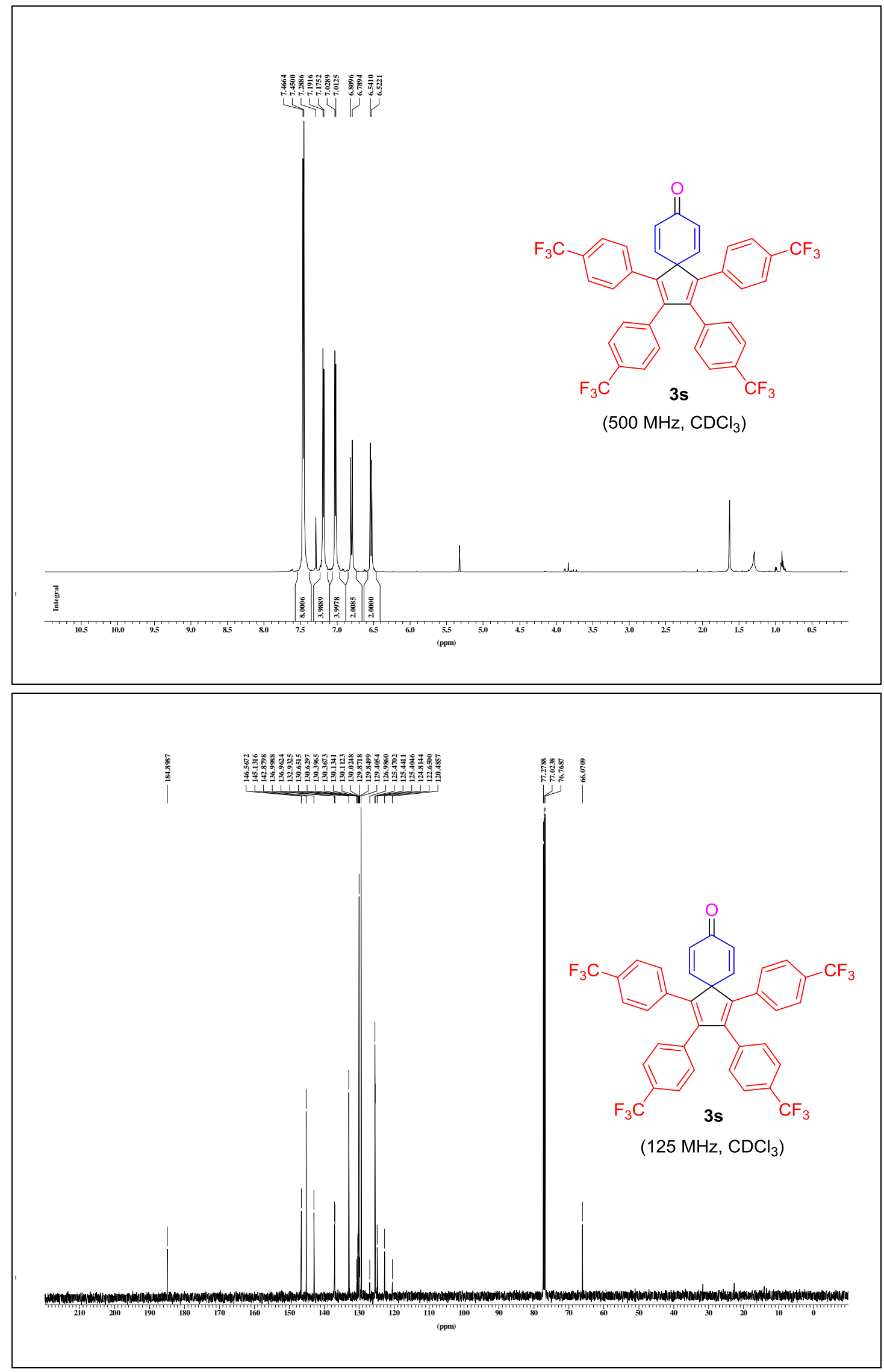



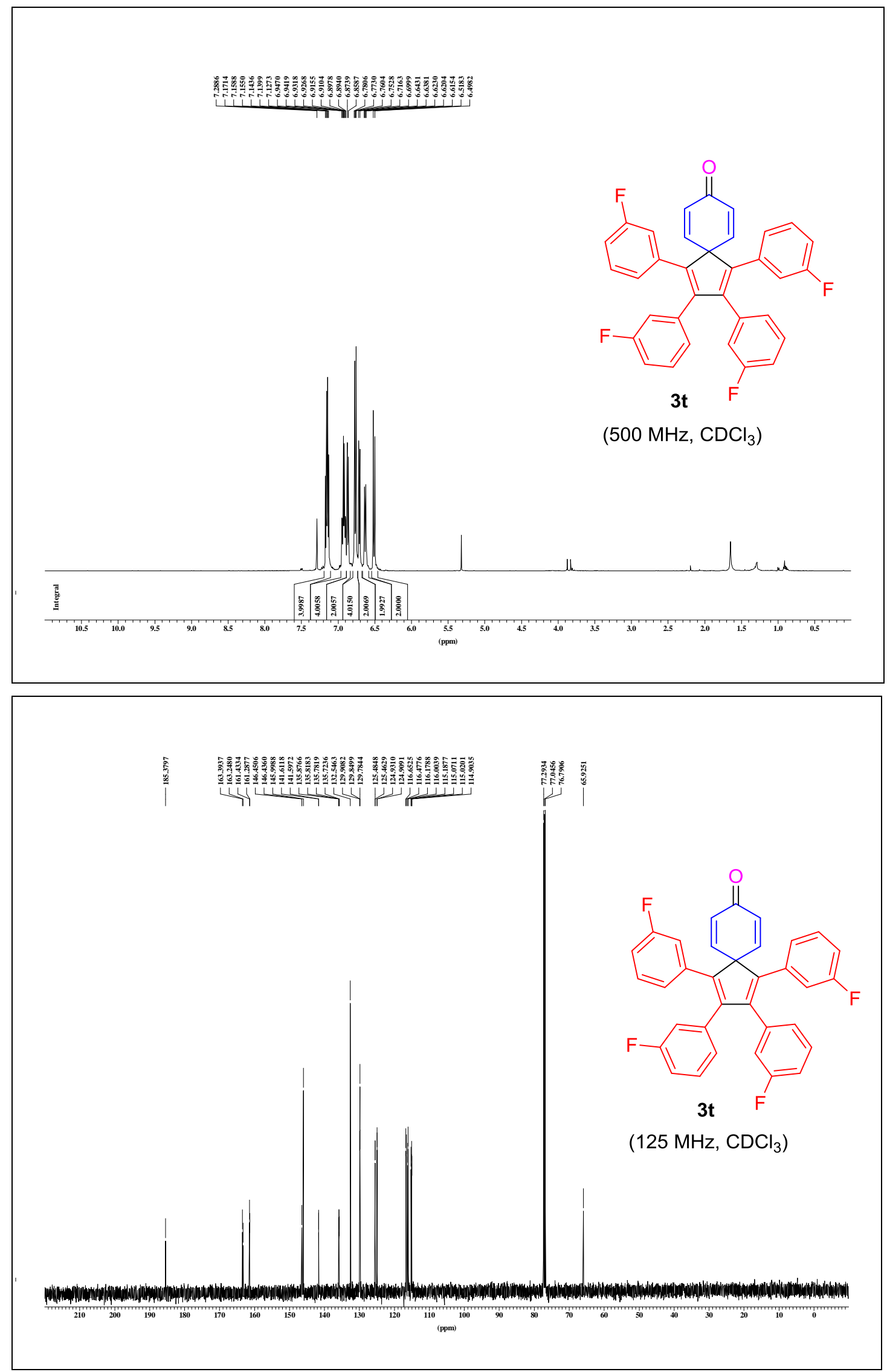


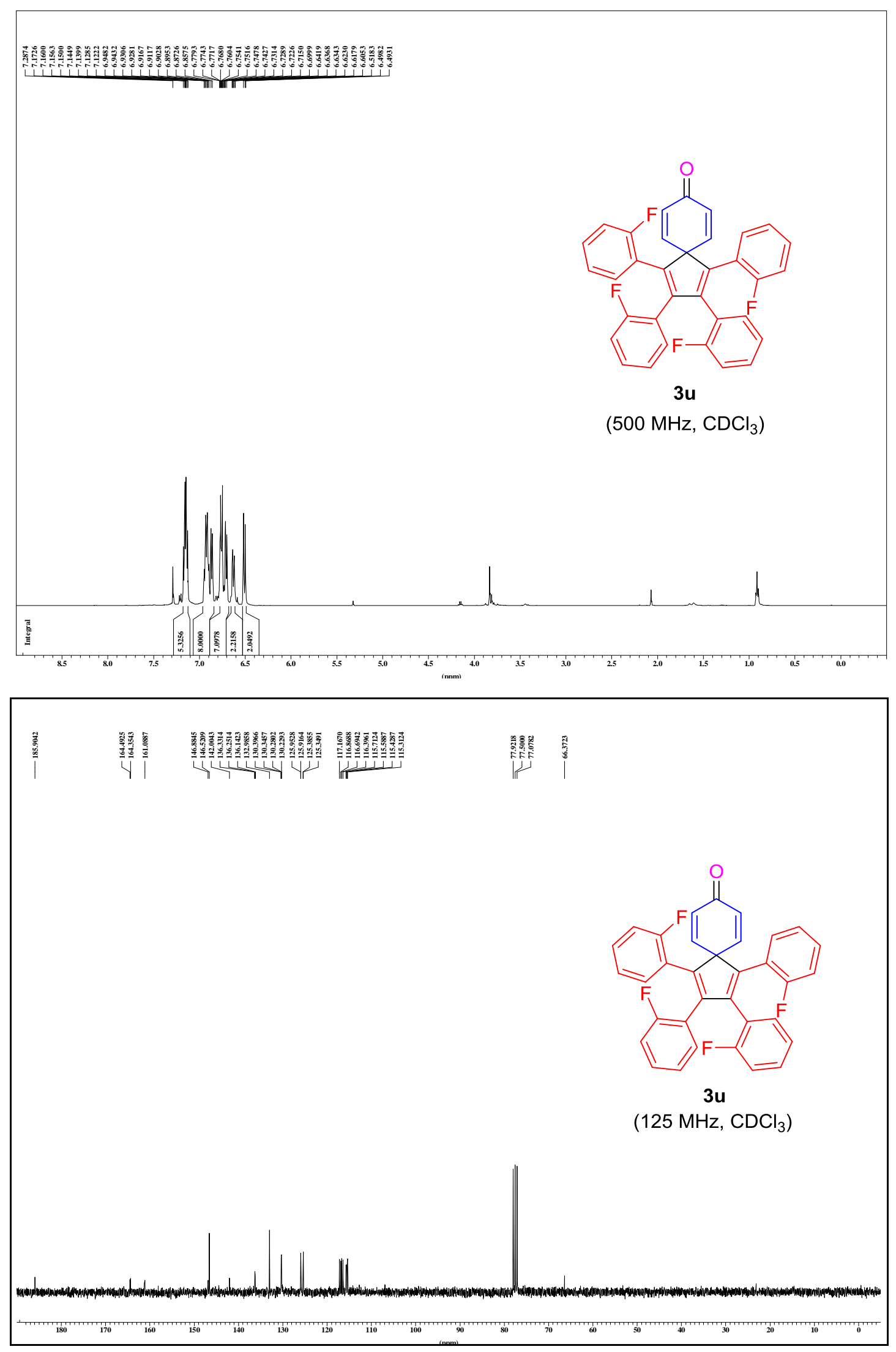




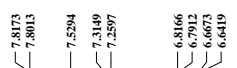

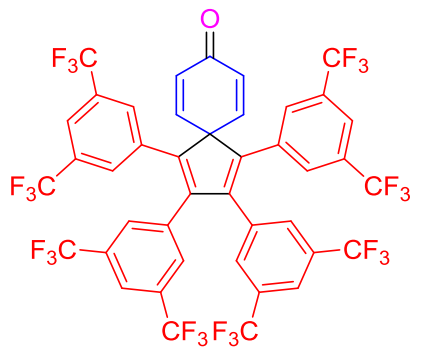

$3 v$

(500 MHz, $\mathrm{CDCl}_{3}$ )
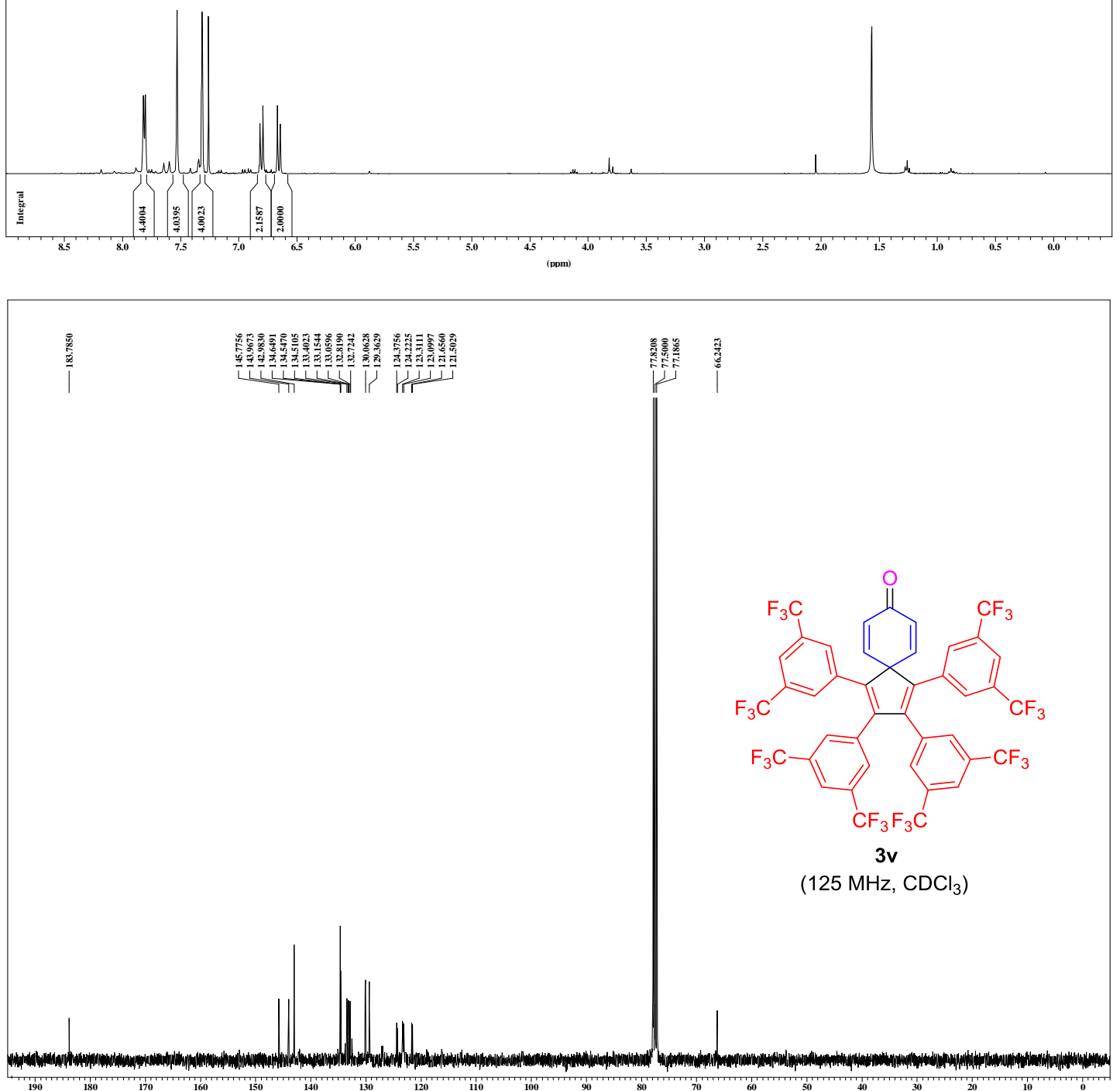

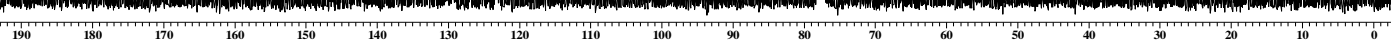



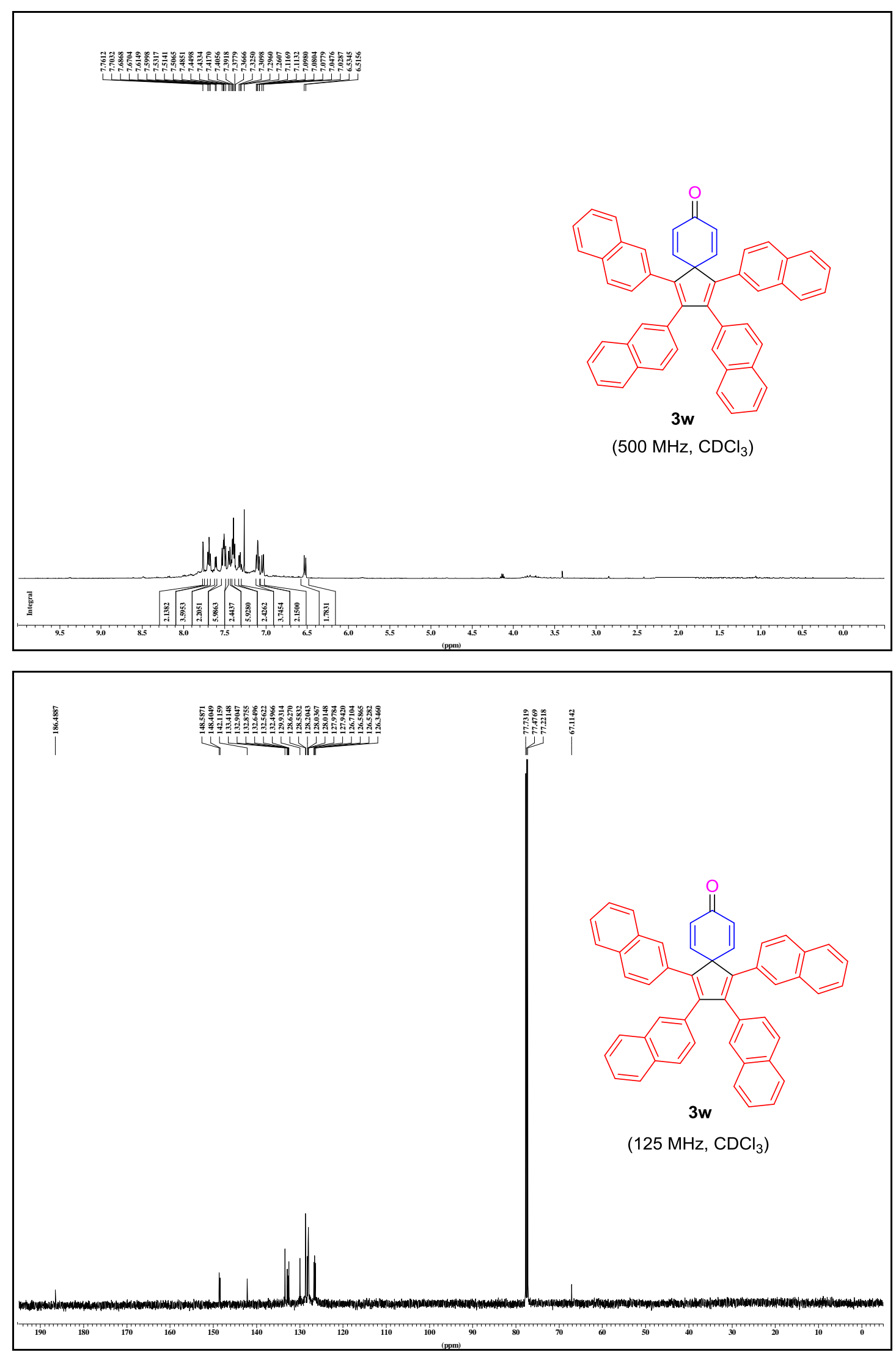

57 

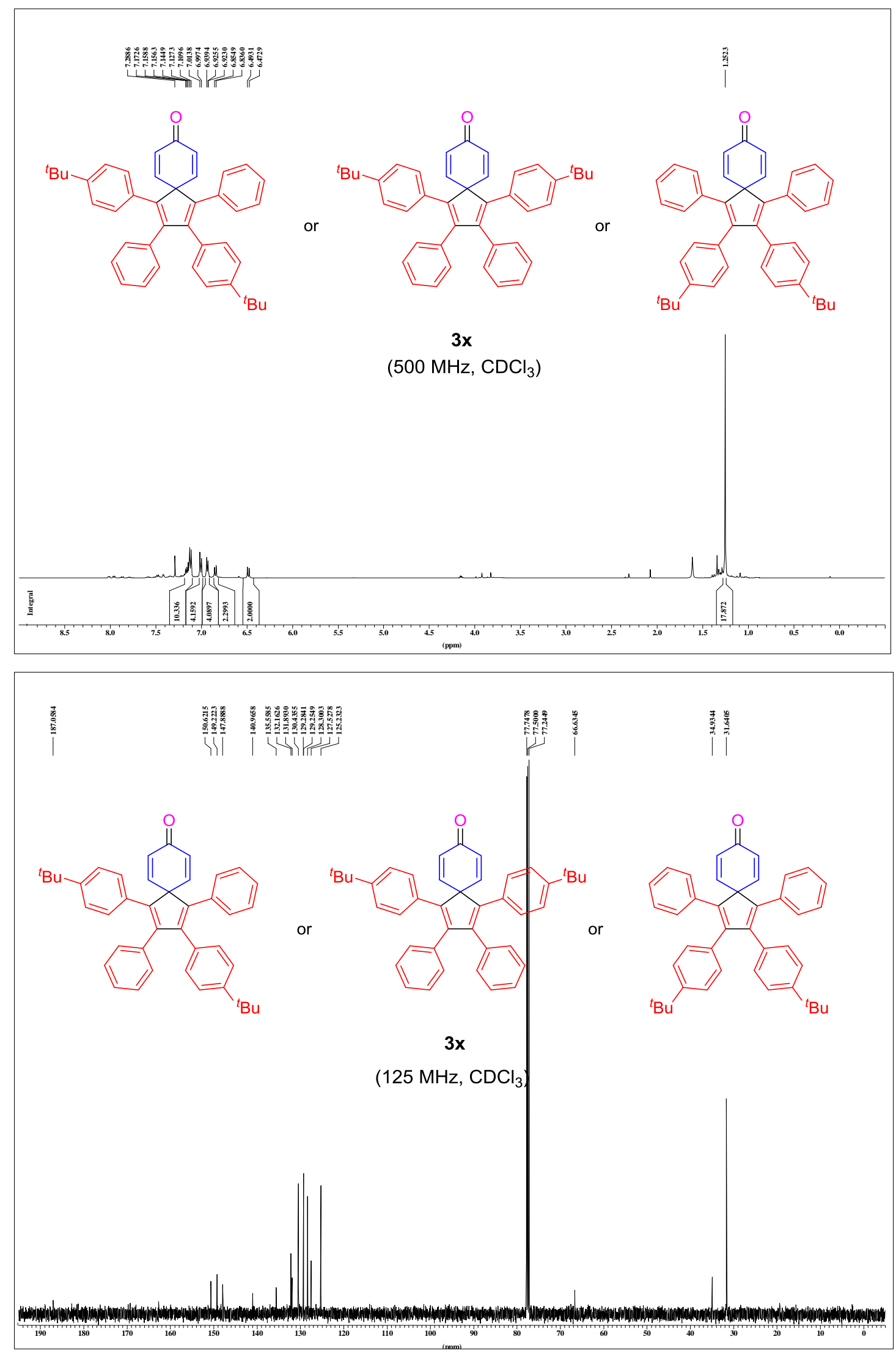

58 

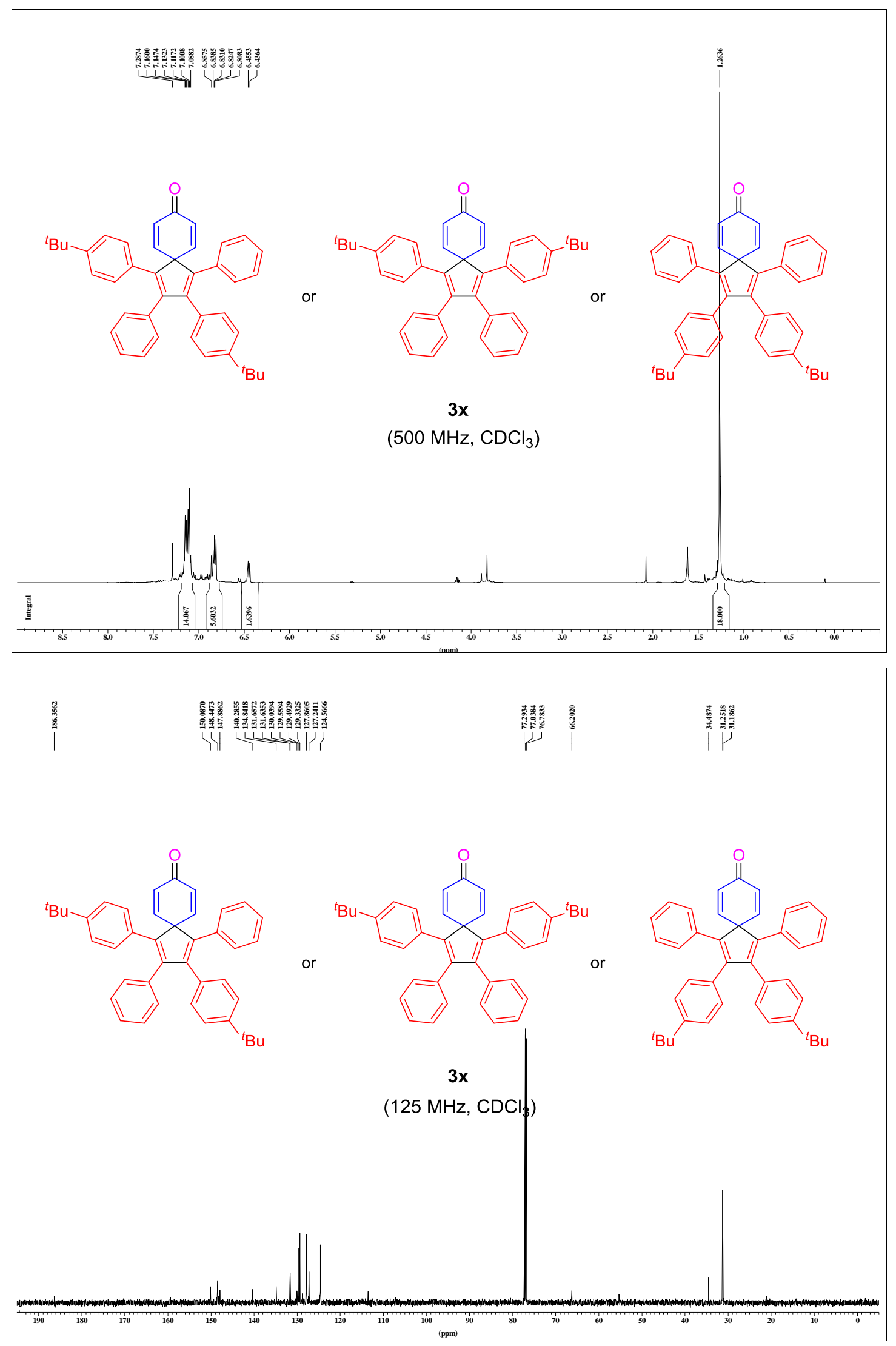

59 


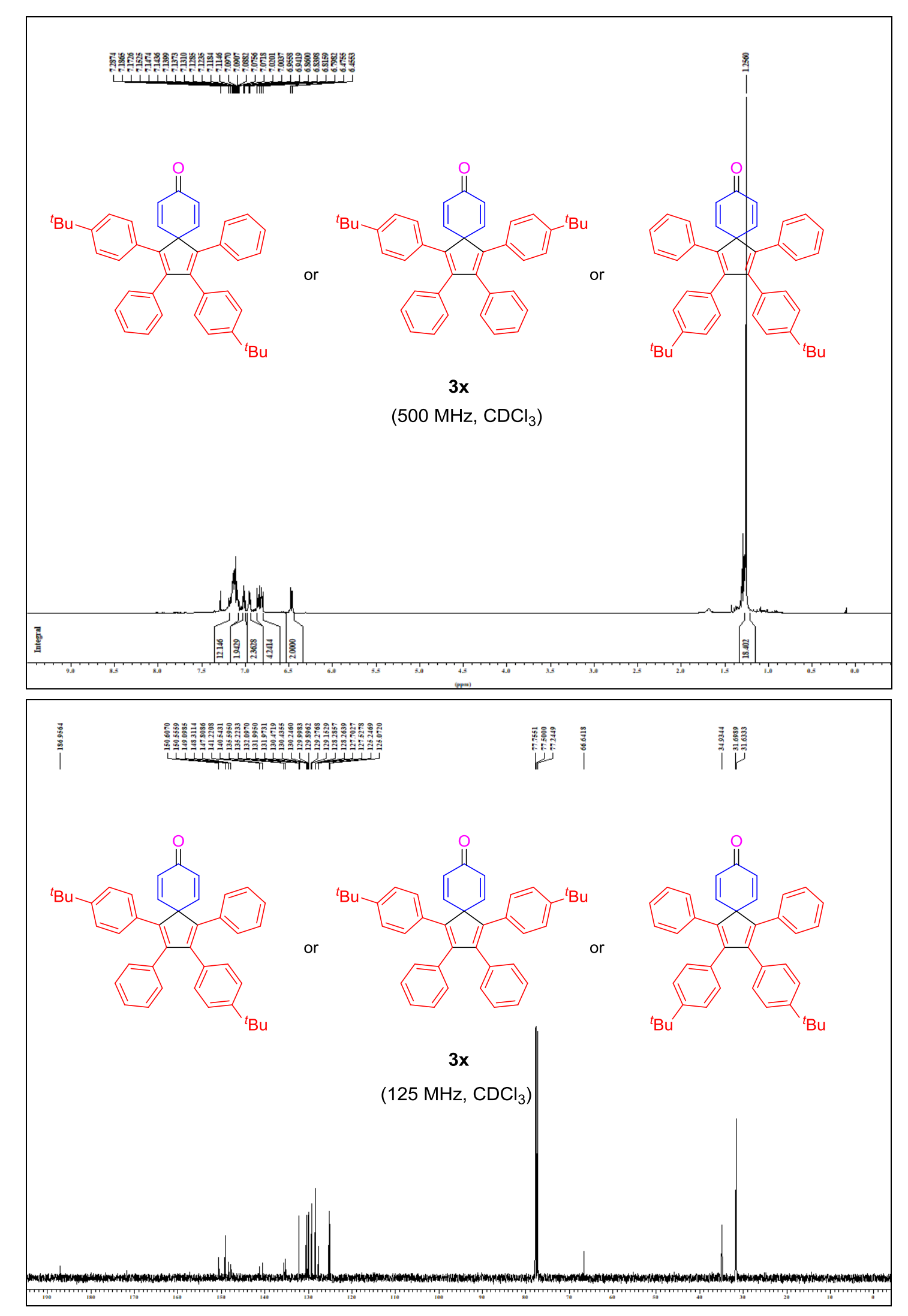




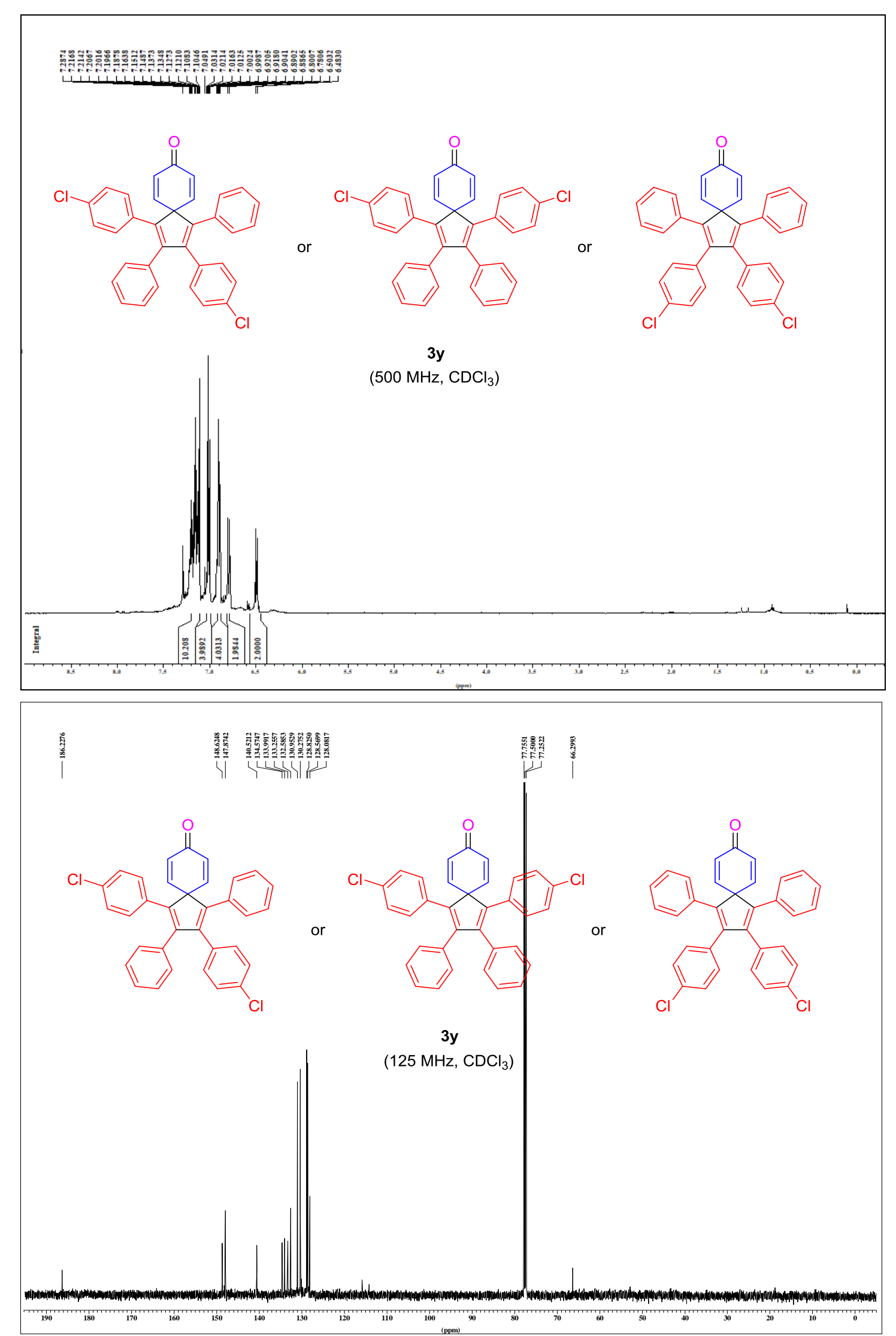




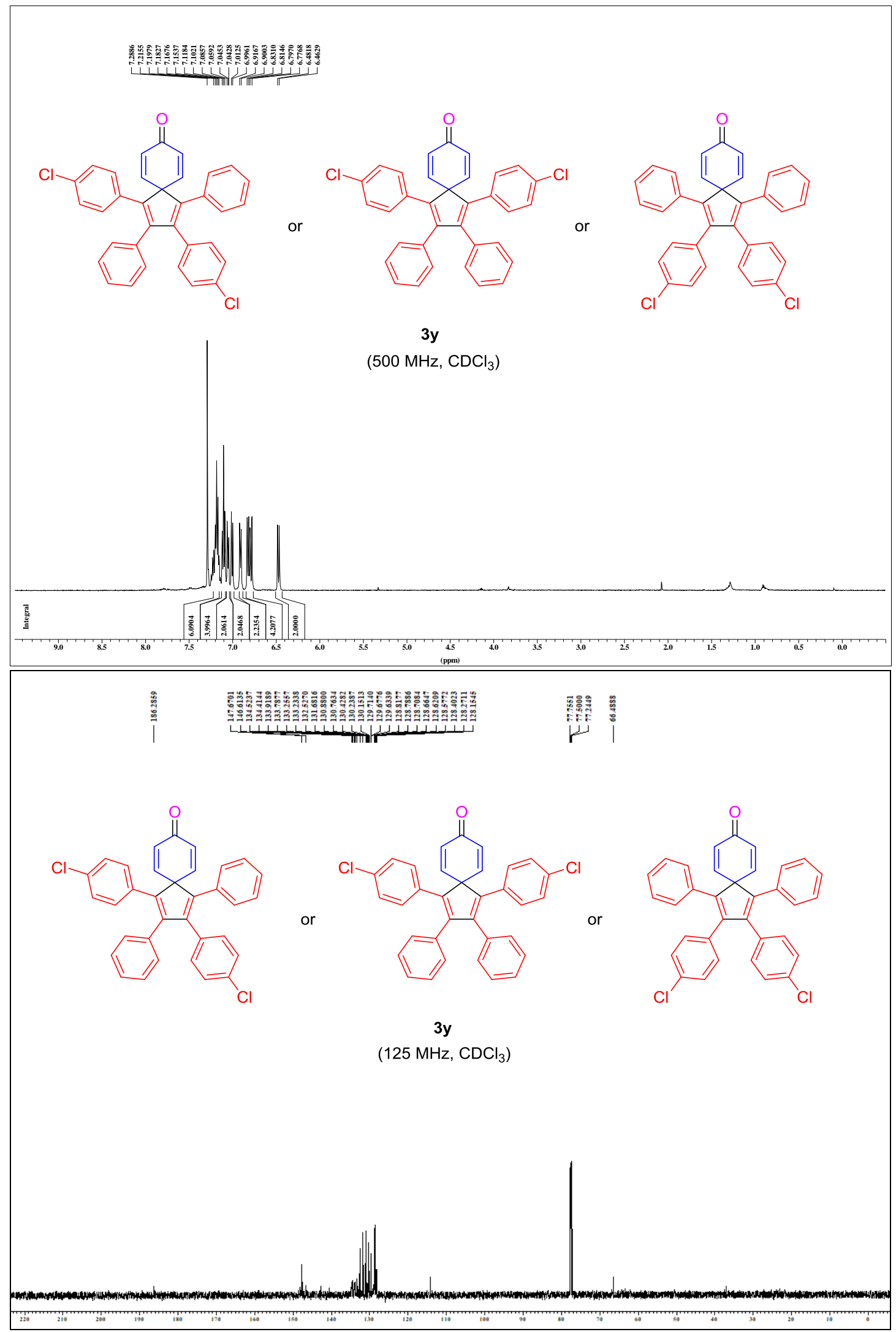




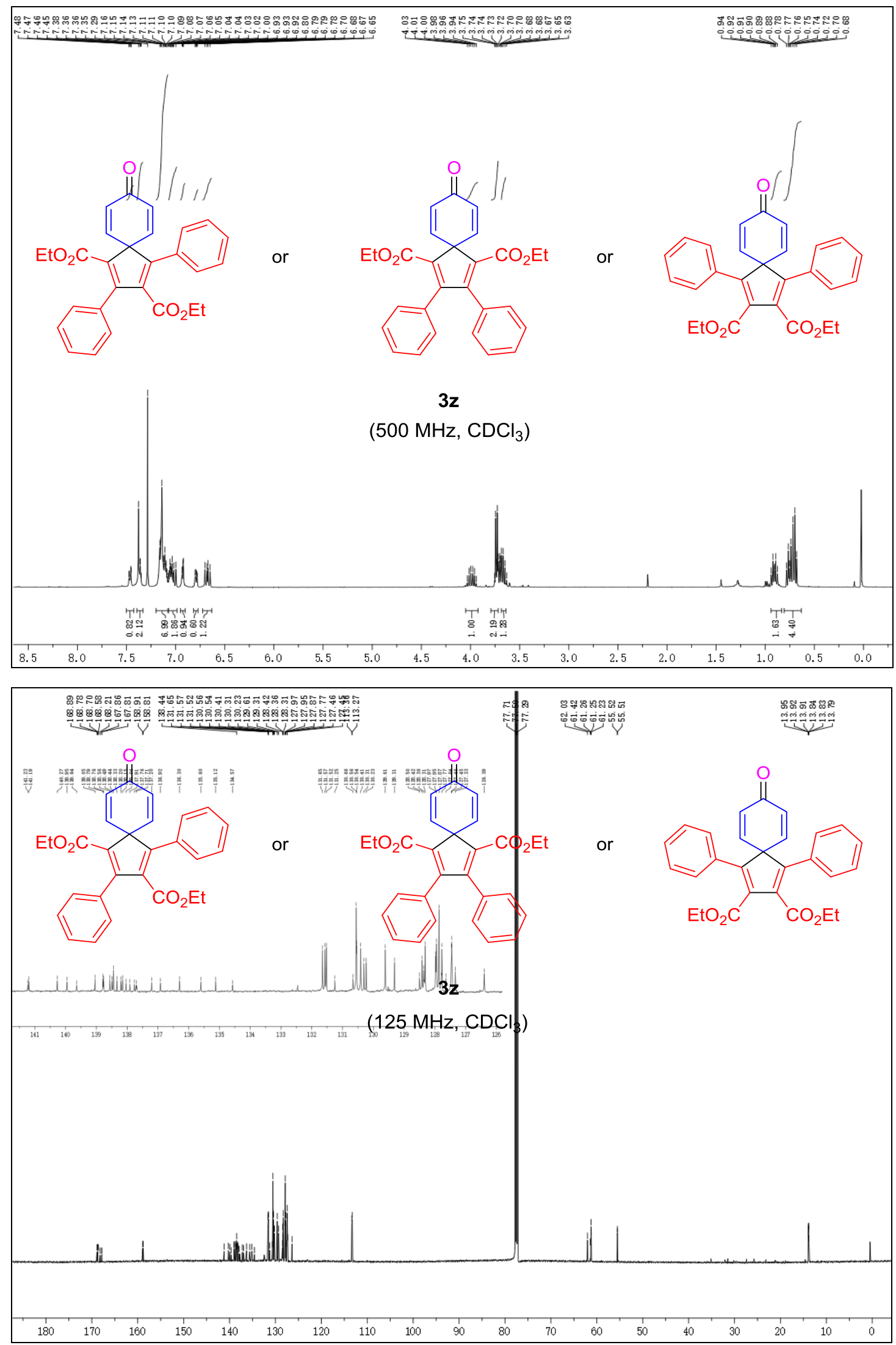



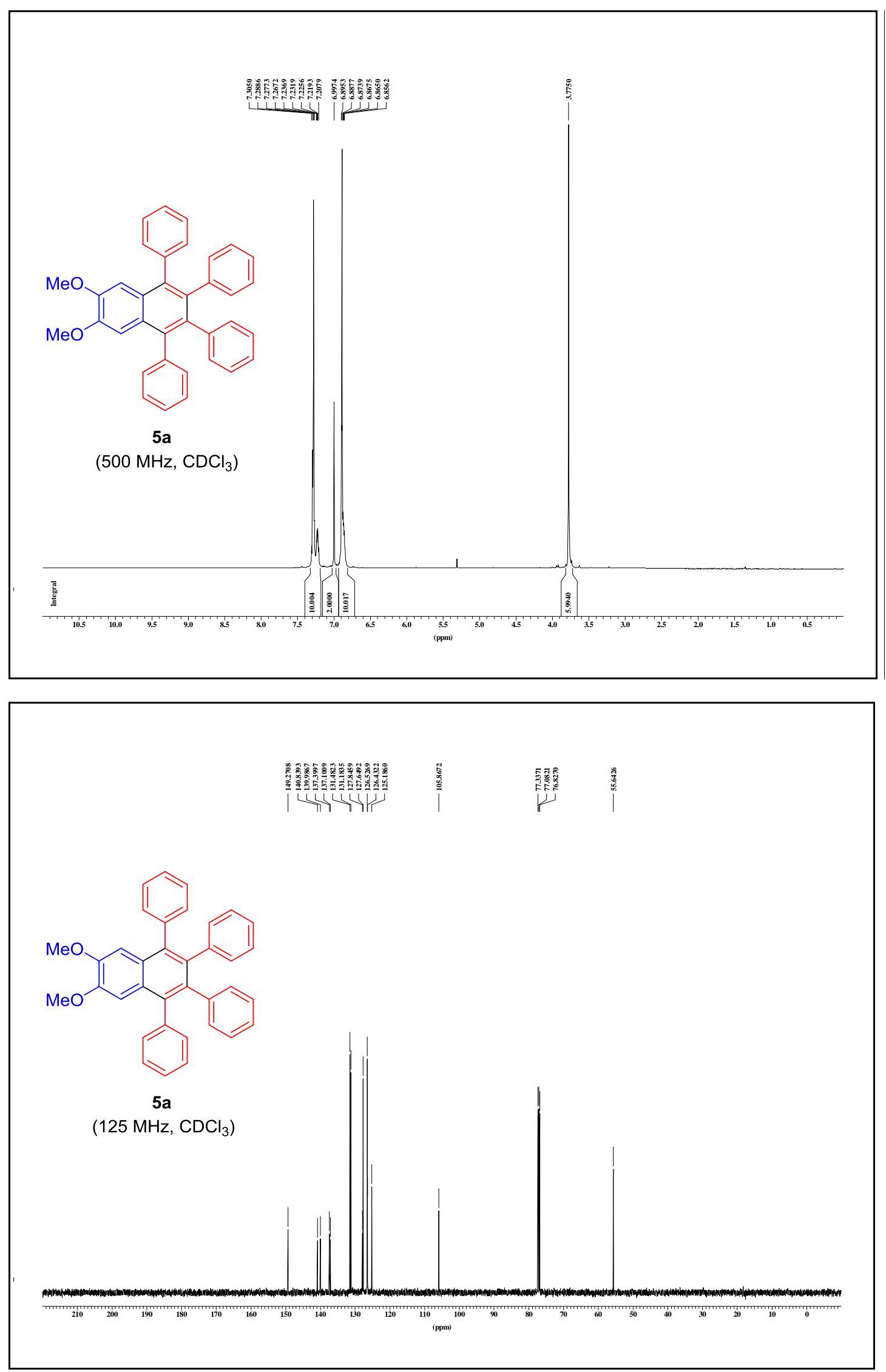

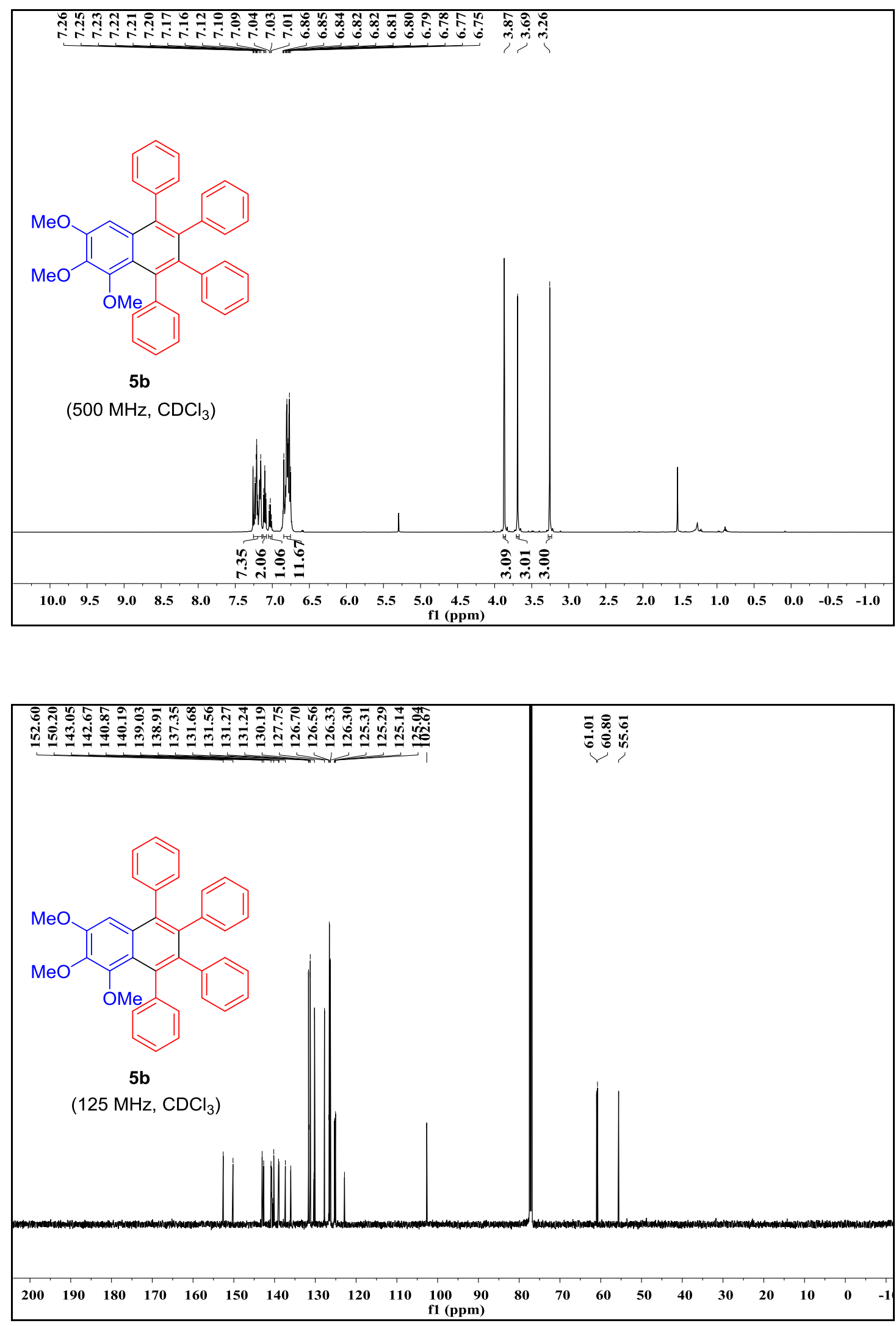

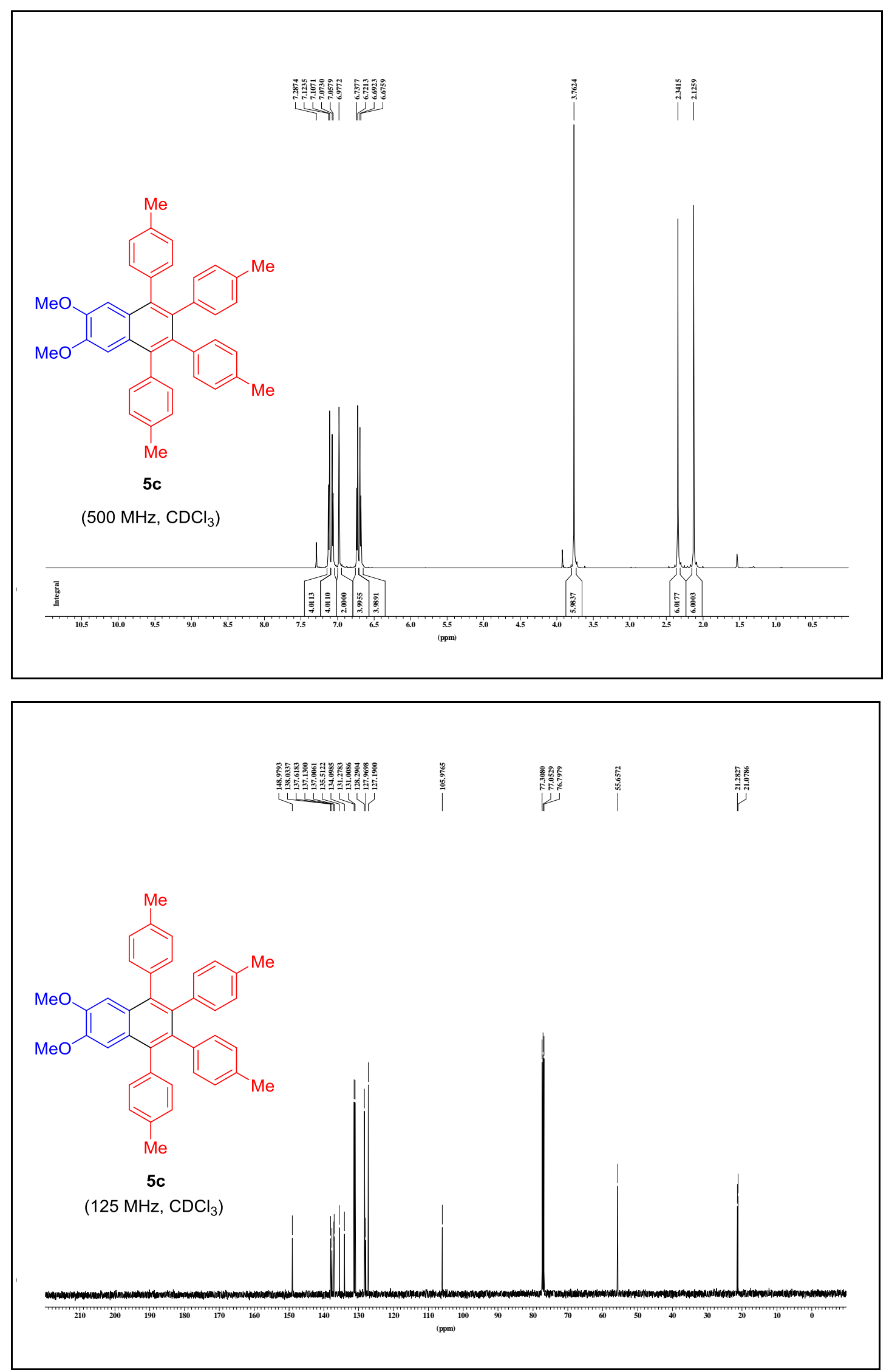

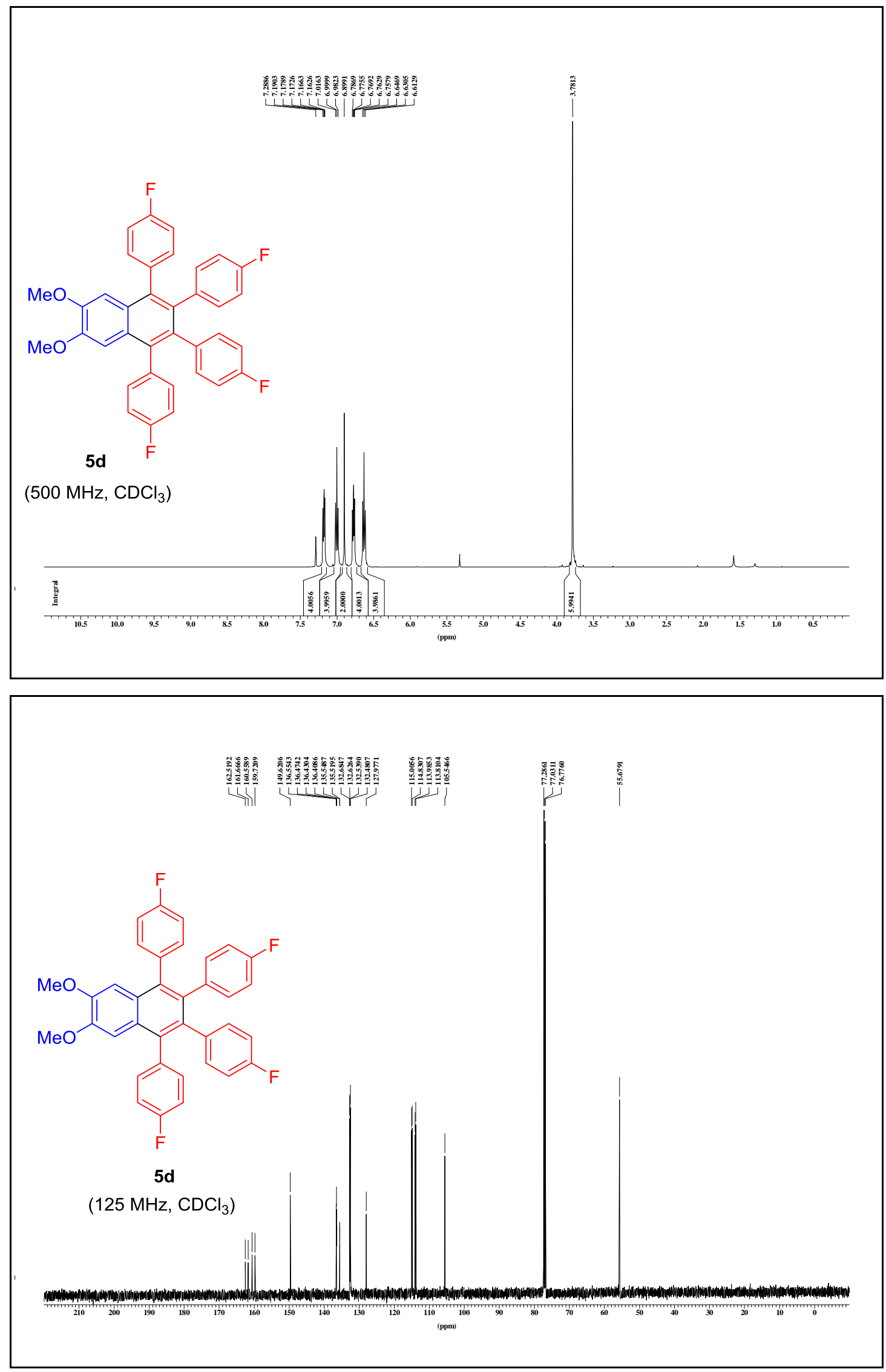

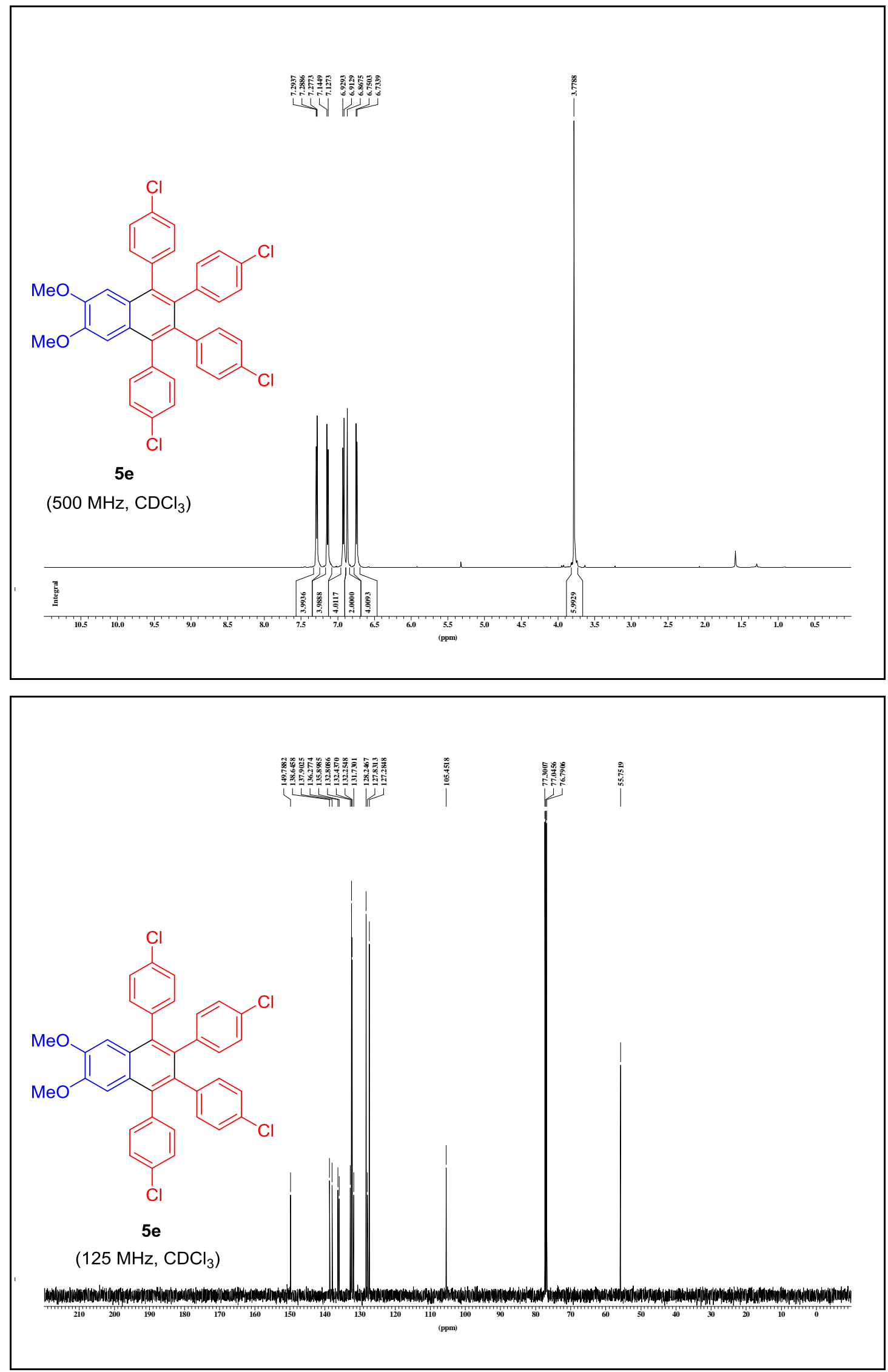

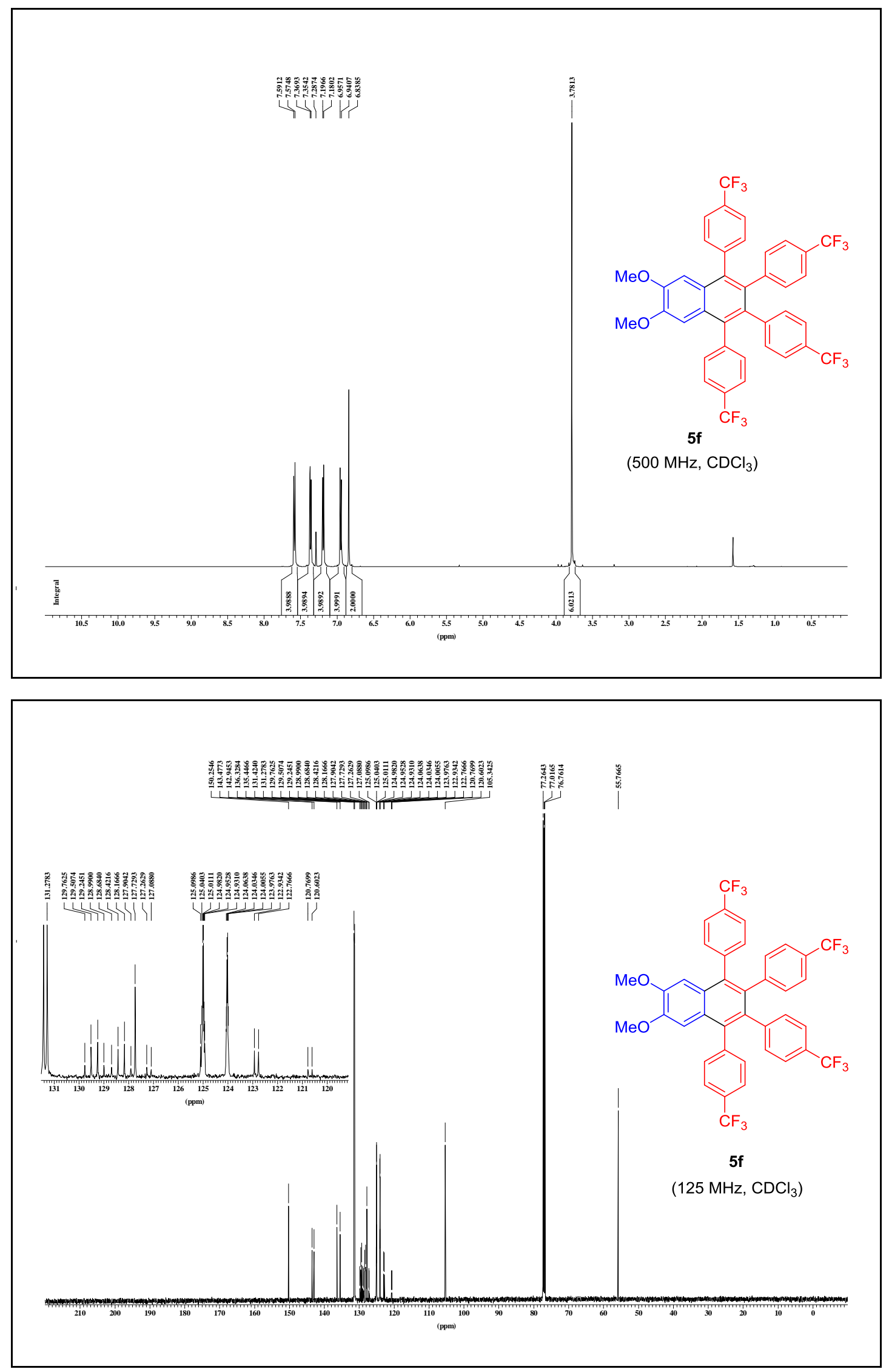

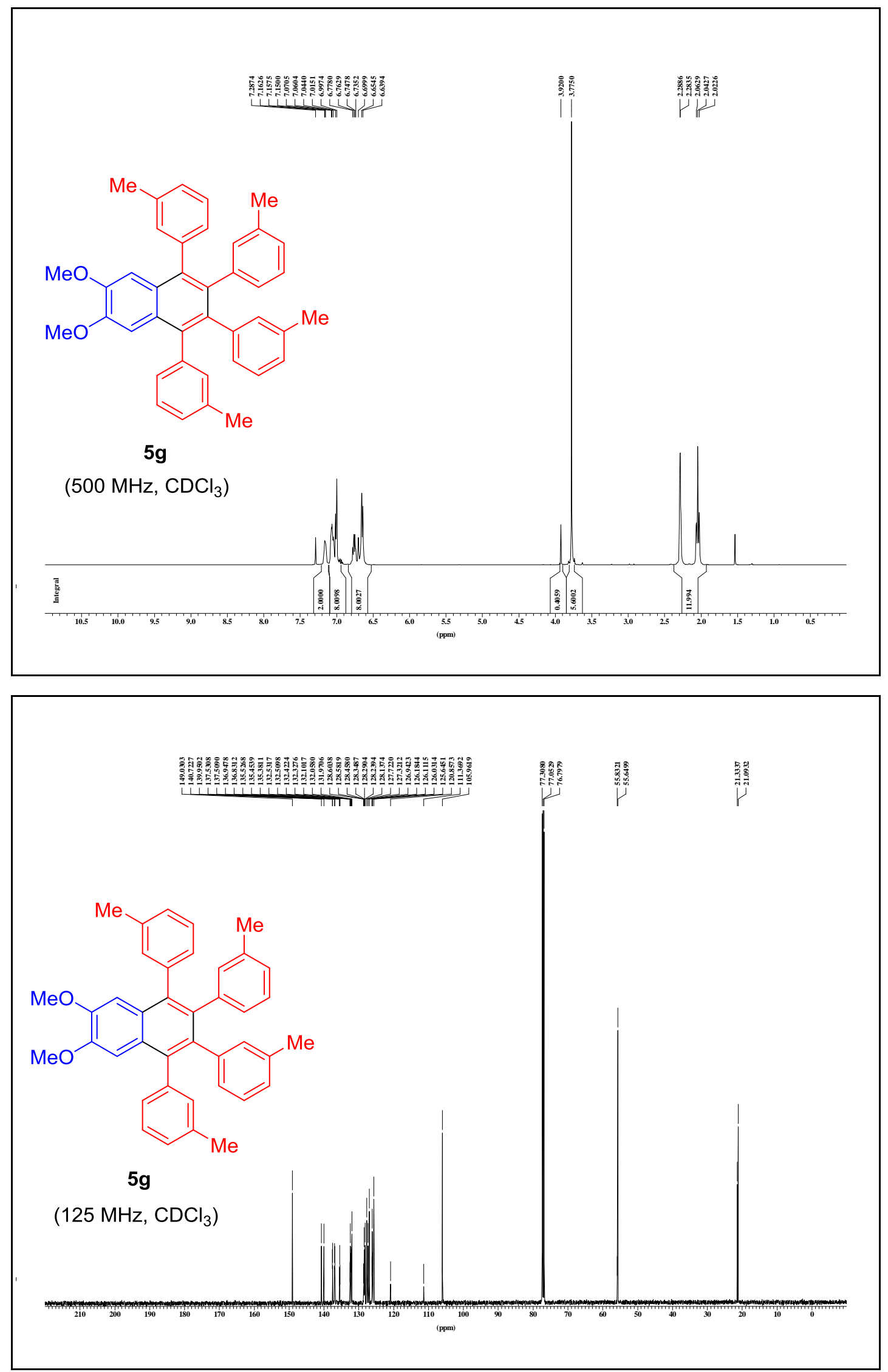

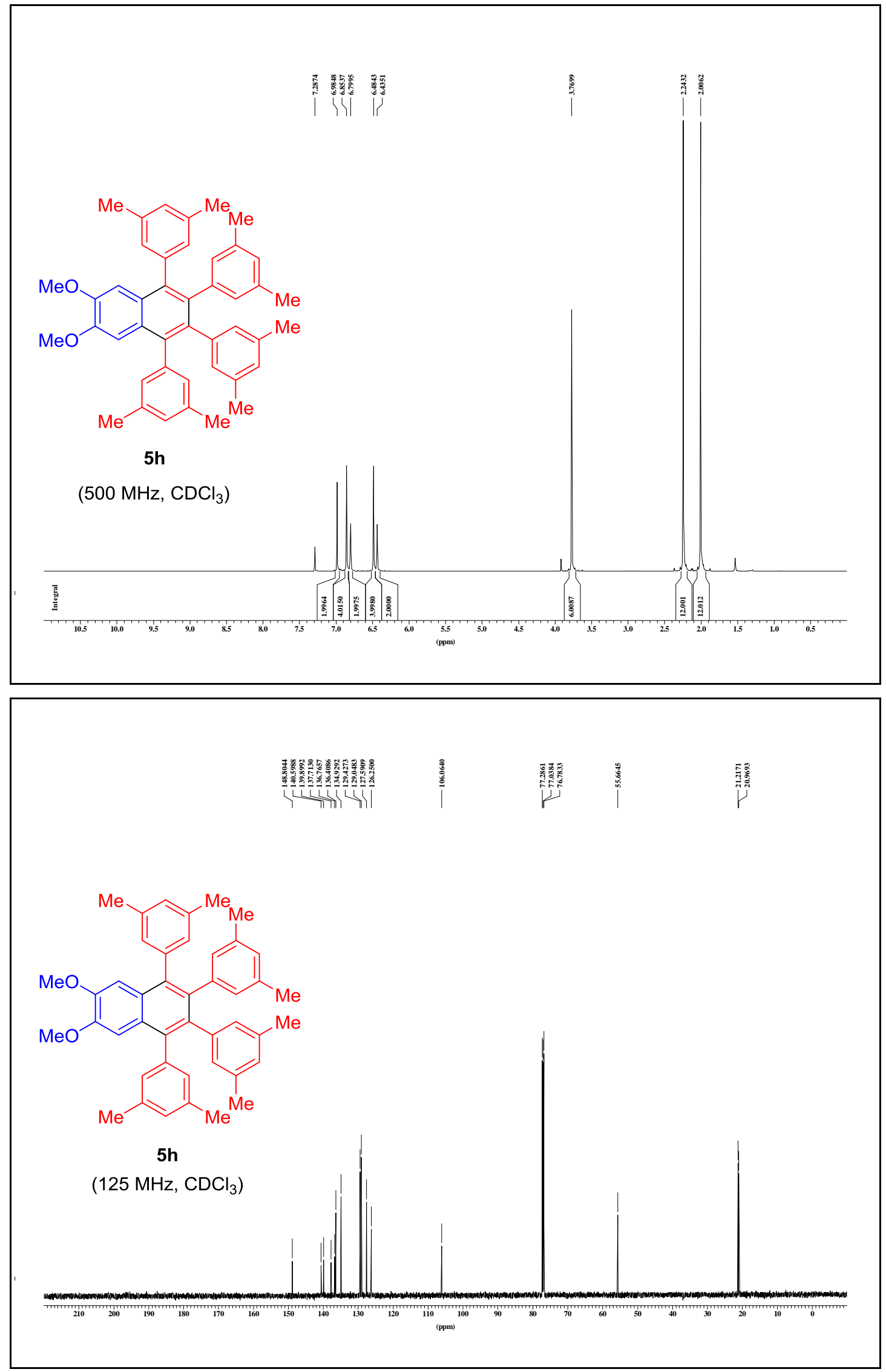

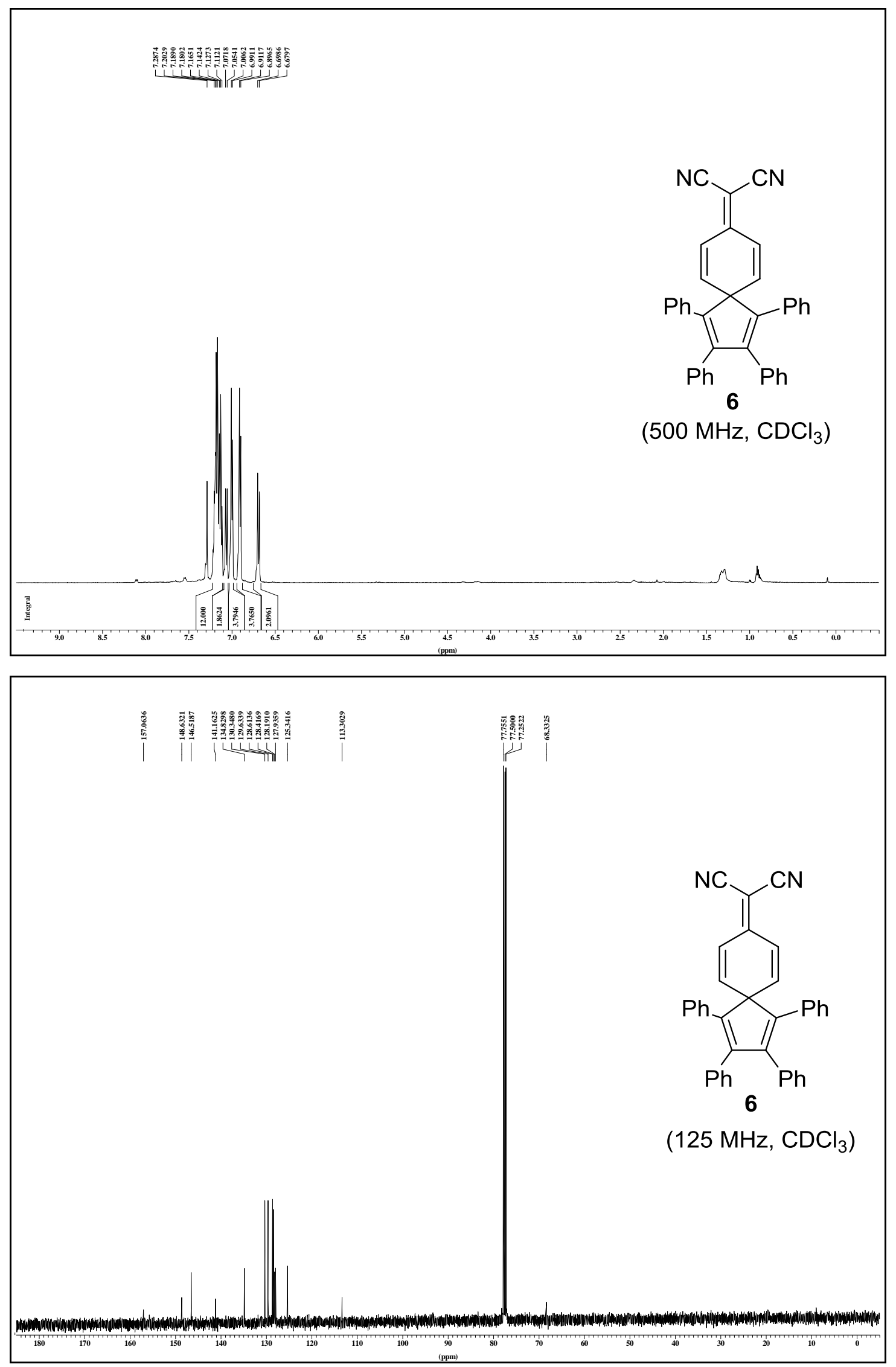

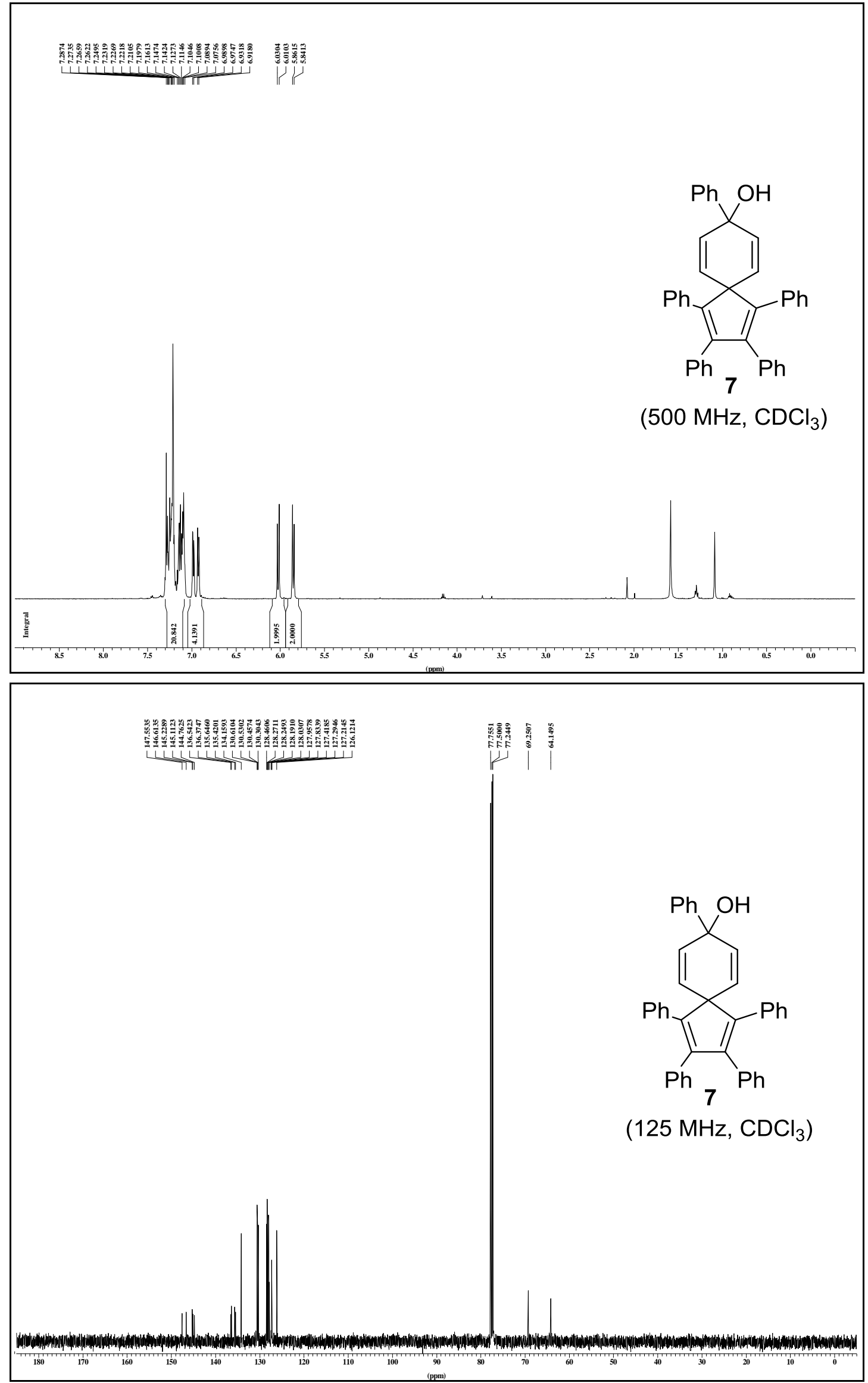